\title{
La flora della provincia di Pavia (Lombardia, Italia settentrionale). 1. L'Oltrepò Pavese
}

\author{
Nicola Maria Giuseppe Ardenghi ${ }^{1 *}$, Francesco Polani ${ }^{2}$
}

\begin{abstract}
Riassunto - Viene presentata ed analizzata la flora vascolare dell'Oltrepò Pavese, uno dei territori floristicamente più variegati e meno conosciuti dell'Italia nord-occidentale. L'opera costituisce il primo di tre contributi relativi alla flora della provincia di Pavia, per la quale mancava un lavoro di sintesi da oltre centocinquant'anni. I due elenchi floristici allegati si basano sui dati raccolti in campo dagli autori, integrati con le informazioni derivanti dallo studio critico della bibliografia esistente e dalla revisione dei campioni conservati soprattutto presso 1'Erbario Lombardo dell'Università di Pavia (PAV) e l'erbario del Museo di Storia Naturale di Milano (MSNM). Nell'area di studio risultano presenti 1.871 taxa (specie, sottospecie e ibridi), di cui 183 non più ritrovati dopo il 1980; a questi si aggiungono 245 entità di presenza dubbia e 161 segnalate per errore. Gli endemiti italiani ammontano a 16 mentre il contingente alloctono conta 321 entità (neofite e archeofite). Vengono qui segnalate 4 nuove neofite (Bidens triplinervia, Cucurbita melopepo subsp. melopepo, Morus kagayamae, Vitis berlandieri $\times V$. vinifera $)$ e una nuova archeofita $(\times$ Triticosecale $)$ per l'Italia; tra le 30 novità e le 16 conferme per la flora della Lombardia figurano 6 endemiti italiani (Adenostyles australis, Brachypodium genuense, Centaurea aplolepa s.1., Hieracium grovesianum s.1., Stipa etrusca, Tephroseris italica).
\end{abstract}

Parole chiave: Appennino Settentrionale, endemiti italiani, fitogeografia, flora alloctona, Pianura Padana.

Abstract - The Flora of the province of Pavia (Lombardy, northern Italy). 1. The Oltrepò Pavese.

The vascular flora of the Oltrepò Pavese, one of the most floristically diversified and neglected areas in north-western Italy, is here presented and discussed. This study represents the first of three contributions on the flora of the province of Pavia, which lacks a comprehensive floristic work since 1847. The two attached checklists are based on field data collected by the authors, integrated with information acquired from the critical analysis of the available bibliographical sources and the revision of the specimens mainly preserved at the Erbario Lombardo of the University of Pavia (PAV) and the herbarium of the Museo di Storia Naturale di Milano (MSNM). The flora of the Oltrepò Pavese includes 1.871 taxa (species, subspecies and hybrids), among them 183

\footnotetext{
${ }^{1}$ Dipartimento di Scienze della Terra e dell'Ambiente, Università degli Studi di Pavia, Via Sant'Epifanio 14, 27100 Pavia, Italia

${ }^{2}$ Via Filippo Turati 3, 27049 Stradella (PV), Italia

* Corresponding author: sahfen@hotmail.com

(C) 2016 Nicola Maria Giuseppe Ardenghi, Francesco Polani
}

Received: 27 January 2016

Accepted for publication: 16 September 2016 no longer recorded after 1980; 245 doubtfully present taxa and 161 taxa erroneously recorded in the past are additionally reported. Species and subspecies endemic to Italy amount to 16 while the alien taxa to 321 (neophytes and archaeophytes). 4 new neophytes (Bidens triplinervia, Cucurbita melopepo subsp. melopepo, Morus kagayamae, Vitis berlandieri $\times V$. vinifera $)$ and one new archaeophyte $(\times$ Triticosecale $)$ to Italy are here recorded; among the 30 novelties and the 16 confirmed taxa to the flora of Lombardy, 6 are Italian endemics (Adenostyles australis, Brachypodium genuense, Centaurea aplolepa s.l., Hieracium grovesianum s.1., Stipa etrusca, Tephroseris italica).

Key words: Alien flora, Italian endemics, Northern Apennines, phytogeography, Po Plain.

\section{INTRODUZIONE}

Sino ad oggi la provincia di Pavia risultava una delle aree floristicamente meno conosciute dell'Italia nord-occidentale. Mentre quasi tutte le province limitrofe si sono dotate negli ultimi vent'anni di flore ed atlanti distributivi (ad es. Carrega \& Silla, 1995, 1996, 1999; Banfi \& Galasso, 1998; Bracchi \& Romani, 2010; Brusa \& Rovelli, 2010; Giordana, 2010b), lo stato delle conoscenze floristiche in questo territorio è rimasto fossilizzato alla prima metà dell'Ottocento, quando videro la luce Flora ticinensis (Nocca \& Balbis, 1816, 1821) e il Prospetto delle Piante fanerogame ritrovate nella Provincia Pavese (Rota, 1847), di fatto le uniche flore interamente dedicate alla provincia di Pavia. La storica lacuna conoscitiva trova un riscontro eloquente nella prima carta delle conoscenze floristiche in Italia (Filipello, 1978), in cui più del $78 \%$ del territorio provinciale, corrispondente alla Lomellina e all'Oltrepò Pavese, è classificato come "area con conoscenza generica appena informativa". La situazione rimane pressoché immutata 27 anni dopo, quando la nuova carta di Rossi et al. (2005) illustra un aumento di conoscenze solo per la parte nord del Parco del Ticino ("area ben conosciuta") e per il confine nordorientale con la provincia di Milano ("area abbastanza ben conosciuta"), mentre Lomellina e Oltrepò Pavese (con l'eccezione della zona del Monte d'Alpe) rimangono etichettati come "aree con conoscenza generica appena informativa".

In questa sede prende vita un progetto di aggiornamento e sintesi delle conoscenze floristiche in provincia di Pavia che mancava da oltre un secolo e mezzo. L'opera, come già annunciato da Ardenghi (2015a, 2015b), si articolerà in tre contributi distinti, ciascuno dedicato a uno 
dei tre macrosettori in cui è tradizionalmente suddiviso il territorio provinciale, ben definiti sotto il profilo storico, geografico e ambientale: Lomellina (porzione a nord del Po compresa tra la sponda occidentale del Ticino e il Sesia; include anche il Siccomario), Pavese (porzione a nord del Po compresa tra la sponda orientale del Ticino e il Lambro Meridionale) e Oltrepò Pavese (porzione a sud del Po) (Fig. 1). Quest'ultimo macrosettore è stato selezionato quale primo capitolo della serie ed è l'oggetto del presente articolo. Gli altri due contributi sono attualmente in fase di realizzazione da parte del primo autore.

\section{Geografia}

L'Oltrepò Pavese è la porzione più meridionale della provincia di Pavia e della Lombardia. Si estende a sud del fiume Po su una superficie di 1.099,66 km², delimitato a ovest e a sud dai crinali che dividono la Valle Staffora rispettivamente dalla Val Curone (Piemonte, provincia di Alessandria) e dalla Val Boreca (Emilia-Romagna, provincia di Piacenza). Il confine orientale corre lungo lo spartiacque tra la Val Tidone pavese e la Val Trebbia piacentina, e prosegue, a nord del Lago di Trebecco, lungo la dorsale che separa la Valle Versa e la Valle Bardonezza dalla Val Tidone piacentina (Medici, 1932; Massi, 1967) (Fig. 1).

Benché oggi appaiano ben definiti e in armonia con la morfologia del territorio, i limiti amministrativi oltrepa-

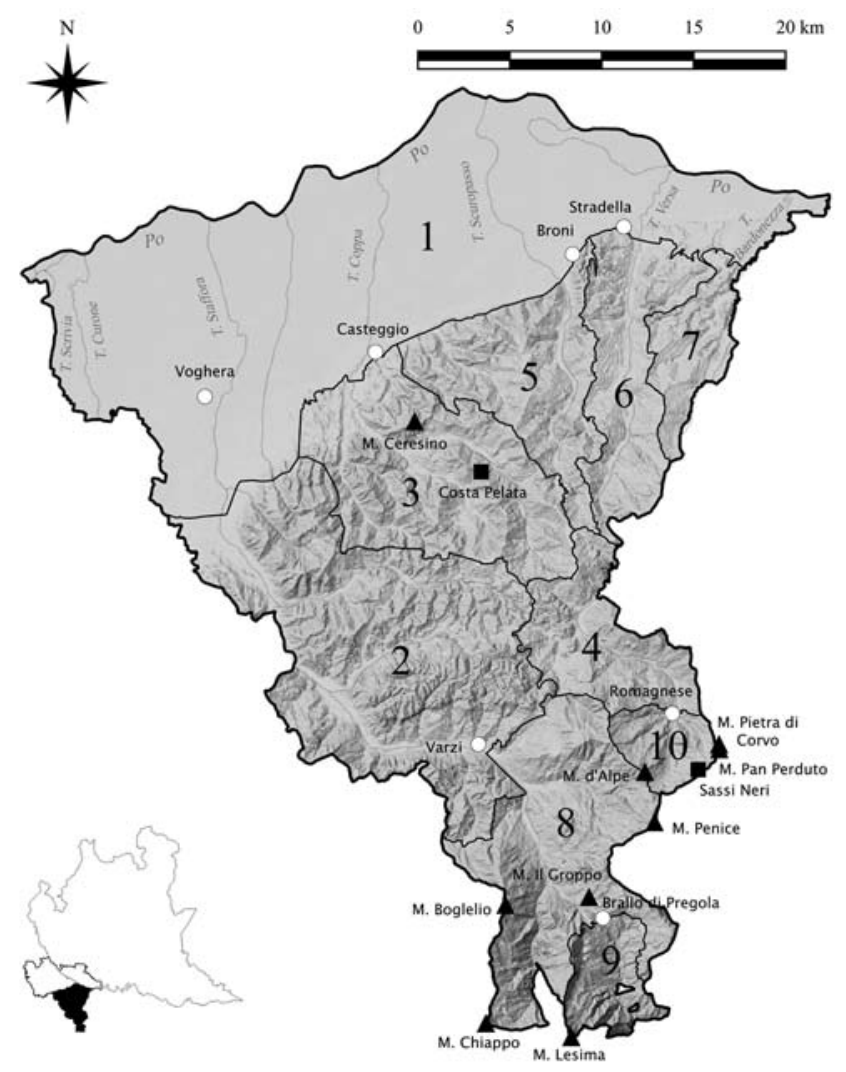

Fig. 1 - L'Oltrepò Pavese. Collocazione geografica e suddivisione in settori: 1) Pianura Padana; 2) bassa V. Staffora; 3) V. Coppa; 4) bassa V. Tidone; 5) V. Scuropasso; 6) V. Versa; 7) V. Bardonezza; 8) alta V. Staffora; 9) V. Avagnone; 10) alta V. Tidone (mappa realizzata in collaborazione con C. Ballerini). dani si sono assestati solamente alla fine degli anni '20 del secolo scorso, dopo numerose modifiche di natura politica e militare. Dal 1743 (trattato di Worms) al 1859 (decreto Rattazzi) il territorio dell'odierno Oltrepò Pavese era ripartito in due province distinte del Regno di Sardegna, la provincia di Voghera e la provincia di Bobbio (divenute, sotto la dominazione napoleonica, arondissement dei dipartimenti di Marengo, tra il 1799 e il 1805, e Genova, tra il 1805 e il 1814) (Regione Lombardia, 2002-2015). La sua estensione geografica era maggiore rispetto a quella dei giorni nostri in quanto comprensiva di località attualmente ricadenti nelle province di Piacenza (es. Bobbio, Caminata, Cerignale, Ottone, Zerba), Alessandria (es. Sale, Guazzora) e Genova (es. Fascia, Fontanigorda, Gorreto, Rovegno). Con il decreto Rattazzi del 1859, le due province vennero declassate a circondari della provincia di Pavia e nel 1923 gran parte del circondario di Bobbio fu annesso alla provincia di Piacenza (regio decreto 8 luglio 1923, n. 1726). A seguito della marcia su Bobbio (dicembre 1924), nel 1926 i comuni di Romagnese, Ruino e Zavattarello, passati nel 1923 a Piacenza in seno al circondario di Bobbio (regio decreto 8 luglio 1923, n. 1726), tornarono a Pavia (regio decreto 23 dicembre 1926, n. 2246). L'assetto definitivo dei confini amministrativi si raggiunse nel 1928, quando il comune di Groppo venne soppresso ed aggregato al comune alessandrino di Pozzol Groppo (regio decreto 10 agosto 1928, n. 1968).

\section{Geomorfologia e idrografia}

Circa un terzo dell'Oltrepò Pavese è occupato dalla bassa Pianura Padana, delimitata sul lato meridionale dai rilievi collinari e montani dell'Appennino Pavese, che costituiscono i restanti due terzi del territorio oltrepadano. L'Appennino Pavese rappresenta un prolungamento dell'Appennino Ligure, che raggiunge il suo estremo settentrionale in corrispondenza dello "Sperone di Stradella", situato a circa $5 \mathrm{~km}$ dalla sponda destra del Po.

L'altitudine varia da un minimo di $50 \mathrm{~m}$ lungo il corso del Po ad un massimo di $1.724 \mathrm{~m}$ sulla cima del Monte Lesima, il punto più elevato dell'Oltrepò Pavese. Tra le vette più alte, oltre al Monte Lesima, si ricordano i Monti Chiappo (1.699 m), Cima della Colletta (1.493 m), Boglelio (1.492 m), Terme (1.489 m), La Colla (1.438 m), Penice $(1.460 \mathrm{~m})$ e il Monte d'Alpe $(1.254 \mathrm{~m})$, alcuni dei quali condivisi con le province di Piacenza (Lesima, Penice), di Alessandria (Boglelio) o con entrambe (Chiappo).

L'Oltrepò Pavese è attraversato da un tratto del fiume Po, che per circa $50 \mathrm{~km}$ ne traccia il confine settentrionale. Lungo il suo percorso sfociano diversi torrenti appenninici, il più importante dei quali è lo Staffora, che per $58 \mathrm{~km}$ solca la valle principale dell'Oltrepò Pavese. Tra gli altri affluenti si ricordano, da ovest verso est, i torrenti Coppa, Scuropasso, Versa e Bardonezza, che definiscono una serie regolare di valli collinari ad andamento sud-nord. Sul versante settentrionale del Monte Penice nasce il torrente Tidone, che attraversa l'Oltrepò Pavese orientale fino alla sua immissione nel Lago di Trebecco (sul confine con Nibbiano, Piacenza), un bacino artificiale originatosi dopo la costruzione della diga del Molato nel 1928 (Comune di Nibbiano, 2015). All'estremità sud-orientale dell'Oltrepò Pavese il confine regionale è definito da un breve tratto del 
fiume Trebbia, nel quale si immette il torrente Avagnone; quest'ultimo incide una ripida valle ad andamento sud-est intersecante due enclavi interregionali (Lama e Valle Inferiore) del comune di Corte Brugnatella (Piacenza).

\section{Geologia}

Il variegato panorama geologico dell'Oltrepò Pavese rispecchia la complessa storia orogenetica dell'Appennino Settetrionale, durante la quale diverse unità tettoniche sono sovrascorse le une sulle altre.

Le rocce che costituiscono i rilievi dell'Appennino Pavese sono per la maggior parte di natura sedimentaria, differenti per genesi ed età. Lungo il margine sud della Pianura Padana, originata da alluvioni pleistoceniche ( $d i-$ luvium), un mosaico di formazioni mioceniche e plioceniche delinea i primi rilievi collinari, costituiti da conglomerati, arenarie (Arenarie di Monte Arzolo, Conglomerati di Cassano Spinola), argille (Argille di Lugagnano) e marne (Marne di Sant'Agata Fossili), sporadicamente compenetrati dalla Formazione Gessoso-Solfifera (marne, arenarie e conglomerati gessiferi), dalla quale hanno origine numerose sorgenti di acque sulfuree (cfr. Taramelli, 1916). Nella media-alta collina si osservano formazioni più antiche, come le Marne di Rigoroso (Oligocene-Miocene), le Arenarie di Monte Valassa e le Marne di Monte Lumello (Miocene) in bassa Valle Staffora, e le Arenarie di Renzano (Eocene-Oligocene) a cavallo tra la Valle Coppa e la Valle Versa. In quest'area sono particolarmente diffusi i litotipi eterogenei, come la Formazione di Val Luretta (nota anche come Calcare di Pietra dei Giorgi, risale al Paleocene-Eocene; si presenta come un'alternanza ritmica calcareo-marnoso-arenacea), componente principale dei colli tra l'alta Valle Versa e Montalto Pavese, e il Complesso Caotico (formazione a matrice argillosa inglobante frammenti eterogenei di rocce più rigide), osservabile specialmente su alcune dorsali della Valle Coppa (es. Costa Pelata). Le formazioni marnoso-argillose, decisamente instabili da un punto di vista idrogeologico, sono spesso interessate, soprattutto sui versanti più ripidi, da vistosi solchi di erosione prodotti dalle acque meteoriche, i calanchi. Tra i più imponenti si ricordano gli "orridi di Sant'Antonino" (Torrazza Coste), intagliati nelle Marne di Sant'Agata Fossili, e i calanchi di Valle di Nivione (Varzi), che solcano le Marne di Rigoroso.

Risalgono al Paleocene e al Cretacico superiore i flysch calcareo-marnosi, calcarenitici e arenacei che costituiscono la maggior parte dei rilievi montani, come il Monte Penice (Formazione di Monte Penice) e le dorsali dei monti Chiappo-Bogleglio, Lesima-Cima della Colletta (Calcari di Monte Antola) e Monte d'Alpe-Calenzone (Calcari di Monte Cassio). In questa zona si riscontra anche la presenza di altri litotipi, come le Arenarie di Scabiazza (stratificazioni di arenarie, marne e argille marnose risalenti al Cretacico superiore) e le Argille a palombini di Barberino (Cretacico inferiore). Da queste ultime emergono imponenti masse ofiolitiche, costituite in prevalenza da peridotiti serpentinizzate; localmente noti come "sassi neri" o "pietre nere", gli affioramenti di queste rocce sono circoscritti ad alcune aree dell'alta Valle Staffora (Monte Il Groppo, Santa Margherita di Staffora) e dell'alta Val Tidone (Monte Pietra di Corvo, Monte Pan Perduto, Sas- si Neri) (Bogliani et al., 2003; Carta Geologica d'Italia 1:100.000, fogli 59 e 71).

\section{Clima}

Secondo la classificazione di Köppen, il clima dell'Oltrepò Pavese rientra nella classe $\mathrm{C}$ dei climi temperati (Pinna, 1970). Il gradiente altitudinale, tuttavia, determina a livello locale una marcata diversificazione in termini di temperatura e precipitazioni, che consente di suddividere il territorio oltrepadano in due aree climatiche distinte: l'area planiziale-collinare caratterizzata da clima temperato subcontinentale e l'area montana con clima temperato fresco (Ottone \& Rossetti, 1980). Le temperature medie annue oscillano dai $12^{\circ} \mathrm{C}$ della Pianura Padana e della bassa Valle Staffora a valori di poco inferiori ai $5^{\circ} \mathrm{C}$ dei rilievi montani (Ottone \& Rossetti, 1980). Le precipitazioni medie annue presentano i valori più bassi, inferiori ai 750 $\mathrm{mm}$, in Pianura Padana, in bassa Valle Staffora e in Valle Coppa; raggiungono invece $i$ valori più alti, compresi tra i $1.400 \mathrm{~mm}$ e i $1.600 \mathrm{~mm}$, sui crinali della fascia montana (Rossetti \& Ottone, 1979), che subiscono l'influsso delle correnti umide provenienti dal vicino Mar Ligure (Bogliani et al., 2003). Il regime pluviometrico è di tipo sublitoraneo appenninico, caratterizzato da un massimo autunnale (che si verifica nel mese di ottobre in pianura e a novembre in collina e in montagna) prevalente rispetto a quello primaverile (maggio) e da un minimo estivo (luglio) superiore a quello invernale (gennaio) (Rossetti \& Ottone, 1979; Ottone \& Rossetti, 1980).

In base alla classificazione di Rivas-Martínez et al. (2015a), in Oltrepò Pavese si riconoscono due bioclimi: temperato continentale nella porzione planiziale e temperato oceanico sui rilievi collinari e montani. Il termotipo è mesotemperato in pianura e in collina; nella nella zona montana è invece di tipo supratemperato (Rivas-Martínez et al., 2015d). L'ombrotipo è umido in gran parte del territorio, ad eccezione della porzione orientale della Pianura Padana, dove si riscontra l'ombrotipo subumido (RivasMartínez et al., 2015c). È infine possibile riconoscere tre tipi di continentalità, che ricalcano fondamentalmente le tre fasce altitudinali: subcontinentale (pianura), semicontinentale (collina) ed euoceanico (montagna) (Rivas-Martínez et al., 2015b).

\section{Il paesaggio vegetale}

Secondo il recente schema biogeografico introdotto da Rivas-Martínez et al. (2004), l'Oltrepò Pavese ricade nella regione Eurosiberiana, subregione Alpino-Caucasica, provincia Appennino-Balcanica; è suddiviso in due settori, Padano e Appenninico, che coincidono rispettivamente con la porzione planiziale e i rilievi collinari-montani. Prendendo in considerazione sistemi di classificazione pregressi (Giacomini \& Fenaroli, 1958; Arrigoni, 1983) o alternativi (European Commission, 2006), appare costante il posizionamento del territorio oltrepadano a cavallo tra due regioni biogeografiche fortemente dissimili (quella Mediterranea e una "continentale" di cui l'Oltrepò Pavese fa parte, denominata, in base alla fonte considerata, Medio-Europea, Eurosibirica, Eurosiberiana o Continentale) e la sua ripartizione in due subunità distinte, corrispondenti essenzialmente alla Pianura Padana e all'Ap- 
pennino Pavese. La peculiare collocazione biogeografica dell'Oltrepò Pavese contribuisce in modo sostanziale alla diversità floristica e all'eterogeneità del paesaggio vegetale che lo contraddistinguono in ambito sia provinciale, sia regionale.

Da un punto di vista fitoclimatico, è possibile individuare tre piani altitudinali, ciascuno dei quali caratterizzato da parametri ambientali omogenei: planiziale o basale (50-300 m), collinare (301-900 m) e montano (901-1724 $\mathrm{m})$. Non è possibile individuare un piano subalpino, in quanto le comunità vegetali che dovrebbero differenziarlo sono estremamente frammentarie e localizzate in alcuni punti dei rilievi più elevati (Fig. 2).

Il piano planiziale è in larga parte occupato dalla bassa Pianura Padana, i cui terreni fertili hanno favorito nel corso dei secoli lo sviluppo di un variegato paesaggio agricolo, dove si alternano colture cerealicole (mais, frumento, orzo, grano duro, sorgo), foraggere (erba medica, festuca falascona, loiessa), oleaginose (soia, colza, girasole) e orticole (pomodoro, pisello, cipolla, zucca), tra le quali spiccano alcune antiche cultivar locali (o landrace; cfr. Camacho Villa, 2005) riscoperte negli ultimi anni, come la 'Zucca berrettina di Lungavilla', il 'Peperone di Voghera', la 'Cipolla dorata di Voghera' (Camera di Commercio Pavia, 2009; ERSAF, 2010; Cavagna et al., 2012; Associazione Zucca Berrettina, 2014; Contardi, 2015) e la 'Zucca da mostarda', una cultivar di Lagenaria siceraria (Molina) Standl., diffusa anche in alcune zone della bassa pianura emiliana (cfr. Piazza, 2014), impiegata in passato per la produzione della mostarda di Voghera (M. Orlandini, com. pers. a G. Rossi, 2016) (Fig. 3). Benché

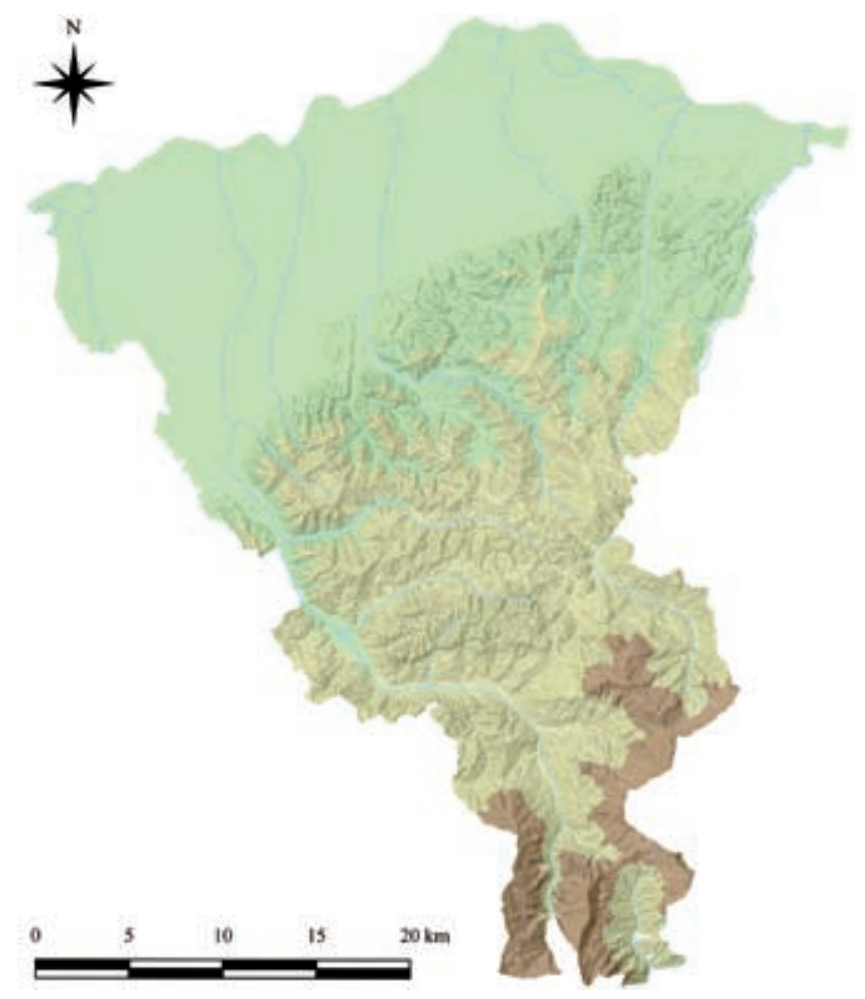

Fig. 2 - I piani altitudinali dell'Oltrepò Pavese: planiziale, 50-300 m (verde); collinare, 301-900 m (beige); montano, 901-1724 m (marrone) (mappa realizzata in collaborazione con C. Ballerini). gli aspetti monocolturali siano più attenuati rispetto alla limitrofa pianura lomellina e pavese (dove risicoltura e maiscoltura intensive sono dominanti), l'espansione agricola ha comunque compromesso in modo permanente la vegetazione naturale, con gravi ripercussioni soprattutto sulle cenosi forestali.

Degli antichi boschi alluvionali permangono solo alcuni frammenti residuali, costituiti in prevalenza da Quercus robur L. subsp. robur e Ulmus minor Mill. subsp. minor (Ulmenion minoris Oberd. 1953), mentre un unico esempio di ontaneta ad Alnus glutinosa (L.) Gaertn. (Alnion glutinosae Malcuit 1929) sopravvive all'interno del SIC IT2080020 "Garzaia della Roggia Torbida" di Bressana Bottarone, uno dei due siti di importanza comunitaria presenti in Oltrepò Pavese (Perracino, 2010). Il denudamento dei suoli ha invece favorito l'insediamento e l'espansione di formazioni boschive secondarie dominate da Robinia pseudoacacia L., alla quale si associa, in condizioni più xeriche, Ailanthus altissima (Mill.) Swingle (ChelidonioRobinietalia Jurko ex Hadac \& Sofron 1980). I robinieti si estendono fino alla sommità del piano collinare, colonizzando i margini e le chiarie dei boschi, le scarpate delle vie di comunicazione e i terreni abbandonati. La situazione di degrado è analoga anche nelle golene del Po, dove la vegetazione riparia è rappresentata da lembi di saliceto a Salix alba L. (Salicion albae Soó 1930) scampati alla pioppicoltura industriale, spesso frammisti a neofite arboree, come Populus $\times$ canadensis Moench e Acer negundo L. I saliceti arbustivi a Salix cinerea L. e Viburnum opulus L. [Salici cinereae-Viburnion opuli (Passarge 1985) de Foucault 1991], divenuti ormai una rarità, sono in larga parte rimpiazzati da formazioni monofitiche ad Amorpha fruticosa L., a cui si intercalano megaforbieti nitro-igrofili (Convolvuletalia sepium Tüxen ex Mucina 1993), veri e propri grovigli di neofite invasive, soprattutto lianose [Humulus japonicus Siebold \& Zucc., Parthenocissus inserta (A.Kern.) Fritsch, Sicyos angulatus L., Solidago gigantea Aiton, Symphyotrichum lanceolatum (Willd.) G.L.Nesom, Vitis riparia Michx., V. $\times$ koberi Ardenghi, Galasso, Banfi \& Lastrucci], in fase espansiva anche lungo i tratti pedecollinari dei torrenti appenninici. La aliene invasive costituiscono la componente principale delle comunità pioniere dei greti sabbiosi (localmente noti come "sabbioni" o "spiaggioni") e delle sponde fangose (Bidentetalia tripartitae Br.-Bl. \& Tüxen ex Klika in Klika \& Hadac 1944) del Po, dove abbondano, accanto alle autoctone Echinochloa crus-galli (L.) P.Beauv. e Persicaria lapathifolia (L.) Delarbre subsp. lapathifolia, Amaranthus tuberculatus (Moq.) J.D.Sauer, Bidens frondosa L., Cyperus sp. pl., Oenothera sp. pl., Panicum sp. pl. e Xanthium italicum Moretti.

Gli effetti più incisivi dell'antropizzazione si registrano nella fascia pedecollinare, l'area dell'Oltrepò Pavese più densamente popolata e maggiormente interessata dall'espansione edilizia, che prosegue incessante con la costruzione di nuovi centri commerciali e logistici; qui si concentrano i più grandi abitati (Voghera, Stradella, Broni, Casteggio) e le principali vie di comunicazione (l'Autostrada A21 Torino-Brescia, la ex Strada Statale SS10 Padana Inferiore e la linea ferroviaria AlessandriaPiacenza), che favoriscono un continuo ingresso di nuo- 


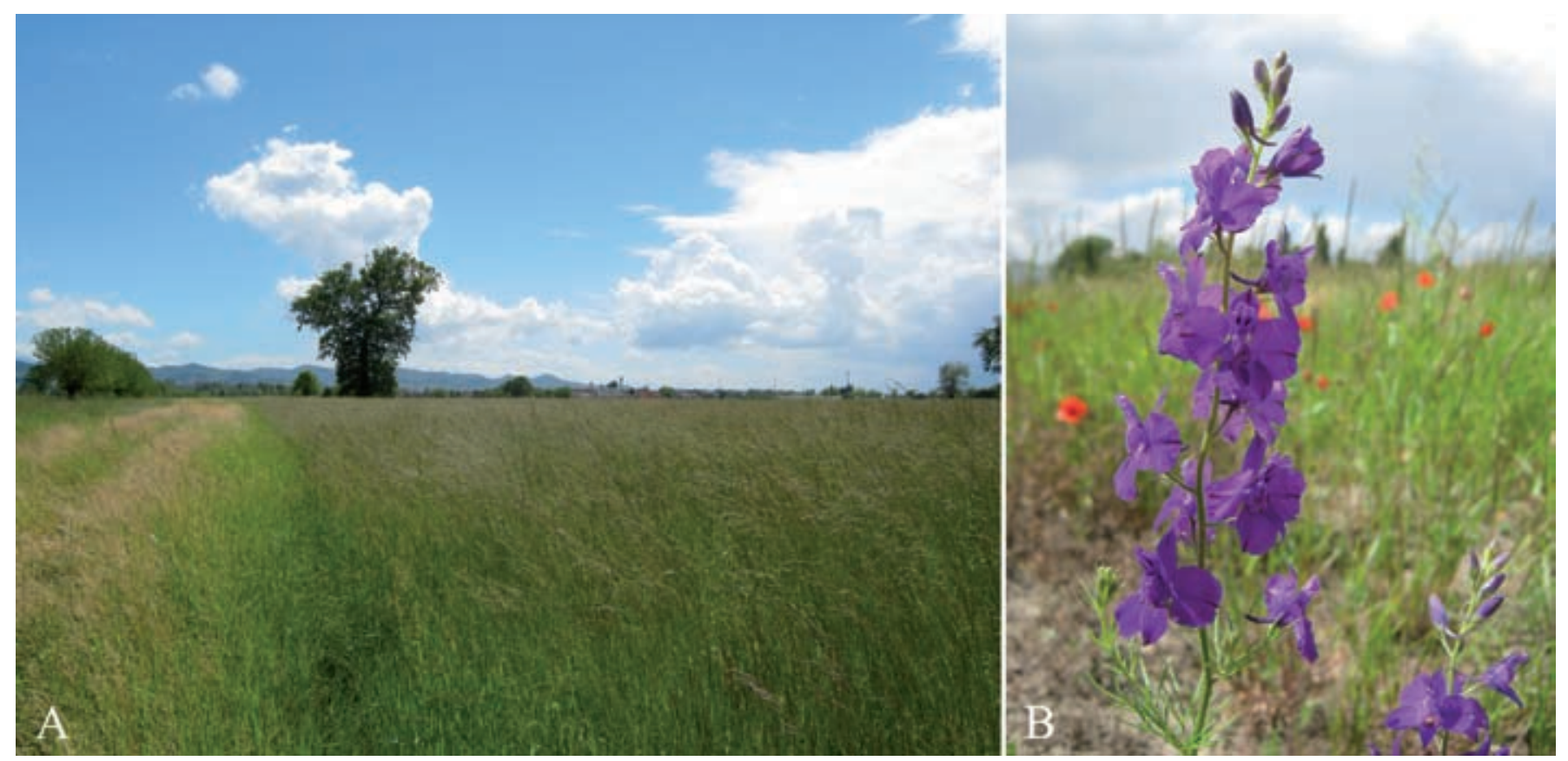

Fig. 3 - Aspetti agricoli in Pianura Padana (Bressana Bottarone): A) campo di festuca falascona [Lolium arundinaceum (Schreb.) Darbysh. subsp. arundinaceum]; B) Consolida orientalis (J.Gay) Schrödinger presso un campo di frumento (Foto: Nicola M.G. Ardenghi).

ve specie esotiche. Nelle aree ferroviarie, in particolare, a partire dal secondo dopoguerra si è progressivamente instaurata una flora del tutto esclusiva, caratterizzata da entità di origine mediterranea (es. Geranium purpureum Vill., Verbascum sinuatum L., Veronica cymbalaria Bodard subsp. cymbalaria) e alloctona [Bidens bipinnata L., Cyperus schweinitzii Torr., Euphorbia davidii Subils, Mirabilis nyctaginea (Michx.) MacMill., Setaria faberi R.A.W.Herrm.] (Fig. 4) introdotte dai convogli di passaggio (cfr. Viola, 1952; Ardenghi, 2013a).

Per la natura dei substrati, le opere di bonifica e la regimazione dei corsi d'acqua, in tutto l'Oltrepò Pavese le zone umide sono estremamente localizzate e di dimensioni esigue. In pianura, la Lanca d'Enzo (Fig. 5A) rappresenta sicuramente uno degli ambienti più interessanti dal punto di vista floristico: si tratta di una morta situata a nord dell'abitato di Portalbera, facente parte della ZPS IT2080701 "Po da Albaredo Arnaboldi ad Arena Po", l'unica zona di protezione speciale dell'Oltrepò Pavese (Casale et al., 2011). Sebbene l'abbandono di rifiuti al suo interno abbia determinato la scomparsa dell'unica stazione oltrepadana di Nymphoides peltata (S.G.Gmel.) Kuntze, questa morta continua ad ospitare pregevoli specie anfibie [come Butomus umbellatus L., Oenanthe aquatica (L.) Poir., Persicaria amphibia (L.) Delarbre], assenti o molto rare altrove; nelle immediate vicinanze sopravvive l'unica popolazione di Leucojum aestivum L. subsp. aestivum accertata in Oltrepò Pavese, la cui presenza è stata confermata solo di recente (Fig. 5C). Di analoga rilevanza è il Parco locale di interesse sovracomunale "Le Folaghe" di Casei Gerola, comprendente una serie di ex cave a fossa (frutto dell'attività estrattiva dell'argilla, molto diffusa fino alla metà del secolo scorso nella pianura centro-occidentale) alimentate da acqua di falda, che hanno acquisito nel tempo caratteristiche di naturalità (Amici del Parco Le Folaghe, 2009). In alcuni di questi bacini, di considerevole interesse ornitologico, si trovano diverse macrofite, alcune delle quali esclusive di questo sito, come Utricularia australis R.Br., Ranunculus trichophyllus Chaix subsp. trichophyllus e Potamogeton nodosus L.

Non è rimasta alcuna traccia della comunità alofila a Salicornia perennans Willd. subsp. perennans e Sporobolus aculeatus (L.) P.M.Peterson (Crypsidion aculeatae Pignatti 1954) che popolava la sorgente di acqua salsa (o cloruro-sodica) nei dintorni della Cascina Borrone di Mezzanino (e non Campospinoso, come tradizionalmente riportato da tutte le fonti floristiche), presumibilmente annientata da bonifiche avvenute nei primi decenni del Novecento. La stazione, con caratteristiche molto simili a quelle di Miradolo Terme (fagocitata dagli stabilimenti termali) e di Sermide nel Mantovano (cfr. Cesati, 1844; Bertolani-Marchetti, 1954; Galasso, 2015a; Truzzi, 2015), venne scoperta attorno al 1785 dallo speziale bronese Francesco Fumagalli (Moretti, 1822) e visitata in tempi successivi da svariati botanici che assistettero ad una graduale riduzione del contingente alofilo, già completamente estinto all'epoca dei tre sopralluoghi effettuati da Bertolani-Marchetti nel 1951 (Bertolani-Marchetti, 1954). Giulietti (1900) e Taramelli (1916) testimoniano la presenza di acque salse anche a Casteggio (nella cantina dell'Albergo Vittoria), Santa Giuletta ("Cascina Saline", ovvero Ca' Salini), Barbianello e Rivanazzano Terme, lasciando supporre una maggior diffusione di questa fitocenosi nel passato.

A differenza della pianura e dei primi rilievi a ridosso di quest'ultima, il piano collinare è caratterizzato da una buona copertura boschiva. Sui substrati calcarei, marnosi e argillosi prevalgono i boschi termofili a Quercus pube- 


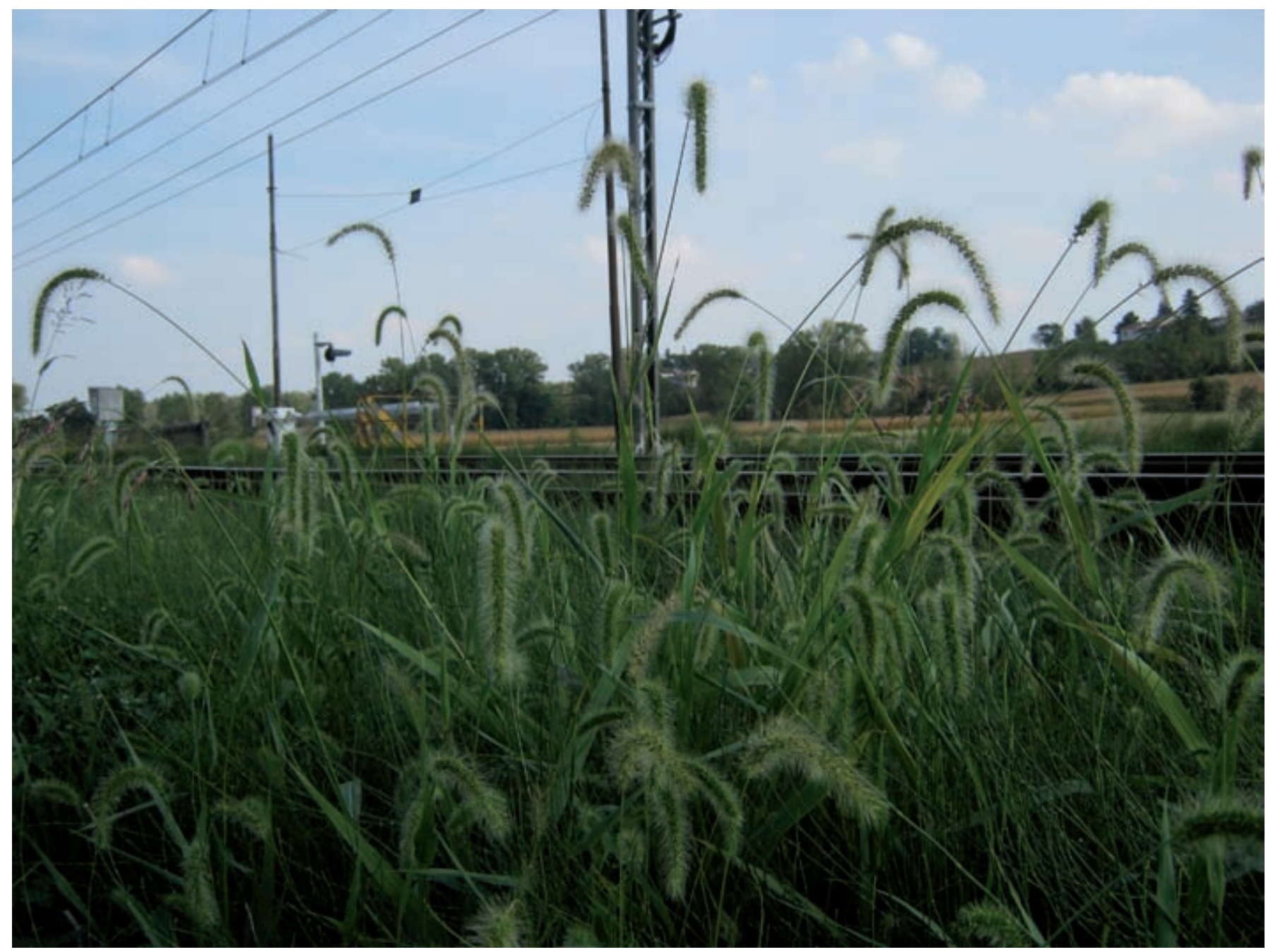

Fig. 4 - Setaria faberi R.A.W.Herrm. sulla massicciata ferroviaria ad Arena Po; negli ultimi anni questa neofita è in espansione lungo la linea Alessandria-Piacenza (Foto: Nicola M.G. Ardenghi).

scens Willd. subsp. pubescens, spesso in associazione con Ostrya carpinifolia Scop. e Fraxinus ornus L. subsp. ornus (Carpinion orientalis Horvat. 1958), che diventano a loro volta dominanti nelle forme destrutturate del bosco. Uno degli esempi più rappresentativi di questa fitocenosi si trova appena sopra l'abitato di Casteggio, sul Monte Ceresino (Calvignano), la cui flora, ricca in nemorali quasi del tutto scomparse nel resto della prima collina [es. Anemonoides trifolia (L.) Holub subsp. brevidentata (Ubaldi \& Puppi) Galasso, Banfi \& Soldano, A. ranunculoides (L.) Holub, Doronicum pardalianches L., Physospermum cornubiense (L.) DC., Erythronium dens-canis L., Chamaeiris graminea (L.) Medik., Sanicula europaea L., Vinca minor L., Polygonatum odoratum (Mill.) Druce, Cardamine bulbifera (L.) Crantz, Euphorbia dulcis L., Mercurialis perennis L., Melittis melissophyllum L. subsp. melissophyllum, Viola alba Besser subsp. dehnhardtii (Ten.) W.Becker], è rimasta pressoché intatta dai primi anni dell'Ottocento. Sui suoli più acidi i querco-ostrieti sono localmente sostituiti dai castagneti, che possono ospitare una limitata presenza di Quercus petraea (Matt.) Liebl. subsp. petraea, $Q$. pubescens subsp. pubescens e Torminalis clusii (L.) K.R.Robertson \& J.B.Phipps nello strato arboreo, e Corylus avellana L. in quello arbustivo [Erythronio dens-canis-Quercion petraeae Ubaldi (1988) 1990]. Con ogni probabilità i castagneti non rappresentano una formazione indigena dell'Appennino Settentrionale, ma sono il frutto di un'introduzione secondaria avvenuta in epoca romana per lo sfruttamento, oggi largamente abbandonato, del legname e delle castagne. Sui substrati arenacei, in condizioni più mesofile, si rinvengono cerrete acidofile, caratterizzate da strato arboreo dominato da Quercus cerris L., a cui si affiancano $Q$. pubescens subsp. pubescens, Fraxinus ornus subsp. ornus e Torminalis clusii (Erythronio dens-canis-Quercion petraeae) (Pignatti, 1982; Andreis \& Sartori, 2011; Biondi \& Blasi, 2015).

In assenza di copertura arborea, sui substrati marnosi e argilloso-arenacei si sviluppano praterie xerofile, generalmente di origine secondaria, costituite in prevalenza da emicriptofite [es. Bromopsis erecta (Huds.) Fourr. subsp. erecta, Brachypodium rupestre (Huds.) Roem. \& Schult., Festuca trichophylla (Ducros ex Gaudin) K.Richt. subsp. trichophylla, Koeleria macrantha (Ledeb.) Schult. subsp. macrantha, Hippocrepis comosa L. subsp. comosa, Anthyllis vulneraria L. subsp. polyphylla (DC.) Nyman, Centaurea jacea L. subsp. gau- 

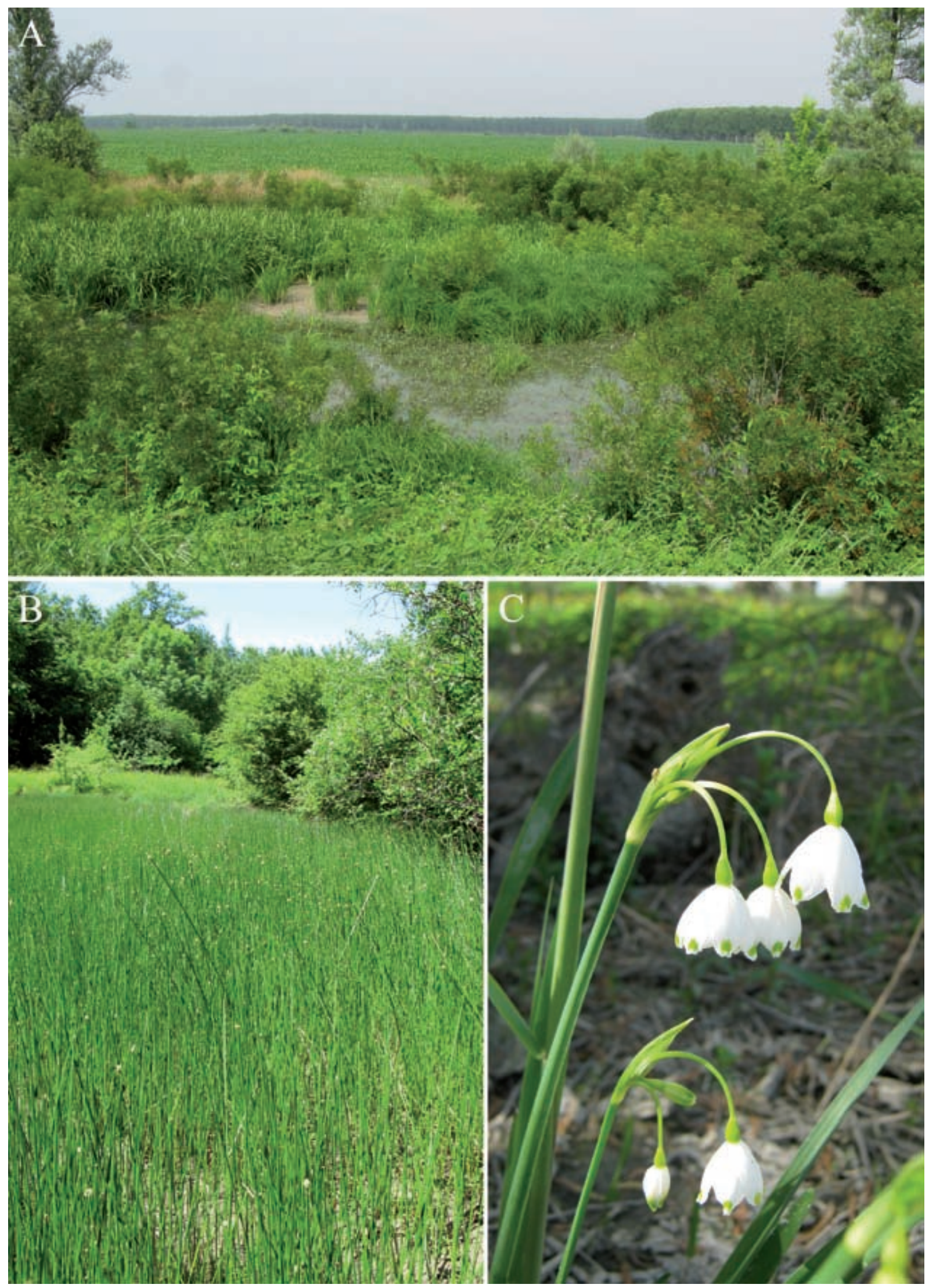

Fig. 5 - Zone umide: A) Lanca d'Enzo (Spessa, sponda destra del Po), con Persicaria amphibia (L.) Delarbre (al centro della morta) e Acorus calamus L. frammisto a Carex acuta L. (sponda, a sinistra) (Foto: Francesco Polani); B) Gabiàs (Romagnese), stagno con Eleocharis palustris (L.) Roem. \& Schult. subsp. palustris (Foto: Francesco Polani); C) Leucojum aestivum L. subsp. aestivum sulle sponde del Lancone (Portalbera) (Foto: Nicola M.G. Ardenghi). 
dinii (Boiss. \& Reut.) Gremli], a cui può associarsi un numero limitato di camefite [es. Globularia bisnagarica L., Thymus oenipontanus Borbás, Coronilla minima L. subsp. minima, Lotus herbaceus (Vill.) Jauzein] (Bromion erecti W.Koch 1926). Molte di queste formazioni, per il ricco contingente di orchidee che accolgono [es. Ophrys holosericea (Burm.f.) Greuter subsp. holosericea, O. benacensis (Reisigl) O.Danesch, E.Danesch \&
Ehrend., Anacamptis pyramidalis (L.) Rich., Gymnadenia conopsea (L.) R.Br., Himantoglossum adriaticum H.Baumann], sono attribuibili all'habitat prioritario 6210(*) "Formazioni erbose secche seminaturali e facies coperte da cespugli su substrato calcareo (FestucoBrometalia) (*stupenda fioritura di orchidee)" della dir. n. 92/43/CEE (Direttiva "Habitat") (Abeli et al., 2012; Biondi \& Blasi, 2015) (Fig. 6). Praterie mesofile, regolar-
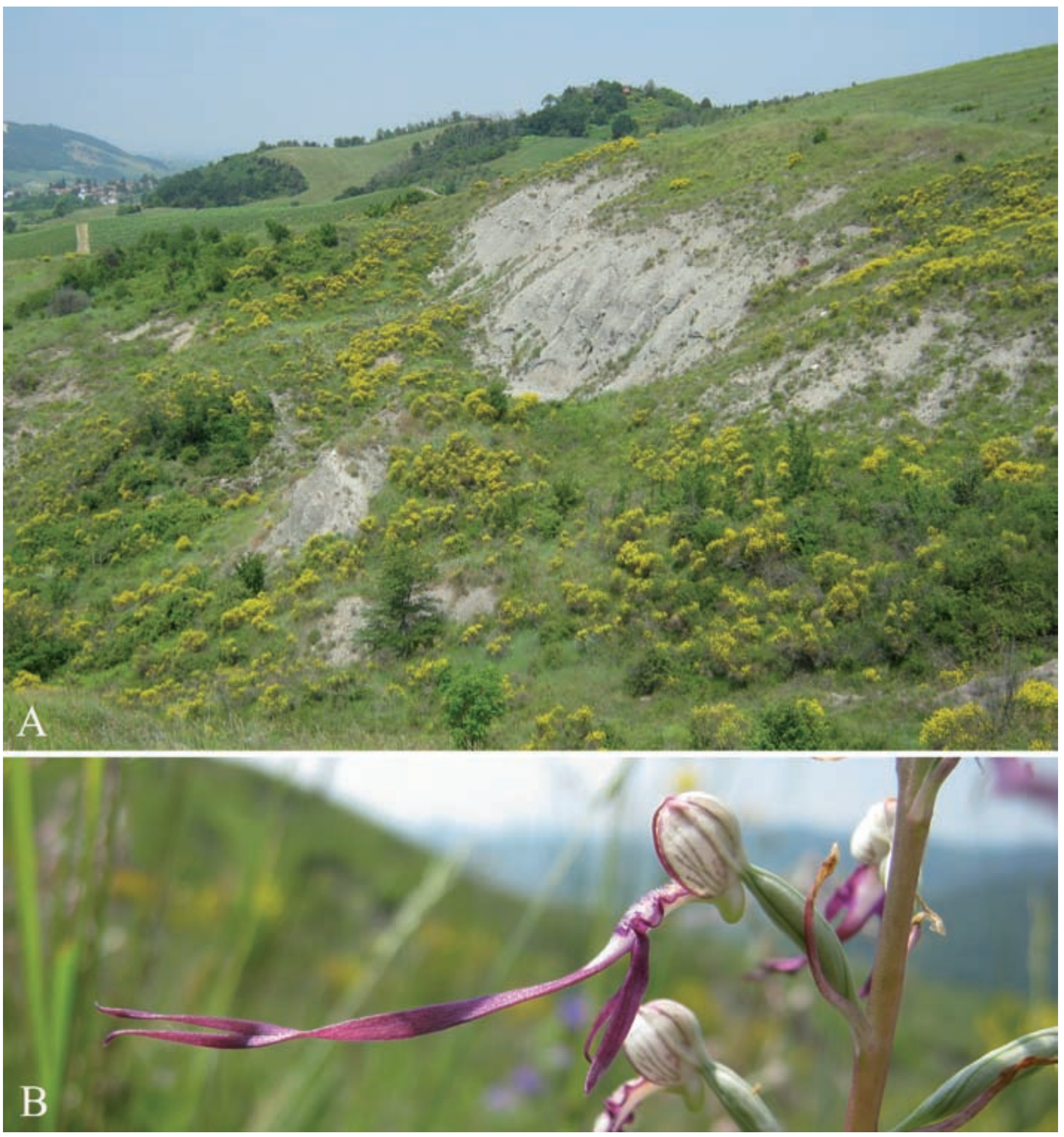

Fig. 6 - Aspetti naturali in Valle Coppa (Costa Pelata, Borgoratto Mormorolo): A) praterie xerofile, ginestreti a Spartium junceum L. e scarpate calanchive; B) Himantoglossum adriaticum H.Baumann, specie dell'Allegato II della dir. n. 92/43/CEE (Direttiva "Habitat") (Foto: Nicola M.G. Ardenghi). 
mente sfalciate e saltuariamente concimate, sono invece diffuse su suoli più profondi e con maggior disponibilità di acqua, dalla fascia collinare medio-alta a quella montana; qui la presenza di Bromopsis erecta subsp. erecta si riduce sensibilmente fino ad annullarsi, lasciando spazio ad emicriptofite più mesofile, come Arrhenatherum elatius (L.) P.Beauv. ex J.Pres1 \& C.Presl subsp. elatius, Dactylis glomerata L. subsp. glomerata, Trisetaria flavescens (L.) Baumg. subsp. flavescens, Lolium pratense (Huds.) Darbysh., Phleum pratense L., Onobrychis viciifolia Scop., Trifolium pratense L. subsp. pratense e Crepis vesicaria L. subsp. taraxacifolia (Thuill.) Thell. (Arrhenatheretalia elatioris Tüxen 1937).

Alcuni aspetti degli xerobrometi si ripresentano nelle formazioni calanchive e sui substrati conglomeratici, calcareo-marnosi e arenacei affioranti, dove le camefite [es. Artemisia alba Turra, Astragalus monspessulanus L. subsp. monspessulanus, Teucrium montanum L., Fumana procumbens (Dunal) Gren. \& Godr., Globularia bisnagarica, Anthericum liliago L., Dianthus longicaulis Ten., Argyrolobium zanonii (Turra) P.W.Ball. subsp. zanonii, Helichrysum italicum (Roth) G.Don subsp. italicum, Staehelina dubia L., Helianthemum nummularium (L.) Mill., H. apenninum (L.) Mill. subsp. apenninum, Cleistogenes serotina (L.) Keng subsp. serotina, Sedum thartii L.P.Hébert, Convolvulus cantabrica L.] diventano prevalenti sulla componente emicriptofitica [rappresentata principalmente da Bromopsis erecta subsp. erecta, Festuca inops De Not., Brachypodium rupestre, Saponaria ocymoides L. subsp. ocymoides e Asperula purpurea (L.) Ehrend. subsp. purpurea], dando origine a vere e proprie garighe camefitiche (Astragalenion monspessulani Biondi, Allegrezza \& Zuccarello 2005); negli stadi pionieri, specialmente sui substrati argillosi, possono insediarsi comunità di emicriptofite dell'alleanza Agropyrion pungentis Géhu 1968 em. 1973, in cui domina Elymus acutus (DC.) M.-A.Thiébaud, a cui sovente si associano Dittrichia viscosa (L.) Greuter subsp. viscosa e Galatella linosyris (L.) Rchb.f. subsp. linosyris (Volk, 1958; Gentile \& Sartori, 1975; Biondi \& Blasi, 2015). Formazioni terofitiche a carattere distintamente termofilo e subnitrofilo, riconducibili alla classe Thero-Brometalia (Rivas Goday \& Rivas-Martínez ex Esteve 1973) O.Bolòs 1975, si possono osservare in diversi contesti ambientali, a partire dai cigli stradali dei piani collinare e planiziale, costeggiati in primavera da abbondanti graminacee ruderali, tra cui Anisantha madritensis (L.) Nevski, $A$. diandra (Roth) Tutin ex Tzvelev, Avena barbata Pott ex Link subsp. barbata, A. sterilis L. subsp. ludoviciana (Durieu) Gillet \& Magne, Festuca danthonii Asch. \& Graebn. e Dasypyrum villosum (L.) P.Candargy, quest'ultima in evidente fase espansiva. In alcune località della media collina (ad es. Costa Pelata), pratelli mediterranei a Triticum vagans (Jord. \& Fourr.) Greuter, T. neglectum (Req. ex Bertol.) Greuter, Scorpiurus subvillosus L., Podospermum laciniatum (L.) DC. subsp. laciniatum, Coronilla scorpioides (L.) W.D.J.Koch e Trifolium angustifolium L. subsp. angustifolium (Fig. 7), spesso frammisti a Brachypodium distachyon (L.) P.Beauv., colonizzano le scarpate calanchive più esposte e si insinuano lungo i margini xerici dei campi, contribuendo a delineare i tipici mosaici agroambientali dell'Oltrepò Pavese collinare, dove campi di cereali, medicai e vigneti si alternano a prati aridi, arbusteti e lembi di querco-ostrieto (Fig. 8).

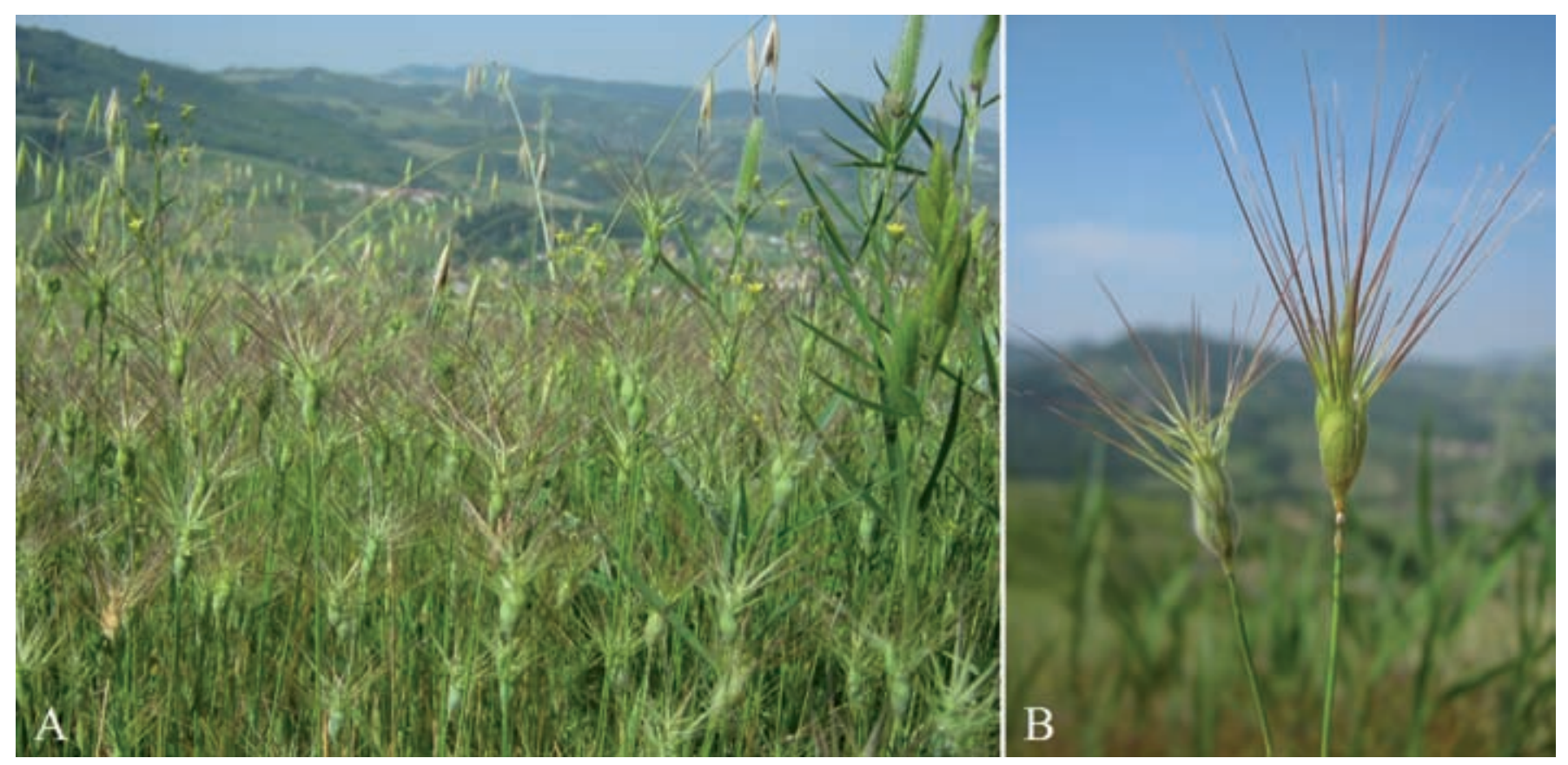

Fig. 7 - Comunità terofitiche mediterranee in Valle Coppa (Costa Pelata, Borgoratto Mormorolo): A) pratello con Triticum vagans (Jord. \& Fourr.) Greuter, Trifolium angustifolium L. subsp. angustifolium, Linum corymbulosum Rchb. e Avena sterilis L. subsp. ludoviciana (Durieu) Gillet \& Magne; B) Triticum vagans, a sinistra, e T. neglectum (Req. ex Bertol.) Greuter, a destra (Foto: Nicola M.G. Ardenghi). 


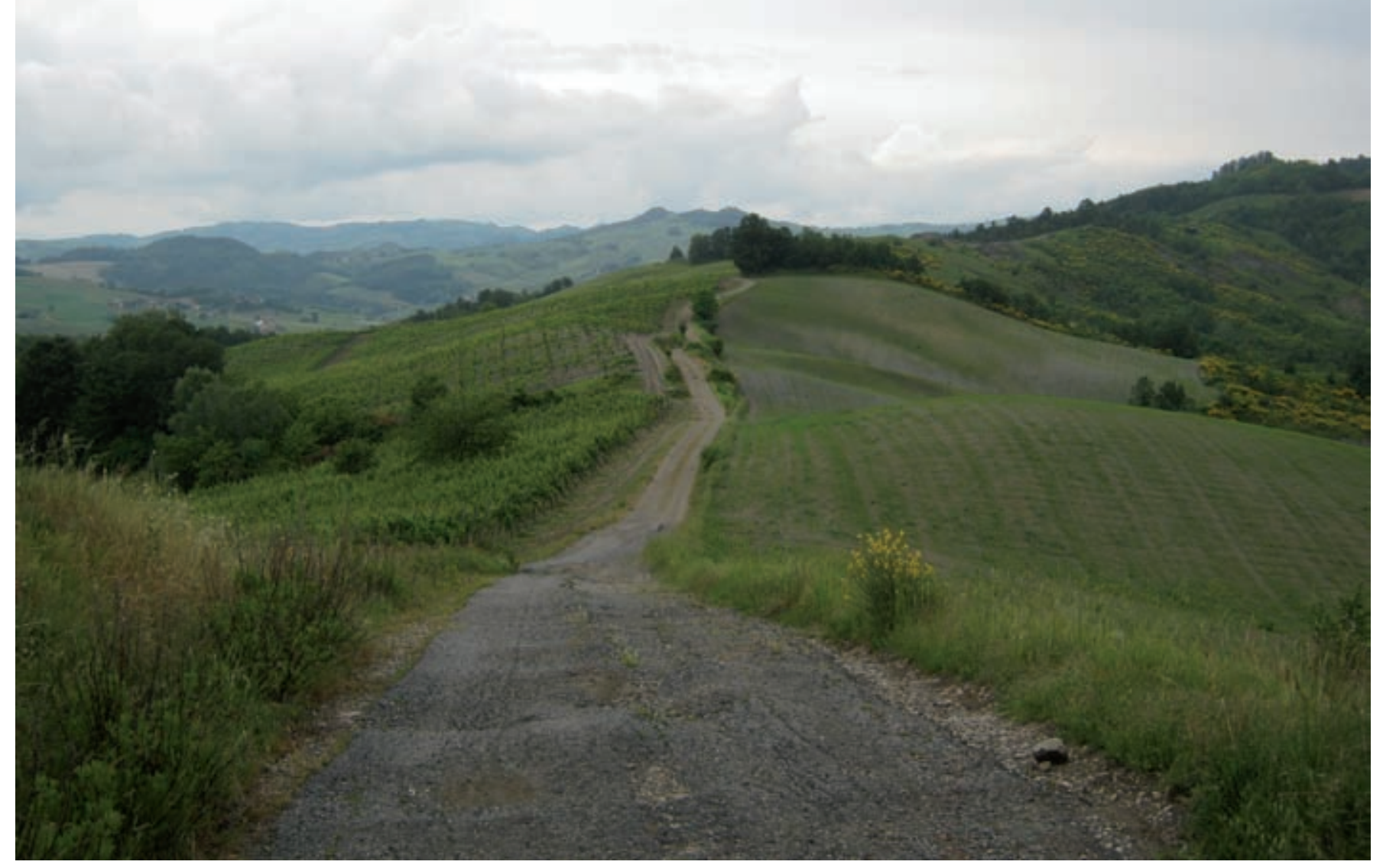

Fig. 8 - Tipico mosaico agroambientale oltrepadano a Costa Pelata (Borgoratto Mormorolo) (Foto: Nicola M.G. Ardenghi).

Così come in pianura, anche in collina sono diffuse le colture cerealicole, in particolare frumento e orzo, a cui nel passato si affiancavano avena, segale e alcune cultivar locali di mais da polenta ('Malga rüsa' e 'Vot ter', in italiano 'Marano' e 'Otto file': A. Albanesi, in verbis 15.12.2015), la componente base, assieme ad altre orticole (come i ' $\mathrm{Fa}$ sò dal diaul', un'antica cultivar di Phaseolus coccineus L.: A. Albanesi, in verbis 15.08.2015), di un'agricoltura di sussistenza oggi scomparsa. I campi di cereali del piano collinare si impostano su suoli più basici rispetto a quelli planiziali e annoverano tuttora una vegetazione segetale di grande pregio (Caucalidion platycarpi Tüxen ex von Rochow 1951), frutto di tecniche colturali meno intensive e del variegato contesto ambientale entro cui sono inseriti. Tra le infestanti più caratteristiche si ricordano Euphorbia falcata L. subsp. falcata, Bifora radians M.Bieb., Bunium bulbocastanum L., Ranunculus arvensis L., Melampyrum arvense L. subsp. arvense e Consolida regalis Gray subsp. regalis, alle quali si aggiungono specie più rare, ma non del tutto sparite, come Cyanus segetum Hill, Agrostemma githago L., Allium rotundum L., Adonis sp. pl., Bupleurum rotundifolium L. e Thymelaea passerina (L.) Coss. \& Germ. (Pignatti, 1957).
Nel panorama agricolo dell'Oltrepò Pavese primeggia la viticoltura, che vanta in quest'area geografica una tradizione millenaria. La coltivazione della vite è diffusa in tutta la media-bassa collina e si estende su una superficie di 13.269 ettari, pari a circa il 55\% della superficie vitata regionale (Fig. 9); in Valle Staffora, nella fascia collinare medio-alta, il vigneto è sostituito dai frutteti, con produzione di mele, pesche e ciliegie. Dai primi del Novecento la viticoltura ha gradualmente assunto caratteri intensivi, soprattutto nelle valli orientali (Bardonezza e Versa), zone di eccellenza per la produzione del vitigno 'Croatina', che costituisce la componente base del vino oltrepadano più celebre, il Bonarda. 'Croatina', 'Barbera', 'Pinot Nero', 'Riesling' e 'Moscato Bianco' sono diventati i vitigni principali dell'Oltrepò Pavese solo nel Ventesimo secolo, dopo l'avvento di oidio, peronospora e fillossera; furono infatti selezionati per la maggiore resistenza alle malattie crittogame e la migliore affinità d'innesto, a scapito di altri vitigni un tempo più comuni e oggi pressoché estinti (ad es. la 'Moradella') (Camera di Commercio Pavia, 2009; Maffi, 2010). In concomitanza, i portainnesti, rappresentati da specie americane "pure" o ibridate (con altre viti americane oppure con la stessa Vitis vinifera L.), si sono 


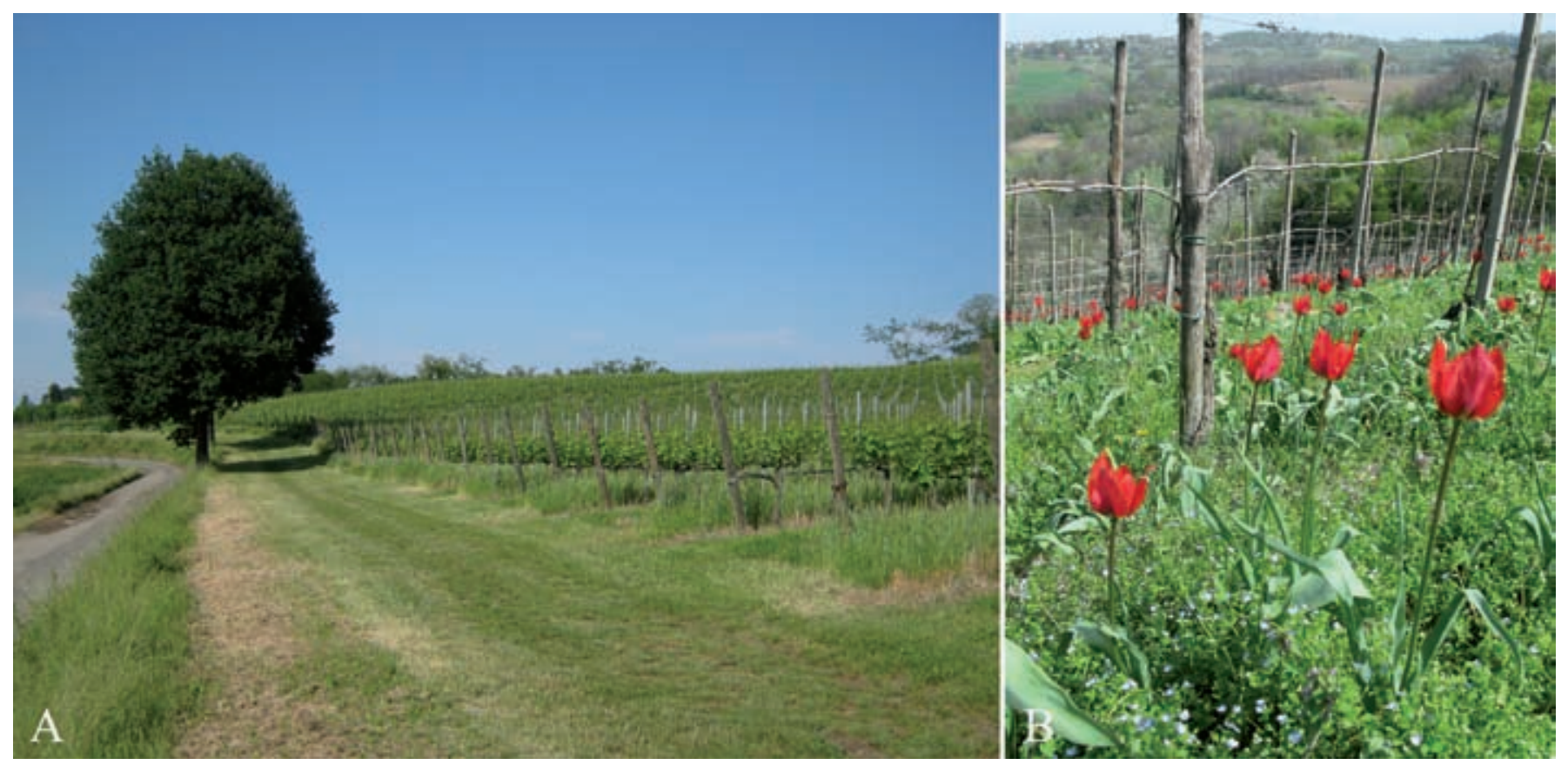

Fig. 9 - I vigneti oltrepadani: A) filari di 'Pinot Nero' in Valle Versa (San Zeno, Stradella) (Foto: Nicola M.G. Ardenghi); B) Tulipa raddii Reboul a Golferenzo (Foto: Francesco Polani).

autonomamente svincolati dal vigneto, insediandosi negli incolti, negli arbusteti e nelle formazioni boschive ripariali. Per la sua radicata tradizione vitivinicola, l'Oltrepò Pavese rappresenta quindi anche una delle aree italiane ed europee con la maggior concentrazione di viti alloctone naturalizzate e invasive, due delle quali con locus classicus situato proprio in terra oltrepadana: $V$. $\times$ ruggerii Ardenghi, Galasso, Banfi \& Lastrucci (Montù Beccaria) e $V$. × goliath Ardenghi, Galasso \& Banfi (Stradella) (Ardenghi et al., 2014, 2015). Così come altre colture, anche i vigneti ospitano una flora segetale piuttosto diversificata, la cui composizione varia in base al tipo di gestione: mentre il numero di infestanti tende ad annullarsi quando il suolo viene inerbito con graminacee [l'entità più utilizzata è Festuca rubra L. subsp. juncea (Hack.) K.Richt.], la sarchiatura sembra invece promuovere la presenza di geofite [Tulipa sylvestris L., T. raddii Reboul (Fig. 9B), Eranthis hyemalis (L.) Salisb.], alcune delle quali in forte rarefazione. Non mancano anche qui entità alloctone, come Brassica rapa L., Crepis sancta (L.) Bornm. subsp. nemausensis (P.Fourn.) Babc. e Diplotaxis erucoides (L.) DC. subsp. erucoides, quest'ultima introdotta sessant'anni fa dall'Italia mediterranea (Ardenghi \& Parolo, 2012; Ardenghi \& Mossini, 2013).

Il piano montano è occupato da faggete mesofile neutrobasifile (Trochiscantho-Fagetum sylvaticae Gentile 1975), localizzate su substrati arenacei, marnosi o calcarei. Lo strato arboreo è dominato da Fagus sylvatica L. subsp. sylvatica, mentre quello erbaceo è costituito da Trochiscanthes nodiflora (All.) W.D.J.Koch, Geranium nodosum L., Valeriana tripteris L. subsp. tripteris e da due endemiti italiani, Adenostyles australis (Ten.) Iamonico \& Pignatti e Phyteuma ovatum Honck. subsp. pseudospicatum Pignatti; a quote inferiori (800-1300 m), su suoli più sottili e xerici, nel sottobosco prevalgono Sesle- ria argentea (Savi) Savi, Luzula nivea (Nathh.) DC., Hepatica nobilis Mill. e Primula vulgaris L. subsp. vulgaris (Gentile, 1975; Castelli et al., 2001; Andreis \& Sartori, 2011).

Sia nel piano montano che in quello collinare, le formazioni forestali di origine naturale si alternano ad impianti di conifere, realizzati, a partire dagli anni '30 del secolo scorso e con maggiore impegno negli anni '50 e '60, dal Corpo Forestale dello Stato, con lo scopo di consolidare le aree idrogeologicamente più dissestate. Vennero utilizzate svariate specie di conifere, quasi tutte estranee alla flora locale, come Pinus nigra J.F.Arnold subsp. nigra, Pinus sylvestris L. (di cui forse esistevano popolazioni autoctone in tempi antichi), Abies alba Mill. e Larix decidua Mill., talora accompagnate da Fraxinus ornus subsp. ornus e F. excelsior L. Alle quote inferiori gli interventi diedero risultati mediocri, sia per la natura dei suoli, sia per la difficile acclimatazione delle specie impiegate; in montagna, al contrario, gli impianti si rivelarono un successo (come ad esempio nell'area dell'attuale SIC IT2080021 "Monte Alpe"), grazie soprattutto alle capacità colonizzatrici di Pinus nigra (Guzzi, 2004), che ne hanno promosso una rapida espansione anche nelle fitocenosi naturali. Il substrato di questi impianti, fortemente acidificato dalle foglie delle conifere, è floristicamente molto povero; non mancano tuttavia specie interessanti, come Goodyera repens (L.) R.Br., orchidea introdotta attraverso le specie arboree utilizzate per la riforestazione (Romani \& Alessandrini, 2001).

Il disboscamento della faggeta, condotto sin dai tempi più remoti per favorire il pascolo, ha determinato nel tempo lo sviluppo di praterie secondarie mesoxerofile sulle vette e sui crinali più elevati, a quote superiori i 1.300 m (Castelli et al., 2001) (Fig. 10). Queste formazioni, attribuite all'alleanza Bromion erecti, sono contraddistinte 


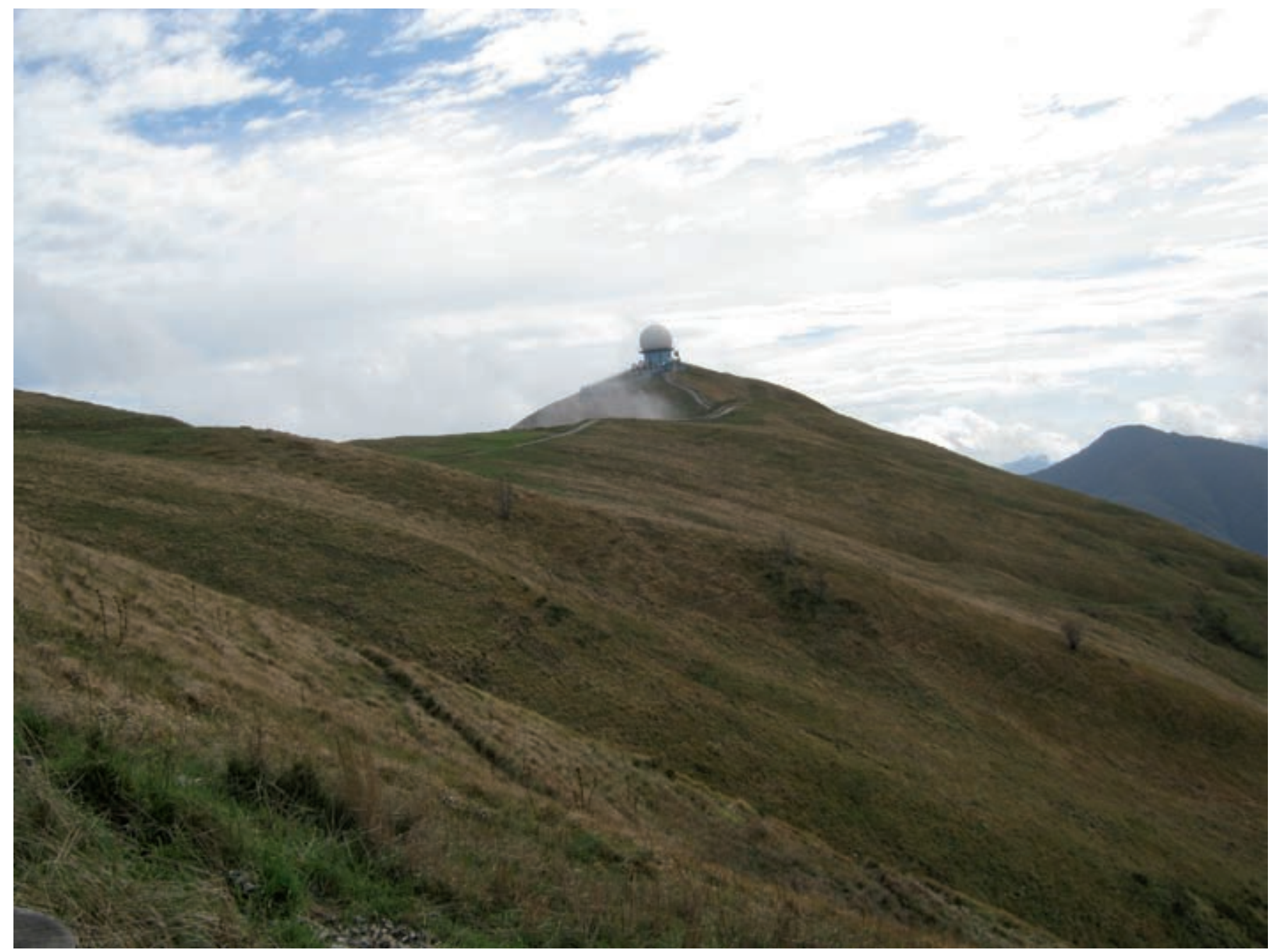

Fig. 10 - Praterie montane mesoxerofile sulla vetta del Monte Lesima (Foto: Nicola M.G. Ardenghi).

da un'elevata diversità floristica, dovuta a un intreccio di fattori fisici (esposizione, altitudine, reazione del suolo) e gestionali (pascolo bovino). Tra i taxa più frequenti si ricordano Festuca laevigata Gaudin, Brachypodium genuense (DC.) Roem. \& Schult., Hypericum richeri Vill. subsp. richeri, Serratula tinctoria L. subsp. monticola (Boreau) Berher, Leucanthemum heterophyllum (Willd.) DC. e Knautia drymeia Heuff. subsp. intermedia (Pernh. \& Wettst.) Ehrend., che dominano in stazioni pianeggianti sulla sommità dei crinali; sui versanti più acclivi, invece, aumenta la copertura di Sesleria pichiana Foggi, Gr.Rossi \& Pignotti, Phyteuma italicum Arv.-Touv., Siler montanum Crantz subsp. montanum e Plantago argentea Chaix subsp. argentea. In condizioni di maggiore acidità del substrato, dovuta al pascolo più intenso e alla liscivazione, compaiono, talora in modo preponderante, Festuca rubra subsp. juncea, Nardus stricta L., Avenella flexuosa (L.) Drejer subsp. flexuosa, Luzula campestris (L.) DC., Gentiana acaulis L., Arnica montana L. subsp. montana e specie spinose come Cirsium acaulon (L.) Scop. subsp. acaulon e C. eriophorum (L.) Scop. s.l. (Barcella et al., 2014). Così come gli xerobrometi di collina, anche le praterie montane sono interessate dalla presenza di orchidee [alcune delle quali molto rare, come Nigritella rhellicani Teppner \& E.Klein e Dactylorhiza viridis (L.) R.M.Bateman, Pridgeon \& M.W.Chase] e quindi riconducibili all'habitat prioritario $6210\left({ }^{*}\right)$, recentemente identificato, assieme ad altri habitat dell'allegato I della dir. n. 92/43/CEE (Direttiva "Habitat"), nell'area della Riserva naturale "Le Torraie-Monte Lesima", recentemente istituita (d.g.r. Lombardia 22 febbraio 2016, n. X/4838). A partire dal secondo dopoguerra lo spopolamento della montagna e la riduzione delle attività agro-pastorali (Maffi, 2010), dalle quali dipende la sopravvivenza di gran parte delle praterie culminali, hanno riattivato i naturali processi dinamici della vegetazione, con la progressiva ricolonizzazione da parte degli arbusti precursori della faggeta (Castelli et al., 2001). Nonostante l'abbandono dei centri montani, l'antropizzazione alle quote più elevate è proseguita, concentrandosi in particolare sulle vette più imponenti, che rappresentano siti strategici sin dall'antichità (secondo la leggenda, nel 218 a.C. Annibale, prima o dopo la battaglia del Trebbia, si recò in cima al Monte Lesima per meglio scrutare le valli sottostanti; proprio in quell'occasione cadde e si ferì una mano, pronunciando con ira l'espressione "laesit manus", da cui il nome del 
monte: Merlo, 1979). Dalla fine della Seconda guerra mondiale qui sono sorti vistosi impianti di radiocomunicazione, come il trasmettitore radiotelevisivo del Monte Penice (uno dei più importanti d'Italia, è stato costruito tra il 1952 e il 1953: Bertolotti, 1954) e il radar aeronautico del Monte Lesima (ultimato nel 1990: Gobbi, 2004).

In prossimità delle creste montuose si rinvengono gli unici, sporadici esempi di comunità vegetali riconducibili al piano subalpino. Si tratta di arbusteti bassi o prostrati, costituiti in prevalenza da Vaccinium myrtillus L., V. gaultherioides Bigelow, Juniperus communis L. subsp. alpina (Suter) Celak. e Rosa pendulina L. (Rhododendro ferruginei-Vaccinion myrtilli A.Schnyd. 1930), che si sviluppano sui crinali ad esposizione nord e con substrato acidificato per effetto della liscivazione; sui versanti ad esposizione sud, caratterizzati da suolo basico, prevalgono invece gli arbusteti a Cytisanthus radiatus (L.) O.Lang.

Gli affioramenti di serpentino delle alte Valli Staffora e Tidone sono contraddistinti da una flora peculiare, frutto della forte pressione selettiva esercitata dai suoli che caratterizzano questi luoghi, ricchi in metalli pesanti, scarsamente fertili ed estremamente aridi. La copertura vegetale è discontinua e costituita in prevalenza da camefite serpentinicole (Alyssion bertolonii Pignatti in E.Pignatti \& Pignatti 1977) (Biondi, 2000; Bogliani et al., 2003; Biondi \& Blasi, 2015), alcune delle quali endemiche, come Odontarrhaena argentea (All.) Ledeb. (Fig. 11B) e Cherleria laricifolia (L.) Iamonico subsp. ophiolitica (Pignatti) Iamonico (Fig. 11C). A queste si associano Linum campanulatum L., Asplenium adulterinum Milde subsp. adulterinum, $A$. cuneifolium Viv. subsp. cuneifolium, $\mathrm{Pa}$ ragymnopteris marantae (L.) K.H.Shing, Centaurea stoebe L. subsp. australis (A.Kern.) Greuter, Festuca stricta Host subsp. trachyphylla (Hack.) Patzke ex Pils (vicariante F. robustifolia Markgr.-Dann.), Robertia taraxacoides (Loisel.) DC., Cerastium arvense L. subsp. strictum (W.D.J.Koch) Gremli e subsp. suffruticosum (L.) Ces., Armeria arenaria (Pers.) Schult. subsp. arenaria e Fritillaria montana Hoppe ex W.D.J.Koch, quest'ultima, in Lombardia, esclusiva del Monte Pan Perduto. Gli affioramenti serpentinitici dell'alta Val Tidone (Monti Pan Perduto e Pietra di Corvo, località Sassi Neri) (Fig. 11) fanno parte di un'ampia area, denominata "Sassi Neri-Pietra Corva", recentemente candidata quale sito di importanza comunitaria (d.g.p. Provincia di Pavia 27 maggio 2014, n. 188/32965). Alle pendici del Monte Pietra di Corvo sorge il "Giardino Alpino di Pietra Corva" (Romagnese), fondato nel 1967 da Antonio Ridella; grazie alla sua posizione e alle sue collezioni viventi, ha da sempre svolto un importante ruolo didattico-educativo (Bogliani et al., 2003).

Nei pressi dell'abitato di Feligara (Brallo di Pregola) è localizzata una delle pochissime aree umide dell'Oltrepò montano: si tratta di una piccola torbiera bassa alcalina (Caricion davallianae Klika 1934), la cui esistenza è messa a repentaglio dal progressivo disseccamento registratosi negli ultimi anni. Al suo interno crescono alcune delle specie più rare dell'Oltrepò Pavese, come Epipactis palustris (L.) Crantz, Parnassia palustris L. subsp. palustris ed Eriophorum latifolium Hoppe; le ultime due sono presenti anche in un'altra zona umida, ubicata in corrispondenza della Sorgente Fontanassa in località Sassi Ne- ri (Romagnese). A poca distanza da quest'ultima, in località Gabiàs, è presente uno stagno di modeste dimensioni, sopravvissuto ad un tentativo di bonifica avvenuto una decina di anni fa; qui risiede l'unica popolazione oltrepadana di Eleocharis palustris (L.) Roem. \& Schult. subsp. palustris accertata in tempi recenti (Fig. 5B).

La vegetazione riparia dei maggiori torrenti appenninici (Staffora e Tidone) è rappresentata da comunità pioniere arboreo-arbustive con dominanza di salici [Salix eleagnos Scop. subsp. eleagnos, S. apennina A.K.Skvortsov, S. triandra L., Populus nigra L., Hippophaë fluviatilis (Soest) Rivas Mart.] (Salicion incanae Aichinger 1933), che si sviluppano sui greti ciottolosi e ghiaiosi (Biondi \& Blasi, 2015); anticamente in questi ambienti cresceva Myricaria germanica (L.) Desv., non più ritrovata dalla prima metà del Diciannovesimo secolo.

\section{Storia della ricerca floristica in Oltrepò Pavese}

Nella seconda metà del Diciottesimo secolo il territorio compreso nell'attuale provincia di Pavia appariva così suddiviso: il Pavese (a est del Ticino), assieme ad altri comuni ora appartenenti alla provincia di Milano (es. Abbiategrasso, Albairate, Besate, Binasco, Gaggiano, Magenta, Motta Visconti, Rosate), costituiva la provincia di Pavia della Lombardia austriaca, mentre la Lomellina e l'Oltrepò Pavese (includente Bobbio) facevano parte del Regno di Sardegna (Regione Lombardia, 2002-2015). Non è quindi un caso che $\mathrm{i}$ primi dati floristici relativi all'Oltrepò Pavese siano stati pubblicati da un botanico piemontese, Carlo Allioni (1728-1804), il quale, nella sua Flora pedemontana (Allioni, 1785), riporta dieci specie per la Valle Staffora, quattro delle quali [tra cui Ruta montana (L.) L. e Bombycilaena erecta (L.) Smoljan., non più ritrovate in tempi recenti] comunicategli da Ignazio Molineri (1741-1818). Negli stessi anni a Pavia la botanica iniziava ad assumere un ruolo sempre più prominente, favorita dall'azione riformatrice dell'imperatore austriaco Giuseppe II: nel dicembre 1763 fu istituita la prima cattedra di botanica dell'Università di Pavia, sin da subito ricoperta dal padre vallombrosano oriundo bavarese Fulgenzio Vitman (1728-1806), fondatore nel 1773 dell'attuale Orto botanico di Pavia (Visconti 2012a, 2012b, 2015). Vitman fu il capostipite di una lunga serie di direttori e professori di botanica (non di rado scandita da conflittualità personali) che determinarono, nel corso dei secoli, destini alterni per la botanica pavese, con evidenti ricadute anche sulla storia della floristica locale.

L'abate dell'Ordine dei Frati Predicatori Domenico Nocca (1758-1841), nativo di Giovenzano (Vellezzo Bellini, Pavia), fu il quarto direttore dell'orto botanico pavese, subentrando in qualità di supplente a Giovanni Antonio Scopoli, morto nel 1788; nel 1797 ottenne la cattedra di botanica, che mantenne fino al 1826 (Saccardo, 1895; Pollacci, 1936; Ciferri, 1961). "Uomo estroso ed irrequieto, di vivace ingegno ma polemico e litigioso" (Ciferri, 1961), Nocca nel 1816 ebbe il merito di pubblicare la prima flora interamente dedicata alla provincia di Pavia, Flora ticinensis (Nocca \& Balbis, 1816, 1821), la cui area d'indagine spaziava fino al Colle di San Colombano (all'epoca parte della provincia di Lodi e Crema del Regno Lombardo-Veneto). Per la realizzazione dell'opera 
A
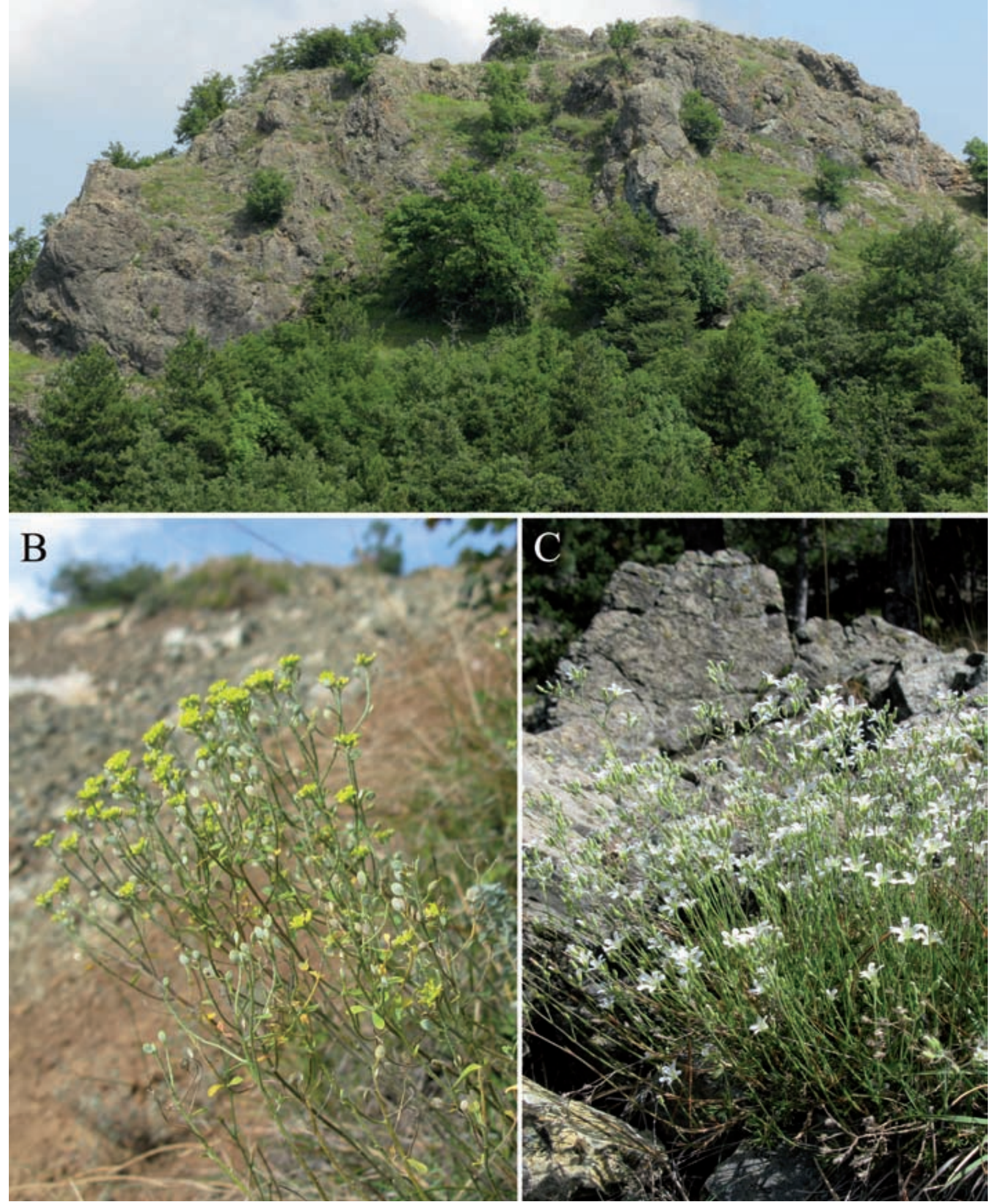

Fig. 11 - I serpentini del Monte Pietra di Corvo (Romagnese): A) vetta del monte (vista dal Monte Pan Perduto); B) Odontarrhaena argentea (All.) Ledeb.; C) Cherleria laricifolia (L.) Iamonico subsp. ophiolitica (Pignatti) Iamonico (Foto: Francesco Polani). 
si avvalse della collaborazione del piemontese Giovanni Battista Balbis (1765-1831), ritiratosi a vita privata dopo essere stato espulso nel 1814 dall'Orto botanico di Torino (che dirigeva) con il ritorno di Vittorio Emanuele I, di cui era strenuo oppositore (Colla, 1833; Moretti, 1851; Università di Torino, Orto Botanico, 1929). Tra i taxa descritti in Flora ticinensis si ricorda Carduus litigiosus Nocca \& Balb., con locus classicus rappresentato da diverse località della Valle Staffora, tra cui Pregola, dove la specie cresce tuttora. Nello stesso periodo l'Oltrepò montano venne visitato dal botanico pavese Giuseppe Bergamaschi (1785-1867), che trascrisse le cronache dei suoi viaggi in due lettere pubblicate (Bergamaschi, 1823a, 1823b, 1824a, 1824b), da cui traspaiono le difficoltà dell'epoca nello spostamento tra Pavia e i rilievi appenninici. I campioni di Nocca e Bergamaschi, unitamente a quelli di un altro botanico pavese molto attivo in quegli anni, il medico Francesco Bonfico (cfr. Moretti, 1851; Garovaglio, 1862; Ardenghi, 2013b), sono tuttora conservati presso l'erbario dell'Università di Pavia (PAV); come riferito da Maffei (1921), negli anni a cavallo tra Ottocento e Novecento questi exsiccata, che giacevano "abbandonati alla rinfusa" presso l'ex Orto agrario di Pavia, vennero trasferiti all'ex Museo Civico di Storia Naturale della città, annesso all'Istituto Tecnico Agronomico “A. Bordoni" di Via Mascheroni (oggi Istituto Tecnico Commerciale Turistico, in Via S. Carlo: Sistema Museale di Ateneo, 2015). Angelo Carabelli, conservatore del museo, riuscì a riordinare i campioni sopravvissuti all'incuria e costituì l'Erbario Pavese, successivamente intercalato nell'Erbario Lombardo (Ciferri, 1951) con la dicitura "Ist. Bordoni - Pavia, Erbario Pavese" timbrata lungo il margine superiore dei fogli d'erbario (Ardenghi, 2013b).

Dopo un anno trascorso come direttore dell'Orto agrario di Pavia (1814), nel 1826 Giuseppe Moretti (17821853), originario di Roncaro (Pavia), succedette a Domenico Nocca nella direzione dell'orto botanico, criticando sin da subito l'operato del suo predecessore (Bianchi \& Pozzi, 1959). Sotto la sua prefettura (conclusasi nel 1852), la botanica pavese conobbe uno dei periodi di massimo splendore. Moretti, durante la sua carriera, si dimostrò infatti uno scienziato eclettico, occupandosi di botanica sistematica, agraria e chimica (Bianchi et al., 1959). Tra le specie da lui descritte per la provincia di Pavia si ricordano Xanthium italicum Moretti e Cnicus spathulatus Moretti [三Cirsium eriophorum subsp. spathulatum (Moretti) Ces.], quest'ultima con locus classicus nei colli "ad meridiem Papiae". Non scrisse alcuna opera sulla flora pavese, ma contribuì ad istruire validissimi studenti, come il lecchese Lorenzo Rota (1818-1855) e il lodigiano Filippo Morandini (18261903) (Ardenghi, 2013c). Rota, in particolare, si rivelò una figura chiave nella floristica pavese: nel 1847 pubblicò il Prospetto delle Piante fanerogame ritrovate nella Provincia Pavese (Rota, 1847), minuzioso aggiornamento di Flora ticinensis e ultima flora completa della provincia di Pavia (Ardenghi, 2015a, 2015b). Per la stesura dell'opera, il medico di Carenno realizzò numerosi campioni d'erbario, oggi conservati in PAV e in BER (Rinaldi, 2009); così come fece Antonio Bertoloni per la sua Flora itali$c a$, acquisì dati di campo dai milanesi Giuseppe Balsamo Crivelli (1800-1874) e Giuseppe De Notaris (1805-1877), che ebbero occasione di percorrere l'Appennino Pavese e Piacentino presumibilmente durante il loro periodo di studi a Pavia. Rota ricevette una grande quantità di informazioni anche da un altro botanico di Milano, il barone Vincenzo de Cesati (1806-1883), che nel 1835 compì una fruttuosa spedizione attraverso tutto l'Oltrepò Pavese, spingendosi a sud fino a Torriglia (Genova); di quel viaggio rimangono tre blocchi di appunti, attualmente depositati presso l'Archivio di Stato di Vercelli, Fondo Arborio Mella (Cesati, 1835a, $1835 \mathrm{~b}, 1835 \mathrm{c}$; il secondo di questi è privo di località, salvo che per due specie), e svariati campioni d'erbario conservati in RO-Cesati (Soldano, 1983). Un altro studente di Moretti fu lo stradellino Giovanni Belli (1812-1904), figlio del matematico e fisico ossolano Giuseppe (1791-1860), i cui campioni oltrepadani, raccolti attorno agli anni '30 dell'Ottocento, costituiscono una collezione a parte in PAV.

Le perlustrazioni floristiche in Oltrepò Pavese proseguirono per tutta la seconda metà dell'Ottocento, protraendosi fino al primo decennio del secolo successivo. Protagonisti di questo periodo furono numerosi botanici gravitanti attorno alla figura del ferrarese Giovanni Briosi (1846-1919), direttore, tra il 1883 e il 1919, dell'Istituto Botanico e del Laboratorio Crittogamico, istituiti presso l'orto botanico sotto la fulgida direzione di Santo Garovaglio (1805-1882), tra il 1853 e il 1882 (Pirola, 1992). Rodolfo Farneti (1859-1919), Fridiano Cavara (1857-1929), Pasquale Baccarini (1858-1919), Giovanni Luigi Pavarino (1867-1937), Giovanni Battista Traverso (1878-1955; da non confondere con Giacomo, giardiniere dal 1877), Siro Luigi Maffei (1879-?), Vittorio Pavesi (?-?), Luigi Bozzi (?-?) e Achille Lodi (?-?) furono figure di passaggio negli istituti di Pavia (con l'eccezione di Farneti e Maffei), dove ricoprirono principalmente il ruolo di assistente (Saccardo, 1895, 1901; Briosi, 1914; Pollacci, 1918; Govi, 1995; Università degli Studi di Pavia, 2005-2015; Ardenghi, 2013b). Benché abbiano svolto attività di ricerca soprattutto nell'ambito della micologia e della fitopatologia, durante gli anni della loro formazione scientifica diedero un contributo determinante alla ricerca floristica pavese, espressamente incoraggiata da Briosi, che individuò nel bolognese Farneti il coordinatore delle attività di raccolta e di revisione dei campioni d'erbario (Briosi in Farneti, 1900). I numerosi reperti raccolti in questo periodo andarono a costituire l'Herbarium o Collectio Agri Ticinensis, istituito da Briosi nel 1884 (Pollacci, 1918) e successivamente intercalato nell'Erbario Lombardo, di cui oggi è parte integrante (gli exsiccata oltrepadani di Pavesi, a differenza di quelli raccolti nei dintorni di Pavia, non appartengono a questa collezione, ma sono depositati in MSPC; cfr. Pavan Arcidiaco et al., 1982, e Romani \& Alessandrini, 2001).

Le vivaci attività esplorative condotte in Oltrepò Pavese tra Ottocento e primo decennio del Novecento, per nulla scoraggiate dalle difficoltà logistiche dell'epoca, consentirono di mantenere costante la pubblicazione di dati floristici, che trovarono spazio non solo all'interno delle due flore di Nocca \& Balbis $(1816,1821)$ e Rota $(1847)$, ma anche in un buon numero di articoli a carattere strettamente floristico, rappresentati da aggiornamenti alla flora provinciale (es. Farneti, 1900; Pollacci, 1918; Maffei, 1921) e da florule tematiche incentrate sull'Appennino (es. Pavesi, 1906; Pavarino, 1914, 1915) (Fig. 12). 
Con la fine della direzione di Briosi (1919), l'esplorazione floristica in Oltrepò Pavese e più in generale nella provincia di Pavia subì un forte contraccolpo; nuove opere floristiche dedicate, almeno parzialmente, a questo territorio vedranno la luce solo negli anni '50 dello stesso secolo (Fig. 12). Il "medioevo floristico" pavese affonda le sue radici nelle scelte operate in ambito scientifico dai successori di Briosi, Luigi Montemartini (1869-1952) di Montù Beccaria e Gino Pollacci (1872-1963) di Pavia, che indirizzarono la ricerca sempre più verso la patologia vegetale, la micologia e la botanica farmaceutica (Ciferri, 1961, 1964). Giocarono un ruolo decisivo anche le travagliate vicende storiche e politiche dell'epoca: dapprima il regime fascista ostacolò sul piano professionale Montemartini, deputato socialista, che alla fine del 1926 venne sospeso dalla cattedra universitaria e condannato a due anni di confino a Roma (Ciferri, 1961; Scagni, 2009; Sircana, 2012). In secondo luogo la guerra e ancor di più la Repubblica di Salò contribuirono a trasformare l'Oltrepò Pavese in un teatro di scontri ed efferate rappresaglie, perpetuate dai nazi-fascisti soprattutto sui rilievi appenninici dove le formazioni partigiane trovavano rifugio (Scagni, 2003). L'unico botanico che in quegli anni ebbe il coraggio di avventurarsi tra i colli oltrepadani fu Luigi Ceroni (1883-1951), medico bolognese operante dal 1932 all'Ospedale psichiatrico di Como. Almeno dai primi anni '20 trascorse periodi di vacanza in località Malaspina di Canneto Pavese (Ardenghi, 2014), campo base per le sue escursioni in Valle Versa e dintorni, che proseguirono in modo piuttosto costante fino al 1948. Alla sua scomparsa (avvenuta proprio a Malaspina), Ceroni lasciò in eredità una notevole collezione di piante essiccate ("oltre trecento pacchi"), che la vedova donò all'Erbario di Pavia attorno al 1952 (Giacomini, 1952), dove vennero intercalate nell'Erbario Lombardo; tra queste figurano oltre 400 campioni raccolti in Oltrepò Pavese (Ardenghi, 2014).

Con la fine della guerra, Raffaele Ciferri (1897-1964), che era subentrato nel 1942 a Pollacci in qualità di direttore dell'orto botanico, fece ritorno a Pavia; avendo solidarizzato con i movimenti di resistenza, fino ad allora rimase nascosto nelle Langhe per sfuggire alle rappresaglie tedesche (Giacomini, 1981). Con Ciferri la botanica pavese riprese vita: nei primi anni '50 egli riorganizzò le collezioni erbariologiche, istituendo l'Erbario Lombardo (Ciferri, 1951); si circondò di nuovi collaboratori, tra cui Sandro Pignatti (1930-vivente), che in quegli anni contribuì alla sistemazione dell'erbario e ad arricchire lo stesso con nuovi reperti anche oltrepadani. Nello stesso periodo andava affermandosi la fitosociologia, che fino alla fine del secolo ricoprirà a Pavia un ruolo dominante. L'Oltrepò Pavese sin da subito si trasformò in una palestra per gli studiosi di vegetazione, che pubblicarono nel corso dei decenni svariati contributi sulle comunità forestali (Gentile, 1975; Ubaldi et al., 1993; Andreis \& Sartori, 2011), sulla vegetazione delle colture cerealicole (Pignatti, 1957), dei calanchi (Volk, 1958; Gentile \& Sartori, 1975) e degli affioramenti di serpentino (Pignatti, 1967). È in queste opere dove si rileva una consistente parte delle informazioni floristiche prodotte tra il 1951 e il 2000 (Fig. 12): in questo periodo, infatti, la floristica assunse un ruolo subalterno rispetto alla fitosociologia e i lavori dedicati, anche solo marginalmente, alla flora dell'Oltrepò Pavese furono pochissimi (es. Viola, 1953; Pirola, 1964; Soldano, 1980). Si affievolì anche il numero dei campioni oltrepadani intercalati nell'Erbario Lombardo, con un arresto pressoché totale nei primi anni '90 (dal 12 maggio 2012, con il pensionamento dell'ultimo tecnico, Maurizio Preti, le attività dell'erbario sono cessate).

In controtendenza con quanto avvenuto nell'ultimo secolo, tra il 2001 e il 2015 si è verificato un netto aumento nella produzione di dati floristici, con la pubblicazione di oltre 100 opere tra articoli e segnalazioni, molte delle quali relative a novità di rilevanza regionale e nazionale (es. Ardenghi \& Polani, 2010a, 2010b; Ardenghi, 2012, 2013a, 2013b); hanno inoltre visto la luce lavori di stampo tassonomico (es. Ardenghi et al., 2014), monografie floristiche di respiro regionale (es. Banfi \& Galasso, 2010) oppure dedicate a singoli gruppi sistematici (es. Abeli et al., 2012) (Fig. 12). Il vistoso miglioramento delle conoscenze si deve in particolare alla volontà degli scriventi di aggiornare il quadro floristico oltrepadano e al conseguente impegno degli stessi in un'esplorazione più puntuale ed omogenea del territorio, nello studio critico delle fonti bibliografiche e nella revisione dei campioni conservati presso l'Erbario Lombardo dell'Università di Pavia (al quale, nel 2011, è stata affiancata una nuova unità, l'Erbario di lavoro, tuttora in fase di allestimento; cfr. Rossi \& Ardenghi, 2015). Le informazioni scaturite da questa intensa attività di ricerca hanno permesso agli autori di impostare le basi per un'opera floristica di sintesi che mancava da oltre centocinquant'anni e che vede nel presente contributo la sua realizzazione.

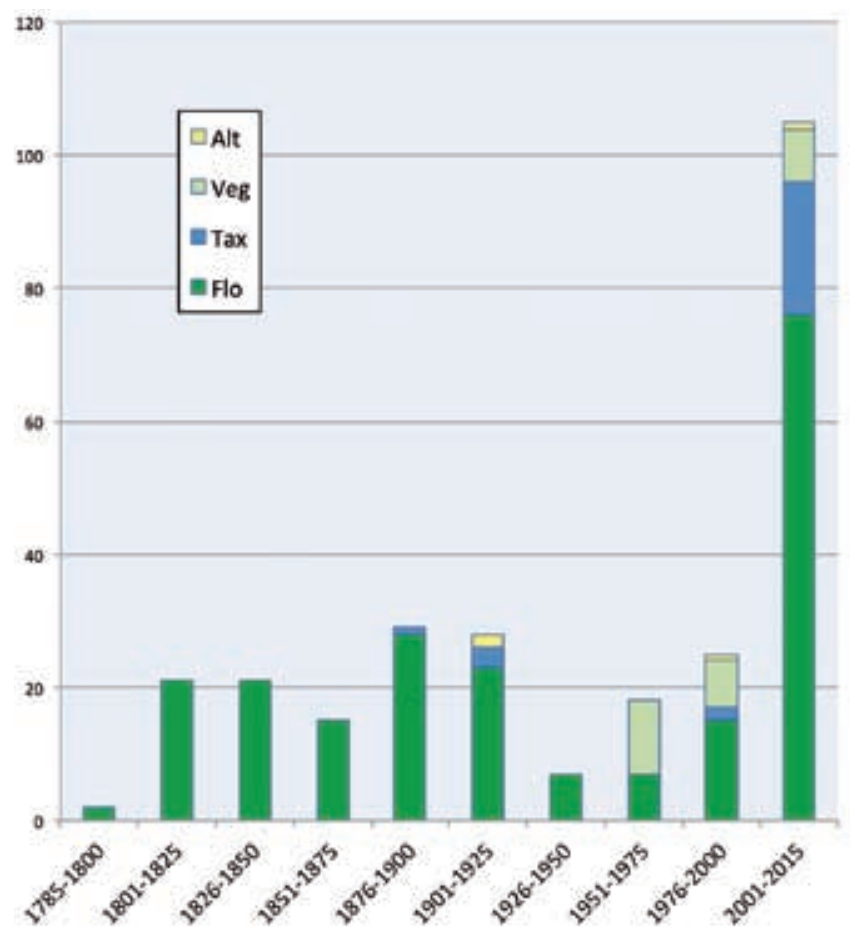

Fig. 12 - Pubblicazione di lavori floristici relativi all'Oltrepò Pavese tra il 1785 e il 2015, valutata su intervalli di 25 anni (con l'eccezione del primo e dell'ultimo). Sono state identificate quattro tipologie di fonti bibliografiche: floristica (Flo), tassonomica (Tax), vegetazionale (Veg), miscellanea (Alt). 


\section{MATERIALI E METODI}

La presente opera si compone di due elenchi floristici (Appendice 1), uno includente i taxa accettati ("Elenco floristico") e un altro ("Taxa dubia et excludenda") comprensivo dei taxa di presenza dubbia ("dubia") o segnalati per errore ("excludenda").

I dati utilizzati per la compilazione dei suddetti elenchi derivano in larga parte dalle indagini di campo condotte dagli autori in Oltrepò Pavese negli ultimi vent'anni; i campioni raccolti sono stati depositati presso l'erbario personale del primo autore (situato a Stradella), l'erbario del Museo Naturalistico "Ferruccio Lombardi" di Stradella, l'erbario del Museo Civico di Storia Naturale di Milano (MSNM) e l'Herbarium Centrale Italicum (FI) (acronimi secondo Thiers, 2014 onwards). Ulteriori informazioni sono state acquisite dalla consultazione critica di 271 fonti bibliografiche riguardanti, anche solo parzialmente, l'area di studio e dalla revisione di numerosi exsiccata conservati in diverse collezioni pubbliche e private (cfr. Appendice 1). Tutti i dati raccolti sono stati costantemente inseriti ed elaborati all'interno di un database "FileMaker Pro Advanced 11.0v2".

I taxa accettati sono elencati secondo l'ordine sistematico a livello di sottoclasse proposto da Christenhusz et al. (2011a, 2011b) e The Angiosperm Phylogeny Group (2016); famiglie, generi, specie e sottospecie sono ordinati alfabeticamente all'interno di ogni sottoclasse (sono stati omessi gli ordini per facilitare la consultazione alfabetica delle famiglie all'interno delle sottoclassi). L'elenco dei taxa dubia et excludenda è disposto in ordine alfabetico.

Per la delimitazione sistematica delle sottoclassi e delle famiglie si è fatto riferimento a Christenhusz et al. (2011b) e a Rothfels et al. (2012) per le "pteridofite", a Christenhusz et al. (2011a) per le "gimnosperme" e a The Angiosperm Phylogeny Group (2016) per le "angiosperme" [con l'eccezione delle Chenopodiaceae, mantenute separate dalle Amaranthaceae (cfr. Hernández-Ledesma et al., 2015), delle Dipsacaceae e delle Valerianaceae, non incluse nelle Caprifoliaceae (cfr. Backlund \& Pick, 1998; Cole et al., 2016), delle Heliotropiaceae e delle Hydrophyllaceae, non riunite nelle Boraginaceae (cfr. Cecchi \& Selvi, 2015a, 2015b), e delle Myrsinaceae, trattate come famiglia indipendente rispetto alle Primulaceae (cfr. Källersjö et al., 2000)].

Per ogni taxon dell'"Elenco floristico" vengono indicati: 1) nome attualmente accettato (la nomenclatura segue in massima parte Conti et al., 2005, 2007, e gli aggiornamenti più recenti in materia);2) sinonimi; 3 ) indigenato; le entità alloctone sono classificate in base al periodo d'introduzione (archeofita, neofita, amaurogena) (cfr. Banfi \& Galasso, 2010) e per ognuna di esse sono specificati lo status d'invasività (cfr. Celesti-Grapow et al. 2009, 2010) e l'eventuale appartenenza alla Lista Nera di cui alla 1.r. Lombardia n. 10/2008 (d.g.r. Lombardia 24 luglio 2008, n. 8/7736); sono classificate come "esotiche a livello locale" le entità autoctone a livello nazionale ma alloctone in Oltrepò Pavese; 4) areale d'origine, che si è preferito utilizzare al posto del "corotipo globale" sensu Fattorini (2015), tradizionalmente adottato dalle flore italiane in conformità con
Pignatti (1982); quest'ultimo concetto, riferendosi alla distribuzione mondiale di un taxon (quindi alla somma dell'areale primario e di quello secondario), avrebbe determinato la proliferazione di taxa a distribuzione subcosmopolita o cosmopolita, fuorviando il reale significato biogeografico della flora; viene inoltre specificato se il taxon è un endemita italiano (cfr. Peruzzi et al., 2014, 2014 onwards, 2015); 5) tipo biologico secondo Pignatti (1982); 6) status di conservazione: è riportata la categoria di minaccia secondo la Lista Rossa della Flora Italiana (Rossi et al., 2013) e l'eventuale appartenenza agli allegati II, IV e V della dir. n. 92/43/ CEE (Direttiva "Habitat") e agli elenchi di flora protetta $\mathrm{C} 1$ e C2 di cui alla 1.r. Lombardia n. 10/2008 (d.g.r. Lombardia 24 luglio 2008, n. 8/7736 e d.g.r. Lombardia 27 gennaio 2010, n. 8/11102); 7) habitat; 8) distribuzione altitudinale; 9) distribuzione geografica in Oltrepò Pavese; 10) frequenza; 11) osservazioni di campo; 12) campioni d'erbario esaminati; 13) bibliografia consultata; 14) note. Un taxon viene considerato non più ritrovato se il dato di presenza più recente è antecedente o risalente al 1980 oppure se dopo tale data è stata accertata la sua estinzione. Per i taxa dubbi o da escludere vengono riportati: 1) nome attualmente accettato; 3 ) famiglia di appartenenza; 4) sinonimi; 5) status (taxon di presenza dubbia o segnalato per errore); 6) bibliografia consultata; 7) note. Informazioni più dettagliate e simbologia adottata sono riportate nella legenda all'inizio dell'Appendice 1 .

La distribuzione altitudinale ricalca la suddivisione dell'area di studio nei tre piani altitudinali descritti nel paragrafo introduttivo "Il paesaggio vegetale": planiziale $(50-300 \mathrm{~m})$, collinare (301-900 m), montano (9011724 m) (Fig. 2).

Per valutare la distribuzione dei singoli taxa in Oltrepò Pavese sono stati individuati dieci settori, definiti in base a criteri di tipo fisico, geografico, vegetazionale e bioclimatico (Fig. 1):

(1) Pianura Padana: è la porzione meridionale della piana del Po, delimitata a sud dai primi rilievi collinari;

(2) bassa Valle Staffora: comprende la valle del torrente Staffora dal centro abitato di Retorbido a quello di Varzi, incluse le valli dei torrenti Rile, Ardivestra, Nizza, Lella (affluenti dello Staffora), Luria e Brignolo (quest'ultimo riceve il Luria prima di immettersi nel Po);

(3) Valle Coppa: comprende la valle del torrente Coppa a sud di Mezzadra di Montebello della Battaglia e dell'abitato di Casteggio oltre alle valli dei torrenti Ghiaia Coppa, Ghiaia di Montalto (entrambi confluiscono a Borgo Priolo per dare origine al Coppa), Rile di Casteggio, Schizzola (affluenti del Coppa) e Rio Fossagazzo (è noto con i nomi di "Lurietta Brignoli" e "Brignolino" a nord di Montebello della Battaglia e si immette nel torrente Brignolo a Lungavilla);

(4) bassa Val Tidone: corrisponde alla valle del torrente Tidone che si prolunga a sud di Pometo e del Bivio Carmine (Ruino), tra la porzione pavese del Lago di Trebecco e l'abitato di Romagnese;

(5) Valle Scuropasso: fanno parte di questo settore le valli del torrente Scuropasso (a sud fino a Pometo), del 
suo affluente Verzate e, da ovest verso est, le vallette del Rile San Zeno (affluente del Po), del Rile del Pizzolo (si immette nella Roggia Cappella tra Santa Giuletta e Barbianello, che a sua volta entra nel Verzate a Pinarolo Po) e la valle di Recoaro di Broni;

(6) Valle Versa: include tutta la valle del torrente Versa dalla porzione meridionale dell'abitato di Stradella fino alla SP59 in corrispondenza di Ca' Vannone di Ruino; appartiene a questo settore anche la valletta del Rio Fontanone o Sanguinolento (che si immette ad Arena Po, cambiando nome in Rio Pizzaretta, nello Scolo Rile, affluente del Po);

(7) Valle Bardonezza: corrisponde alla porzione della valle del torrente Bardonezza (o Bardoneggia) che si estende dal lato sud della frazione Cardazzo di Bosnasco fino a Pizzofreddo di Santa Maria della Versa; comprende anche la valle del Rio Marsinola, affluente del Bardonezza;

(8) alta Valle Staffora: include la valle del torrente Staffora tra Varzi e il crinale che la separa dalla Val Boreca piacentina, oltre alle valli laterali dei torrenti Reganzo e Aronchio; si estende a est fino ai crinali che dividono la Valle Staffora dalla Val Tidone pavese e dalla Val Trebbia piacentina;

(9) Valle Avagnone: incastonata nell'alta Valle Staffora, comprende la valle del torrente Avagnone (affluente del fiume Trebbia) a sud dell'abitato di Brallo di Pregola; è delimitata a ovest dalla SP88 e a est dalla SP166;

(10) alta Val Tidone: si estende a sud dell'abitato di Romagnese fino al crinale che separa la Val Tidone pavese dalla Val Trebbia piacentina.

Nella disamina delle fonti bibliografiche ed erbariologiche, i toponimi attualmente situati a cavallo tra la provincia di Pavia e quelle di Piacenza ed Alessandria (es. Monte Penice, Monte Lesima, Monte Chiappo) sono stati trattati come località pavesi salvo indicazioni esplicite sui versanti, sul comune o sulla provincia di appartenenza che provassero il contrario. Le segnalazioni per "Bobbio" antecedenti il 1923 fanno probabilmente riferimento non solo al comune di Bobbio (Piacenza) ma anche all'omonima ex provincia, comprensiva di alcuni territori attualmente appartenenti alla provincia di Pavia (cfr. il paragrafo introduttivo "Geografia"); nonostante questo, al fine di evitare disambiguità, tali segnalazioni non sono state prese in considerazione.

L'elenco dei toponimi citati negli elenchi floristici con i relativi settori di appartenenza è riportato in Appendice 2 .

La ricchezza floristica della provincia di Pavia, dei tre macrosettori provinciali e dei settori oltrepadani è stata ricavata secondo la metodologia esposta da Cristofolini (1998). Essa prevede il calcolo della formula di regressione lineare tra il logaritmo delle superfici delle aree considerate (in $\mathrm{km}^{2}$ ) e i logaritmi delle rispettive densità di taxa $\left(\operatorname{taxa} / \mathrm{km}^{2}\right)$. La retta risultante rappresenta le densità di taxa attese. Le densità di taxa osservate sono state infine rapportate con quelle attese, allo scopo di ottenere una misurazione oggettiva della ricchezza floristica, depurata dall'effetto "ampiezza dell'area" (la densità floristica tende infatti a diminuire in modo costante all'aumentare della superficie).

\section{RISULTATI}

Si vedano i due elenchi floristici in Appendice 1.

\section{DISCUSSIONE}

Sulla base degli elenchi floristici allegati al presente articolo (Appendice 1), la flora dell'Oltrepò Pavese annovera complessivamente 1.871 taxa (specie, sottospecie e ibridi) appartenenti a 739 generi e 138 famiglie. 183 entità, pari al $9,8 \%$ della flora, non sono state più ritrovate dopo il 1980; a queste se ne aggiungono 245 di presenza dubbia e 161 segnalate per errore (Tab. 1). Il numero dei taxa censiti in Oltrepò Pavese è pari al $48,4 \%$ della flora lombarda, che ammonta a 3.869 entità (esclusi gli ibridi; cfr. Galasso, 2015a). Prendendo in considerazione la suddivisione della provincia di Pavia in tre macrosettori (Lomellina, Pavese, Oltrepò Pavese; si veda il capitolo introduttivo), quello oltrepadano appare il più ricco, ospitando $1 ' 82,3 \%$ della flora provinciale; in quest'area, inoltre, il numero osservato di taxa è nettamente superiore rispetto a quello atteso in base all'estensione della sua superficie. La percentuale di taxa non più ritrovati dopo il $1980(9,8 \%)$ è nettamente inferiore rispetto a quelle di Lomellina $(29,6 \%)$ e Pavese $(26,1 \%)$, macrosettori interessati da un impatto antropico più aggressivo dovuto in larga parte alla risicoltura intensiva (Tab. 2).

Nell'ultima flora della provincia di Pavia (Rota, 1847: 289-292), l'Oltrepò Pavese contava 1.079 specie, derivanti dalla somma delle 673 specie "comuni ai due terreni Insubrico ed Oltrepadano" (con "Insubrico" Rota intende la porzione di provincia a nord del Po) alle 406 "speciali al terreno Oltrepadano". Non tenendo conto delle "pteridofite" (37 taxa), che Rota non contempla nel suo repertorio, rispetto a quest'ultima opera si registra un incremento di taxa pari al $70 \%$. Il confronto, tuttavia, è da ritenere puramente indicativo, in quanto Rota non considera nel conteggio totale $\mathrm{i}$ taxa infraspecifici (varietà) e le specie "avventizie" e "coltivate all'aperto, o già coltivate e fatte spontanee"; inoltre, dal 1847 ad oggi, la concezione sistematica di alcuni taxa non è più la stessa e i confini amministrativi dell'Oltrepò Pavese sono in parte mutati.

Tab. 1 - Prospetto numerico della flora dell'Oltrepò Pavese. $+=$ taxa accertati dopo il 1980; $0=$ taxa non più ritrovati dopo il 1980.

\begin{tabular}{|l|r|r|}
\hline & \multicolumn{1}{|c|}{ Tot } & \multicolumn{1}{|c|}{$\mathbf{\%}$} \\
\hline Totale (+, 0) & $\mathbf{1 . 8 7 1}$ & $\mathbf{1 0 0 , 0}$ \\
\hline Presenti (+) & 1.688 & 90,2 \\
\hline Non più ritrovati (0) & 183 & 9,8 \\
\hline Presenza dubbia (?) & 245 & - \\
\hline Segnalati per errore (-) & 161 & - \\
\hline
\end{tabular}


Tab. 2 - Prospetto numerico della flora della provincia di Pavia. $\mathrm{Km}^{2}=$ superficie (approssimata al $\mathrm{km}$ ); Taxa $=$ totale dei taxa censiti; $\% \mathrm{PV}=$ percentuale dei taxa in ogni macrosettore rispetto al totale della flora proviciale; Densità floristica $=$ densità floristica (taxa $/ \mathrm{km}^{2} ; \mathrm{cfr}$. Cristofolini, 1998) osservata (oss), attesa (att) e rapporto tra osservata e attesa (oss/att) per ogni macrosettore (in grassetto $\mathrm{i}$ valori $\geq 1$ ); $+=$ taxa accertati dopo il $1980 ; 0=$ taxa non più ritrovati dopo il $1980 ; \% 0=$ percentuale dei taxa non più ritrovati in ogni macrosettore e in provincia. Il rapporto tra densità floristica osservata e attesa relativo alla provincia di Pavia è stato ottenuto considerando le flore delle seguenti aree lombarde: province di Bergamo e Brescia (Bona, 2015), Como e Lecco (Banti et al., 2015), Cremona (Giordana, 2010a), Lodi (Giordana, 2010b), Mantova (Truzzi, 2015), Sondrio (Piazza, 2015) e Varese (Macchi, 2005), città di Milano (Banfi \& Galasso, 1998), Parco Agricolo Sud Milano (Brusa \& Rovelli, 2010).

\begin{tabular}{|c|c|c|c|c|c|c|c|c|c|}
\hline \multirow{2}{*}{ Macrosettore } & \multirow{2}{*}{$\mathrm{Km}^{2}$} & \multirow{2}{*}{ Taxa } & \multirow{2}{*}{$\% \mathrm{PV}$} & \multicolumn{3}{|c|}{ Densità floristica } & \multirow{2}{*}{+} & \multirow{2}{*}{0} & \multirow{2}{*}{$\% 0$} \\
\hline & & & & oss & att & oss/att & & & \\
\hline Lomellina & 1.274 & 1.270 & 55,9 & 0,997 & 1,194 & 0,835 & 894 & 376 & 29,6 \\
\hline Pavese & 595 & 1.341 & 59,0 & 2,254 & 2,353 & 0,958 & 991 & 350 & 26,1 \\
\hline Oltrepò Pavese & 1.100 & 1.871 & 82,3 & 1,701 & 1,361 & 1,250 & 1.688 & 183 & 9,8 \\
\hline Provincia di Pavia & 2.969 & 2.273 & - & 0,766 & 0,671 & 1,140 & 1.936 & 337 & 14,8 \\
\hline
\end{tabular}

Da un punto di vista numerico, la flora dell'Oltrepò Pavese occupa una posizione intermedia rispetto a quelle dei territori limitrofi: il numero totale di taxa supera del 9,9\% il numero di specie (1.702) indicato per il Tortonese e il Novese (porzione sud-orientale della provincia di Alessandria), la cui superficie, pari a circa $1.350 \mathrm{~km}^{2}$, è maggiore di quella oltrepadana (Carrega \& Silla, 1995, 1999); risulta invece inferiore del $9,3 \%$ rispetto al numero di taxa censiti in provincia di Piacenza, 2.063 (Bracchi \& Romani, 2010; per il calcolo di questo numero sono state considerate le entità autoctone e alloctone accertate e scomparse contrassegnate con le sigle "PA", "AN", "AC", "AR", "SC" nella Tab. 1 di pg. 201 e quelle elencate nell'appendice a pg. 393), distribuiti su un territorio più esteso $\left(2.589 \mathrm{~km}^{2}\right)$ e caratterizzato da rilievi montani più elevati (Romani \& Alessandrini, 2001).

Sulla base della regressione lineare calcolata per l'Oltrepò Pavese (Fig. 13), i tre settori montani (alta Val Tidone, alta Valle Staffora, Valle Avagnone), la Valle Versa, la Pianura Padana e la bassa Valle Staffora risultano i più ricchi dal punto di vista floristico, con un numero osservato di taxa superiore rispetto a quello atteso in base all'estensione in superficie (Tab. 3). Mentre i valori dei settori montani e della bassa Valle Staffora appaiono legati alla diversità ambientale che li caratterizza, quelli relativi alla Valle Versa e alla Pianura Padana si devono alla maggiore esplorazione floristica e al continuo ingresso di taxa esotici che contribuisce a mantenere elevato il numero complessivo di unità.

Prendendo in considerazione $\mathrm{i}$ taxa non più ritrovati negli ultimi trentasei anni, si nota che la maggior parte di questi cresceva in Pianura Padana $(42,6 \%)$, in alta $(27,9 \%)$ e in bassa Valle Staffora (21,9\%) (Tab. 3); tra le entità non più confermate figurano soprattutto piante di ambienti umidi [es. Osmunda regalis L., Nymphoides peltata, Nymphaea alba L., Typha minima Funck, Trapa natans L., Pulicaria vulgaris L., Myricaria germanica], distrutti o fortemente alterati nell'ultimo secolo, com- mensali dei campi coltivati [es. Lolium temulentum L., $L$. remotum Schrank, Bromus secalinus L. subsp. secalinus, Legousia hybrida (L.) Chaix., Turgenia latifolia (L.) Hoffm., Asperula arvensis L.] e specie d'alta quota; queste ultime erano anticamente diffuse soprattutto sulla vetta del Monte Penice [es. Erigeron alpinus L., Crepis aurea (L.) Cass. subsp. aurea, Dianthus superbus L. subsp. superbus, Lycopodium clavatum L., Luzula lutea (All.) DC. subsp. lutea, Potentilla brauneana Hoppe], dove l'impatto antropico e le attività turistico-ricreative del secondo dopoguerra hanno probabilmente compromesso la loro esistenza. Esaminando la flora dei singoli settori, la Valle Versa detiene la percentuale più elevata di taxa non più osservati in tempi recenti $(18,6 \%)$ : si tratta in prevalenza di entità legate ad ambienti naturali come praterie xerofile e querco-ostrieti, annientati dall'avanzare della

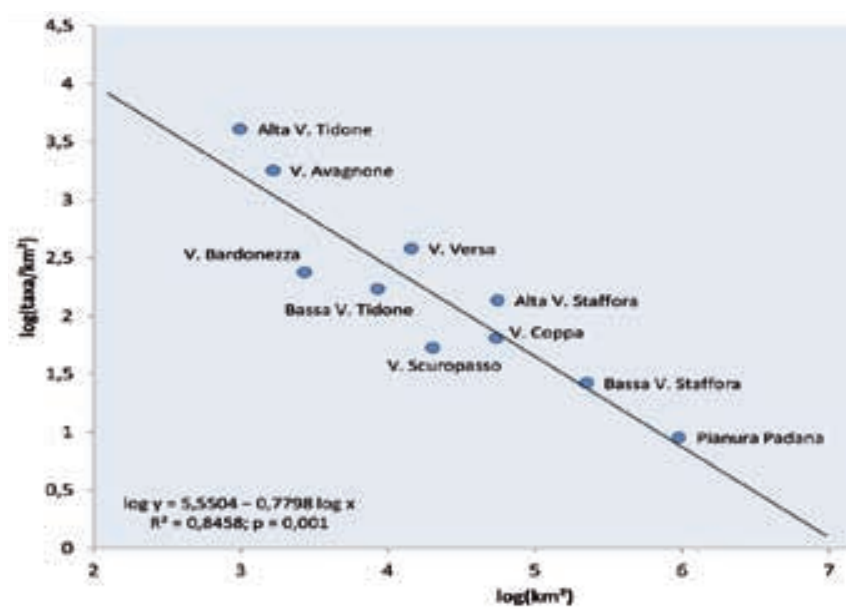

Fig. 13 - Flora dei settori oltrepadani. Regressione lineare, con relativa formula (secondo Cristofolini, 1998), tra il logaritmo delle superficie (in $\left.\mathrm{km}^{2}\right)$ e il logaritmo della densità floristica $\left(\operatorname{taxa} / \mathrm{km}^{2}\right)$ (grafico realizzato in collaborazione con S. Mossini). 
viticoltura intensiva; il vigneto domina anche i paesaggi delle adiacenti Valli Scuropasso e Bardonezza, dove la percentuale di taxa non più riconfermati appare però più bassa $(8,4 \%$ e $3,9 \%$ rispettivamente) presumibilmente a causa della minore disponibilità di dati floristici pregressi. La bassa precentuale della Pianura Padana $(14,2 \%)$ si deve invece all'ingresso delle esotiche, che ha compen- sato nel tempo la consistente riduzione del contingente autoctono (Tab. 3).

Tra le famiglie più rappresentate della flora oltrepadana dominano le Asteraceae (222 taxa), le Poaceae (171 taxa) e le Fabaceae (129 taxa); tra i generi, invece, il contributo più elevato in termini di specie e sottospecie è fornito da Carex (35), Trifolium (25) ed Euphorbia (22) (Tab. 4).

Tab. 3 - Prospetto numerico della flora dei dieci settori oltrepadani. $\mathrm{Km}^{2}=$ superficie (approssimata al $\mathrm{km}$ ); Densità floristica $=$ densità floristica $\left(\operatorname{taxa} / \mathrm{km}^{2}\right.$; cfr. Cristofolini, 1998) osservata (oss), attesa (att) e rapporto tra osservata e attesa (oss/att) per ogni settore (in grassetto i valori $\geq 1$ ); $+=$ taxa accertati dopo il 1980; $0=$ taxa non più ritrovati dopo il $1980 ; \% 0=$ percentuale dei taxa non più ritrovati di ogni settore; $\% 0 \mathrm{OP}=$ percentuale dei taxa non più ritrovati in ogni settore rispetto al totale dei taxa non più ritrovati in Oltrepò Pavese.

\begin{tabular}{|c|c|c|c|c|c|c|c|c|c|}
\hline \multirow{2}{*}{ Settore } & \multirow{2}{*}{$\mathbf{K m}^{2}$} & \multirow{2}{*}{ Taxa } & \multicolumn{3}{|c|}{ Densità floristica } & \multirow{2}{*}{+} & \multirow{2}{*}{$\mathbf{0}$} & \multirow{2}{*}{$\% 0$} & \multirow{2}{*}{$\% 00 P$} \\
\hline & & & oss & att & oss/att & & & & \\
\hline Pianura Padana & 395 & 1.024 & 2,592 & 2,431 & 1,066 & 878 & 146 & 14,3 & 42,6 \\
\hline Bassa Valle Staffora & 211 & 874 & 4,142 & 3,964 & 1,045 & 774 & 100 & 11,4 & 21,9 \\
\hline Valle Coppa & 114 & 696 & 6,105 & 6,406 & 0,953 & 591 & 105 & 15,1 & 14,8 \\
\hline Bassa Val Tidone & 51 & 475 & 9,314 & 11,995 & 0,776 & 450 & 25 & 5,3 & 2,7 \\
\hline Valle Scuropasso & 74 & 415 & 5,608 & 8,973 & 0,625 & 380 & 35 & 8,4 & 3,8 \\
\hline Valle Versa & 64 & 844 & 13,188 & 10,049 & 1,312 & 687 & 157 & 18,6 & 13,7 \\
\hline Valle Bardonezza & 31 & 333 & 10,742 & 17,685 & 0,607 & 320 & 13 & 3,9 & 0,0 \\
\hline Alta Valle Staffora & 115 & 971 & 8,443 & 6,363 & 1,327 & 830 & 141 & 14,5 & 27,9 \\
\hline Valle Avagnone & 25 & 647 & 25,880 & 20,915 & 1,237 & 579 & 68 & 10,5 & 8,2 \\
\hline Alta Val Tidone & 20 & 738 & 36,900 & 24,890 & 1,482 & 716 & 22 & 3,0 & 6,0 \\
\hline Oltrepò Pavese & 1.100 & 1.871 & 1,701 & 1,361 & 1,250 & 1.688 & 183 & 9,8 & - \\
\hline
\end{tabular}

Tab. 4 - Famiglie e generi maggiormente rappresentati (rispettivamente con più di 50 o 15 specie e sottospecie) nella flora dell'Oltrepò Pavese.

\begin{tabular}{|l|c|l|c|}
\hline Famiglie $>\mathbf{5 0}$ & Tot & Generi $\mathbf{1 5}$ & Tot \\
\hline Asteraceae & 222 & Carex & 35 \\
\hline Poaceae & 171 & Trifolium & 25 \\
\hline Fabaceae & 129 & Euphorbia & 22 \\
\hline Rosaceae & 84 & Vicia & 19 \\
\hline Brassicaceae & 76 & Rosa & 18 \\
\hline Lamiaceae & 72 & Veronica & 17 \\
\hline Apiaceae & 64 & Viola & 17 \\
\hline Caryophyllaceae & 63 & Festuca & 16 \\
\hline Cyperaceae & 62 & Galium & 16 \\
\hline Orchidaceae & 53 & Lathyrus & 16 \\
\hline
\end{tabular}




\section{Novità e conferme floristiche}

Vengono di seguito elencate le novità e le conferme floristiche che consentono di aggiornare la checklist della flora vascolare italiana (Conti et al., 2005, 2007) e la flora esotica lombarda (Banfi \& Galasso, 2010). A lato di ciascun nome viene specificato tra parentesi quadre se il taxon è un endemita italiano ("End"; cfr. Peruzzi et al., 2014, 2014 onwards, 2015) oppure un'entità alloctona a livello nazionale ("Neo" = neofita, "Arch" = archeofita, "Ama" = amaurogena) o regionale ("Loc.esot"). Per queste ultime due categorie viene riportato anche lo status d'invasività ("cas" = casuale, "nat" = naturalizzato, "inv" = invasivo); una freccia (" $\rightarrow$ ") sintetizza il cambiamento di status.

Entità nuove per l'Italia (4):

Bidens triplinervia Kunth [Neo cas]

Cucurbita melopepo L. subsp. melopepo [Neo cas]

Morus kagayamae Koidz. [Neo cas]

$\times$ Triticosecale Wittm. ex A.Camus [Arch cas]

Vitis berlandieri Planch. $\times$ V. vinifera $\mathrm{L}$. [Neo nat] (Fig. 14)

Entità nuove per la Lombardia (26):

Allium polyanthum Schult. \& Schult.f. [Loc.esot nat]

Aquilegia cultivar [Neo cas]

Aubrieta columnae Guss. s.1. [Loc.esot nat]

Cota tinctoria (L.) J.Gay subsp. australis (R.Fern.) Ober-

pr. \& Greuter

Crepis leontodontoides All.

Dianthus longicaulis Ten.

Festuca maritima L.

Hieracium chlorelloides Zahn

Hieracium grovesianum Arv.-Touv. ex Belli s.l. [End]

Hieracium pellitum Fr.

Hyssopus officinalis L. subsp. officinalis

Kickxiaelatine(L.)Dumort.subsp.crinita(Mabille)Greuter

Lilium candidum L. [Ama cas]
Loncomelos pyrenaicum (L.) L.D.Hrouda ex Holub subsp. sphaerocarpum (A.Kern.) Holub

Lycopsis orientalis L. [Neo nat] (Fig. 15B)

Pilosella leptophyton (Nägeli \& Peter) S.Bräut. \& Greuter

Pinus halepensis Mill. [Loc.esot cas]

Pteris vittata $\mathrm{L}$. [Neo cas]

Pyracantha crenatoserrata (Hance) Rehder [Neo cas]

Scabiosa mollissima Viv.

Spinacia oleracea L. [Arch cas]

Stipa etrusca Moraldo [End]

Telekia speciosa (Schreb.) Baumg. [Neo nat]

Tetragonia tetragonoides (Pall.) Kuntze [Neo cas]

Triticum $\times$ requienii Ces., Pass. \& Gibelli [Arch cas]

Viola calcarata L. subsp. cavillieri (W.Becker) Negodi

Entità nuove per la Lombardia, non ritrovate di recente (4):

Colymbada rupestris (L.) Holub [Loc.esot]

Digitalis laevigata Waldst. \& Kit. subsp. laevigata [Loc. esot]

Galatella sedifolia (L.) Greuter subsp. sedifolia

Kali macrophyllum (R.Br.) Galasso \& Bartolucci

Entità confermate per la Lombardia (12):

Adenostyles australis (Ten.) Iamonico \& Pignatti [End]

Brachypodium genuense (DC.) Roem. \& Schult. [End]

Bromopsis erecta (Huds.) Fourr. subsp. stenophylla (Link)

H.Scholz \& Valdés

Carduus defloratus L. subsp. carlinifolis (Lam.) Ces.

Elymus acutus (DC.) M.-A.Thiébaud

Hainardia cylindrica (Willd.) Greuter

Lepidium latifolium L. [Loc.esot cas]

Pinus pinea L. [Loc.esot cas]

Tephroseris italica Holub [End]

Tragopogon eriospermus Ten.

Vicia bithynica $(\mathrm{L}$.) L.

Vicia narbonensis L. subsp. serratifolia (Jacq.) Ces.
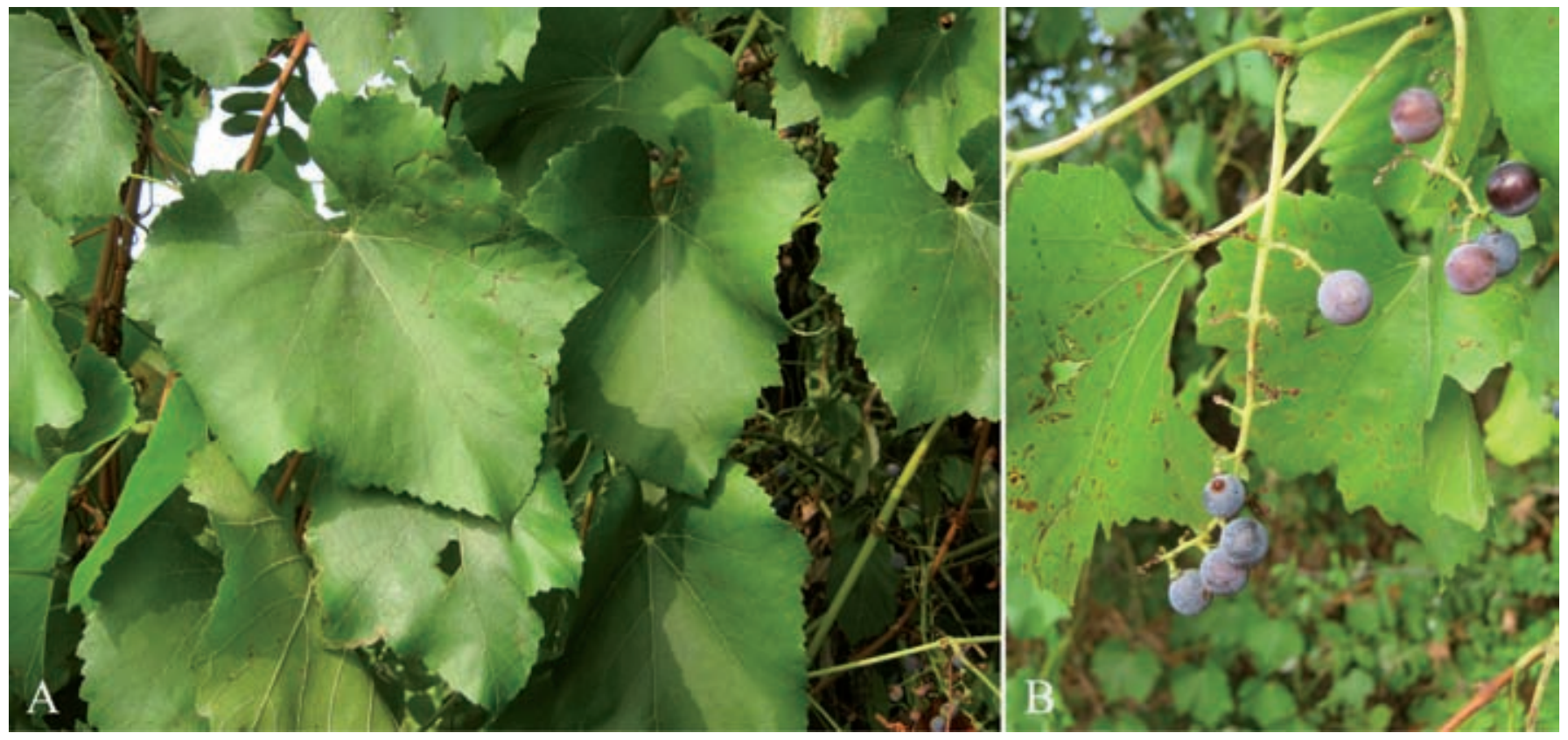

Fig. 14 - Vitis berlandieri Planch. $\times$ V. vinifera L. '41 B' naturalizzata a Montù Beccaria: A) lamine fogliari; B) infruttescenza (Foto: Nicola M.G. Ardenghi). 

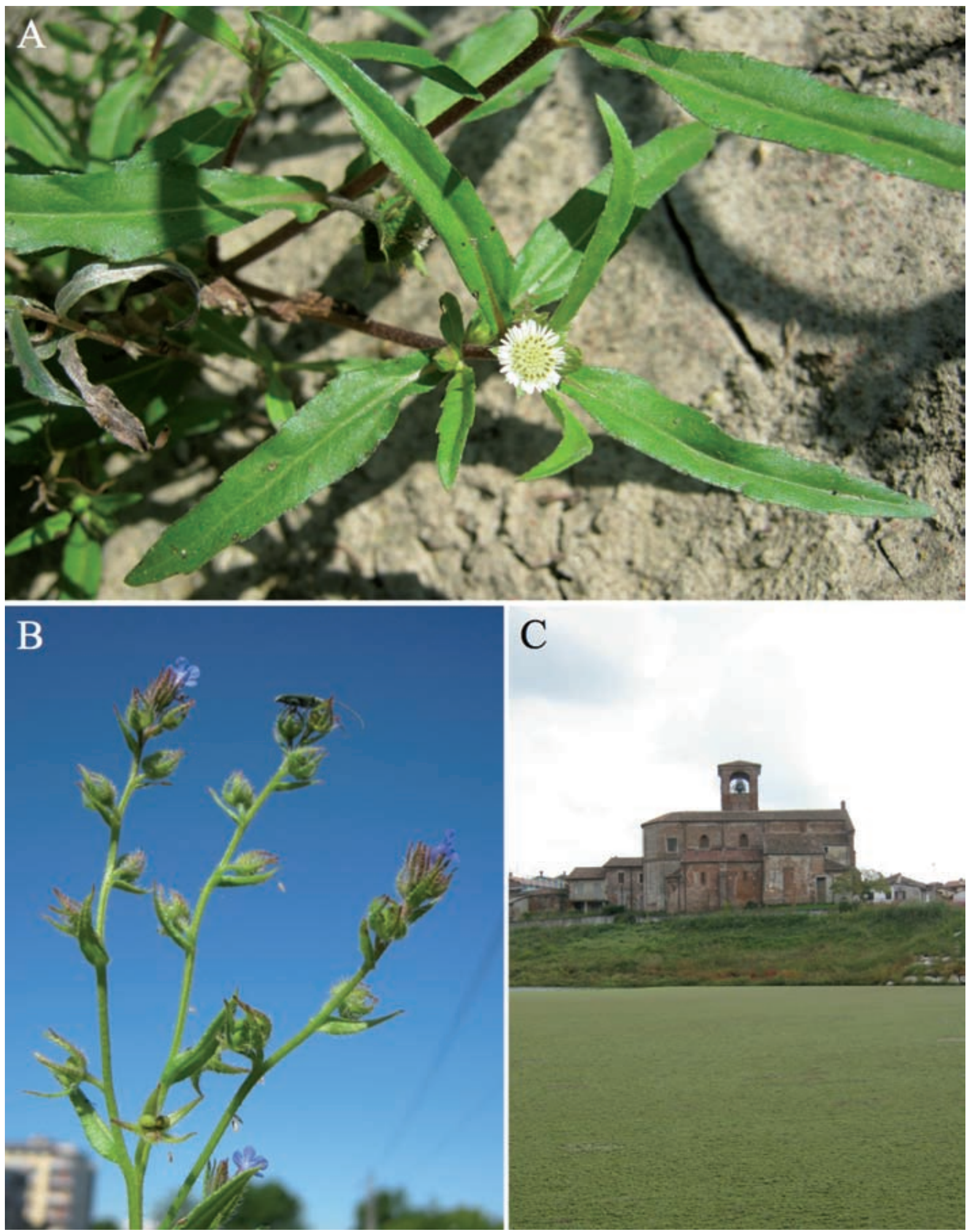

$\mathrm{C}$

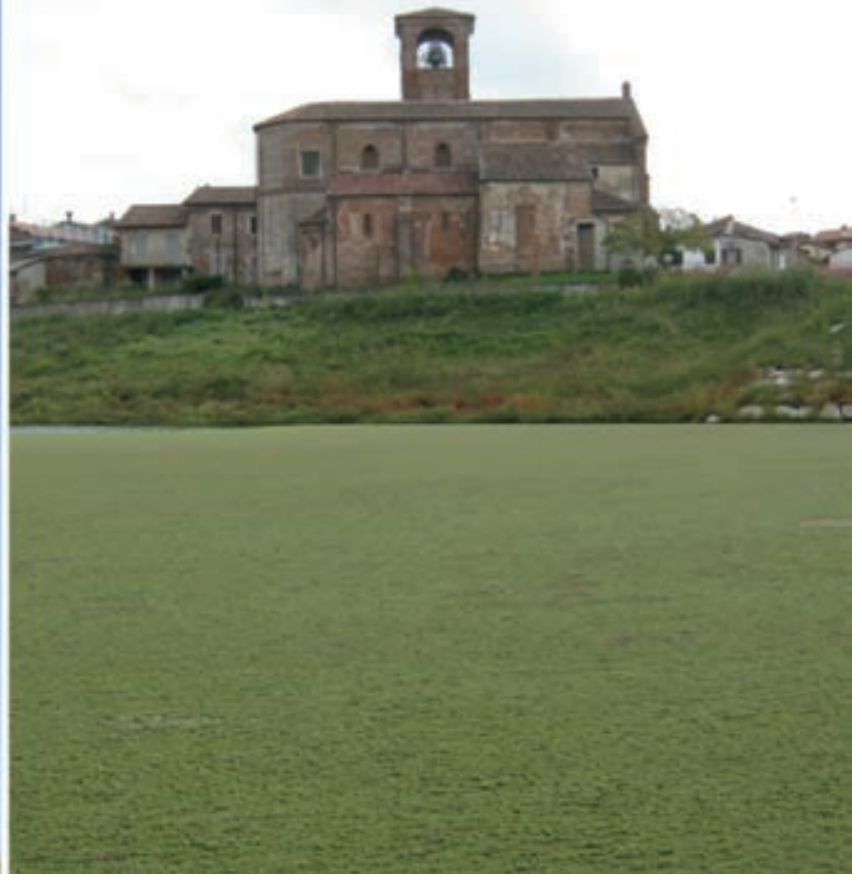

Fig. 15 - Nuove neofite e cambiamenti di status: A) Eclipta prostrata (L.) L. lungo la sponda nord del Po a Bastida Pancarana; B) Lycopsis orientalis L. lungo il torrente Staffora a Voghera; C) Azolla filiculoides Lam. letteralmente tappezzante la lanca dello Scolo Cardanile ad Arena Po (Foto: Nicola M.G. Ardenghi). 
Entità confermate per la Lombardia, non ritrovate di recente (4):

Centaurea aplolepa Moretti s.l. [End]

Centaurea aspera L. subsp. aspera

Hieracium racemosum Waldst. \& Kit. ex Willd. subsp. virgaurea (Coss.) Zahn

Malva nicaeensis L. [Loc.esot]

Entità alloctone nuove per la provincia di Pavia (16):

Acanthus mollis L. subsp. mollis [Ama cas]

Amaranthus caudatus L. [Neo cas]

Amaranthus emarginatus Salzm. ex Uline \& W.L.Bray

[Neo nat]

Berberis thunbergii DC. [Neo cas]

Ceratostigma plumbaginoides Bunge [Neo cas]

Coriandrum sativum L. [Arch cas]

Ginkgo biloba L. [Neo cas]

Guizotia abyssinica (L.f.) Cass. [Neo cas]

Helianthus $\times$ laetiflorus Pers. (pro sp.) [Neo nat]

Iris squalens L. [Neo cas]

Oxalis latifolia Kunth [Neo cas]

Paspalum dilatatum Poir. [Neo cas]

Ulmus laevis Pall. [Neo nat]

Triticum turgidum L. subsp. durum (Desf.) Husn. [Arch cas]

Vitis $\times$ bacoi Ardenghi, Galasso \& Banfi [Neo nat]

Entità alloctone confermate per la provincia di Pavia (6):

Cucurbita pepo L. subsp. pepo [Neo cas]

Digitaria ciliaris (Retz.) Koeler [Neo nat]

Jasminum officinale L. [Arch cas]

Mespilus germanica L. [Ama nat]

Nepeta cataria L. [Ama cas]

Prunus dulcis (Mill.) D.A.Webb. [Arch cas]

Cambiamento di status d'invasività per la Lombardia (7):

Alcea rosea $\mathrm{L}$. [Neo cas $\rightarrow$ nat]

Amaranthus emarginatus Salzm. ex Uline \& W.L.Bray

[Neo cas $\rightarrow$ nat]

Anthriscus cerefolium (L.) Hoffm. [Arch cas $\rightarrow$ nat]

Azolla filiculoides Lam. [Neo nat $\rightarrow$ inv] (Fig. 15C)

Eclipta prostrata (L.) L. [Neo cas $\rightarrow$ nat] (Fig. 15A)

Trifolium alexandrinum $\mathrm{L}$. [Ama cas $\rightarrow$ nat]

Vitis $\times$ instabilis Ardenghi, Galasso, Banfi \& Lastrucci

[Neo nat $\rightarrow$ inv $]$

Cambiamento di status d'invasività per la provincia di Pavia (3):

Antirrhinum majus L. subsp. majus [Arch cas $\rightarrow$ nat]

Rumex cristatus DC. [Ama nat $\rightarrow$ inv]

Syringa vulgaris L. [Neo nat $\rightarrow$ cas]

\section{Origine geografica e tipi biologici}

La collocazione dell'Oltrepò Pavese nella regione biogeografica Eurosiberiana è fedelmente rispecchiata dall'origine geografica dei taxa che compongono la sua flora (Fig. 16). Le entità di origine eurasiatica $(13,7 \%)$, europea $(9,7 \%)$, circumboreale $(6 \%)$ ed eurosiberiana $(5,5 \%)$, che insieme rappresentano il $34,9 \%$ dei taxa, denotano infatti una rilevante continentalità della flora oltrepadana, che sovrasta il carattere mediterraneo conferito dai taxa di origine euri- $(13,6 \%)$ e steno-mediterranea $(4,8 \%)$, alcuni dei quali estranei al contingente autoctono. La componente orofitica, rappresentata dalle orofite sud-europee $(7,7 \%)$ e dalle mediterraneo-montane $(2 \%)$, è piuttosto contenuta, in linea con la limitata estensione dei rilievi montani. L'antropizzazione della pianura e della bassa collina è invece rivelata dalla percentuale dei taxa di origine americana $(6,5 \%)$ e asiatica $(3,9 \%)$ che costituiscono da soli il $10,4 \%$ della flora.

Lo spettro biologico (Fig. 17) è contraddistinto da una predominanza di emicriptofite $(38,7 \%)$, che conferma il carattere continentale della flora già evidenziato dall'origine geografica. Notevole è anche la presenza di terofite $(25,1 \%)$, legate agli ambienti xerici di collina e ai contesti ruderali planiziali. L'esigua percentuale di idrofite $(1,9 \%)$ è un'ulteriore riprova della scarsità di aree umide in Oltrepò Pavese.

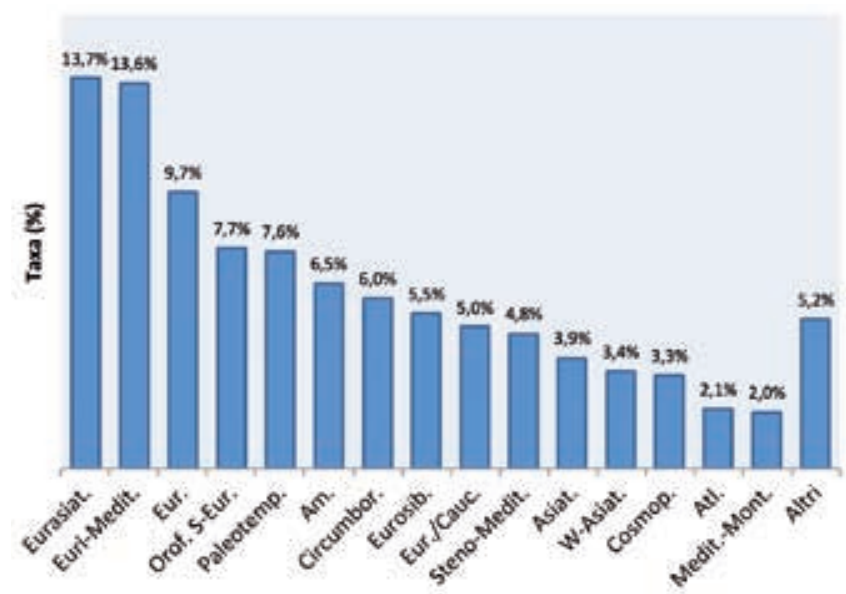

Fig. 16 - Origine geografica dei taxa costituenti la flora dell'Oltrepò Pavese.

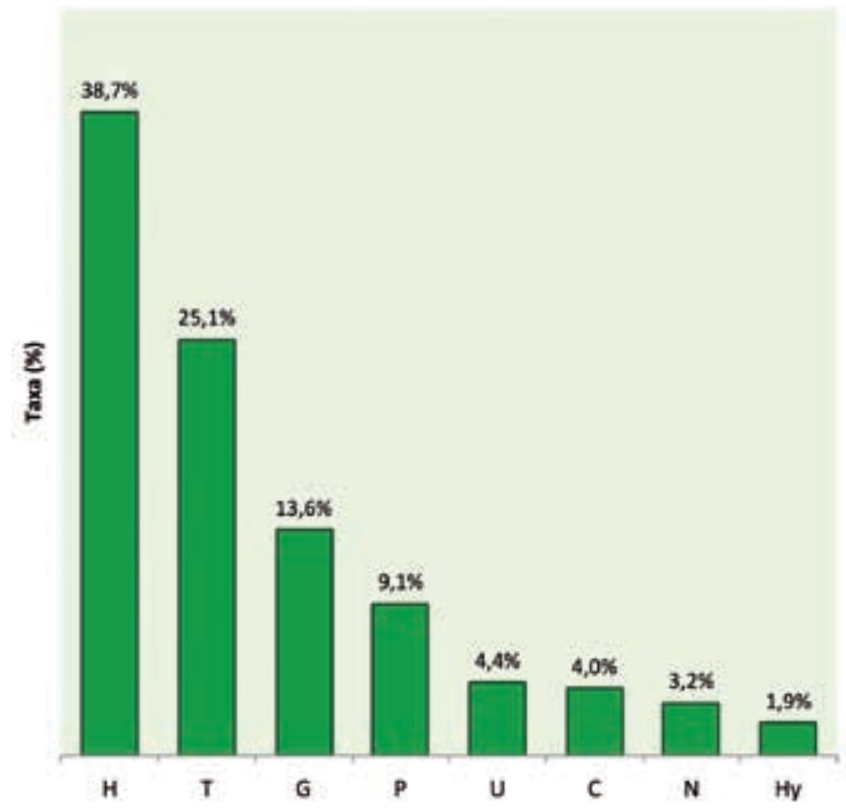

Fig. 17 - Tipi biologici della flora dell'Oltrepò Pavese. 


\section{Endemiti italiani}

In Oltrepò Pavese sono stati censiti 16 endemiti italiani: Adenostyles australis (Ten.) Iamonico \& Pignatti Anemonoides trifolia (L.) Holub subsp. brevidentata

(Ubaldi \& Puppi) Galasso, Banfi \& Soldano

Astragalus sirinicus Ten. subsp. sirinicus

Brachypodium genuense (DC.) Roem. \& Schult.

Centaurea aplolepa Moretti s.l.

Cerastium tomentosum L. [Loc.esot nat]

Cherleria laricifolia (L.) Iamonico subsp. ophiolitica (Pi-

gnatti) Iamonico (Fig. 11C)

Crocus biflorus Mill.

Hieracium grovesianum Arv.-Touv. ex Belli s.l.

Melampyrum italicum (Beauverd) Soó

Odontarrhena argentea (All.) Ledeb. (Fig. 11B)

Phyteuma ovatum Honck. subsp. pseudospicatum Pignatti

Robertia taraxacoides (Loisel.) DC.

Sesleria pichiana (Savi) Savi

Stipa etrusca Moraldo

Tephroseris italica Holub

Uno di essi (Centaurea aplolepa s.1.) non è stato più ritrovato in tempi recenti e un altro (Cerastium tomentosum) è stato introdotto a scopo ornamentale; a differenza di Peruzzi et al. (2014, 2014 onwards, 2015), Tragopogon eriospermus, accertato per l'area di studio, non viene considerato un taxon endemico italiano (cfr. Tison et al., 2004; Euro+Med, 2006 onwards). Gli endemiti oltrepadani costituiscono da soli la quasi totalità del contingente endemico italiano presente in provincia di Pavia, rappresentato a nord del Po esclusivamente da Isoëtes malinverniana Ces. \& De Not. (Ardenghi, 2015b).

Il numero degli endemiti italiani presenti in Oltrepò Pavese è molto basso se rapportato a quello regionale, che è pari a 82 [la cifra, rispetto a Galasso (2015a, 2015b), comprende anche le 6 novità/conferme regionali qui segnalate oltre a Cerastium tomentosum e a Crocus biflorus (cfr. Harpke et al., 2016)]. Nessuno di essi è esclusivo dell'area oltrepadana, tuttavia, 13 non si trovano in nessun'altra provincia lombarda (Cerastium tomentosum, Crocus biflorus e $\mathrm{Me}$ lampyrum italicum sono invece noti in altre province; cfr., ad es., Soó, 1926, Banfi \& Galasso, 1998, Giordana, 2010a, e Martini et al., 2012), trattandosi principalmente di taxa a distribuzione appenninica o sudovest-alpica-appenninica.

\section{Taxa protetti}

Nell'area di studio risultano presenti 16 taxa elencati nella Lista Rossa della Flora Italiana (Rossi et al., 2013): Myricaria germanica, Sagittaria sagittifolia Willd., Typha minima, EN (minacciata); Salvinia natans (L.) All., VU (vulnerabile); Epipactis palustris, Fritillaria montana, Gentiana lutea L. subsp. lutea, Trapa natans L., Typha shuttleworthii W.D.J.Koch \& Sond., NT (quasi a rischio); Arnica montana subsp. montana, Asplenium adulterinum subsp. adulterinum, Galanthus nivalis L., Himantoglossum adriaticum, Lycopodium clavatum, Ruscus aculeatus L., LC (a minor rischio); Lycopodium alpinum L., DD (dati insufficienti). Tra questi figurano 13 policy species, due delle quali (Asplenium adulterinum subsp. adulterinum e $\mathrm{Hi}$ mantoglossum adriaticum) incluse nell'allegato II della dir. n. 92/43/CEE (Direttiva "Habitat"); altre 6 policy species sono invece elencate nell'allegato $\mathrm{V}$ della stessa direttiva.
5 entità non sono state più ritrovate dopo il $1980(L y$ copodium alpinum, L. clavatum, Myricaria germanica, Trapa natans, Typha minima), tra cui figurano 2 dei 3 taxa appartenenti alla categoria "EN". Delle 11 entità accertate in tempi recenti, solo Sagittaria sagittifolia e Arnica montana subsp. montana sono state rinvenute all'interno di due dei tre siti Rete Natura 2000 presenti in Oltrepò Pavese, rispettivamente nella ZPS IT2080701 "Po da Albaredo Arnaboldi ad Arena Po" e nel SIC IT2080021 "Monte Alpe"; nessun taxon è stato invece rilevato nel terzo sito, il SIC IT2080020 "Garzaia della Roggia Torbida" (Perracino, 2010; Casale et al., 2011). Arnica montana subsp. montana e Gentiana lutea subsp. lutea sono presenti nel territorio della Riserva naturale "Le Torraie-Monte Lesima", mentre ulteriori entità potrebbero rientrare nell'area denominata "Sassi Neri-Pietra Corva", candidata quale sito di importanza comunitaria (d.g.p. Provincia di Pavia del 27 maggio 2014, n. 188/32965). Salvinia natans è stata osservata nel Parco Lombardo della Valle del Ticino e precisamente a Mezzanino, l'unico comune oltrepadano (assieme alle porzioni di Linarolo e Travacò Siccomario oltrepassanti il Po) appartenente al Parco.

Un numero più consistente di taxa risulta incluso negli elenchi C1 (163) e C2 (67) della 1.r. Lombardia n. 10/2008.

\section{Taxa alloctoni}

La flora alloctona dell'Oltrepò Pavese annovera un totale di 256 neofite, 65 archeofite, 22 amaurogene e 86 esotiche locali. Le esotiche a livello nazionale (archeofite e neofite) costituiscono il $17,2 \%$ della flora oltrepadana (Tab. 5), valore prossimo alla percentuale della Lombardia (17,3\%; cfr. Galasso, 2015a). Tra queste figurano 19 entità inserite nella Lista Nera della flora lombarda (1.r. Lombardia n. 10/2008).

Confrontando i dati attuali con quelli dell'ultima flora provinciale (Rota, 1847), le esotiche nazionali sono passate da 65 a 321, con un incremento pari al 393,8\%. Ai tempi di Rota le archeofite predominavano sulle neofite ma il loro aumento è stato più contenuto rispetto a quello delle neofite (Tab. 6).

Analizzando la flora dei singoli settori, le percentuali più elevate di esotiche (superiori a quella dell'intero Oltrepò Pavese) si riscontrano in Pianura Padana $(24,9 \%)$, e in Valle Versa (21\%); appaiono invece del tutto irrisorie quelle relative ai settori montani, ovvero Valle Avagnone (2,2\%), alta Valle Staffora e alta Val Tidone (entrambi 4,7\%). In Pianura Padana e in Valle Versa si concentra più della metà della flora esotica oltrepadana $(79,4 \%$ e $55,1 \%$ rispettivamente) ed è in questi due settori e in Valle Bardonezza dove si rileva la maggior parte delle neofite e delle archeofite invasive censite nell'intera area di studio $(100 \%, 86,7 \%$ e $63,3 \%$ rispettivamente) (Tab. 5). Come prevedibile, l'invasione di specie vegetali aliene colpisce in modo più vistoso la Pianura $\mathrm{Pa}-$ dana, che ospita le aree urbanizzate di maggiori dimensioni, i principali assi viari e il fiume Po, vettore naturale per la diffusione delle esotiche su scala regionale; risultano particolarmente interessati da questo fenomeno anche i settori collinari orientali, in particolare le Valli Versa e Bardonezza, dove la bassa naturalità indotta dalla viticoltura intensiva non è in grado di ostacolare l'insediamento e l'espansione delle aliene provenienti dalla limitrofa pianura. 
Tab. 5 - Prospetto numerico della flora alloctona dell'Oltrepò Pavese. Neo = neofite; Arch = archeofite; Ama $=$ amaurogene; Loc.Esot $=$ esotiche a livello locale; $\%$ Esot $=$ percentuale dei taxa esotici (neofite + archeofite) di ogni settore rispetto alla sua flora; \%Fl.Esot. = percentuale dei taxa esotici (neofite + archeofite) in ogni settore rispetto al totale delle esotiche oltrepadane; \%Esot.Inv = percentuale dei taxa esotici invasivi (neofite + archeofite) in ogni settore rispetto al totale dei taxa invasivi oltrepadani; Aut = taxa autoctoni a livello locale; \%Aut = percentuale dei taxa autoctoni a livello locale di ogni settore rispetto alla sua flora.

\begin{tabular}{|c|c|c|c|c|c|c|c|c|c|c|}
\hline \multirow[b]{2}{*}{ Settore } & \multirow[b]{2}{*}{ Neo } & \multirow[b]{2}{*}{ Arch } & \multirow[b]{2}{*}{ Ama } & \multirow{2}{*}{$\begin{array}{l}\text { Loc. } \\
\text { Esot }\end{array}$} & \multicolumn{4}{|c|}{ Esot (Neo+Arch) } & \multicolumn{2}{|c|}{ Aut } \\
\hline & & & & & Tot & \% Esot & $\begin{array}{c}\% \text { Fl. } \\
\text { Esot }\end{array}$ & $\begin{array}{l}\text { \% Esot. } \\
\text { Inv }\end{array}$ & Tot & $\%$ Aut \\
\hline Pianura Padana & 206 & 49 & 19 & 54 & 255 & 24,9 & 79,4 & 100,0 & 696 & 68,0 \\
\hline Bassa Valle Staffora & 66 & 26 & 12 & 23 & 92 & 10,5 & 28,7 & 56,7 & 747 & 85,5 \\
\hline Valle Coppa & 52 & 23 & 12 & 22 & 75 & 10,8 & 23,4 & 50,0 & 587 & 84,3 \\
\hline Bassa Val Tidone & 28 & 12 & 3 & 10 & 40 & 8,4 & 12,5 & 31,7 & 422 & 88,8 \\
\hline Valle Scuropasso & 47 & 15 & 9 & 13 & 62 & 14,9 & 19,3 & 45,0 & 331 & 79,8 \\
\hline Valle Versa & 143 & 34 & 13 & 36 & 177 & 21,0 & 55,1 & 86,7 & 618 & 73,2 \\
\hline Valle Bardonezza & 47 & 9 & 8 & 9 & 56 & 16,8 & 17,4 & 63,3 & 260 & 78,1 \\
\hline Alta Valle Staffora & 32 & 14 & 8 & 24 & 46 & 4,7 & 14,3 & 25,0 & 893 & 92,0 \\
\hline Valle Avagnone & 6 & 8 & 2 & 8 & 14 & 2,2 & 4,4 & 6,7 & 623 & 96,3 \\
\hline Alta Val Tidone & 21 & 14 & 4 & 13 & 35 & 4,7 & 10,9 & 11,7 & 686 & 93,0 \\
\hline Oltrepò Pavese & 256 & 65 & 22 & 86 & 321 & 17,2 & - & - & 1.442 & 77,1 \\
\hline
\end{tabular}

Tab. 6 - Confronto tra il numero di taxa esotici (neofite + archeofite) riportati da Rota (1847) per l'Oltrepò Pavese e quello attuale (taxa accertati e non più ritrovati dopo il 1980). Tot 1847 = taxa esotici riportati da Rota (1847).

\begin{tabular}{|l|c|c|c|c|}
\hline & Tot 1847 & Tot attuale & Incremento & Incremento \% \\
\hline Neofite & 26 & 256 & 230 & 884,6 \\
\hline Archeofite & 39 & 65 & 26 & 66,7 \\
\hline Amaurogene & 12 & 22 & 10 & 83,3 \\
\hline Loc. esotiche & 23 & 86 & 63 & 273,9 \\
\hline Esotiche (Arch+Neo) & 65 & 321 & 256 & 393,8 \\
\hline
\end{tabular}

\section{Ringraziamenti}

Gli autori ringraziano sentitamente Gabriele Galasso ed Enrico Banfi (Museo di Storia Naturale di Milano), per i preziosi consigli nomenclaturali forniti durante l'intero periodo di redazione dell'opera. Un grazie speciale è rivolto ai seguenti specialisti che hanno revisionato i reperti di alcuni gruppi critici e fornito suggerimenti in merito alla loro trattazione: Fabrizio Bartolucci (Università di Camerino - Centro Ricerche Floristiche dell'Appennino, Parco Nazionale del Gran Sasso e Monti della Laga, Barisciano), Thymus; Valentino Casolo (Università di Udine), Achillea millefolium (group); Günter Gottschlich (Tübingen, Germania), Hieracium e Pilosella; Lorenzo Peruzzi (Università di Pisa), Crocus; Edward E. Schilling (University of Tennessee, Stati Uniti), Helianthus. Esprimiamo la nostra gratitudine anche a: Sara Mossini (Terdobbiate), per il supporto nell'elaborazione statistica; Claudio Ballerini (Trivolzio), co-autore delle mappe; Giulia Ardenghi (Stradella), per l'elaborazione digitale delle figure; Graziano Ardenghi
(Eni S.p.A.), per la rilettura critica del paragrafo introduttivo "Geologia"; Adriano Soldano (Vercelli), per aver permesso la consultazione degli appunti originali di Vincenzo de Cesati; Chiara Nepi, Egildo Luccioli, Lorenzo Cecchi (FI) e il personale dell'Archivio di Stato di Piacenza per l'assistenza nella consultazione delle rispettive collezioni erbariologiche; Maurizio Preti (Pavia), per l'aiuto fornito nelle primissime fasi della ricerca in PAV; Francesca Cattaneo, Giuseppe De Paolis, Anna Letizia Magrassi, Arturo Peloso e Rosanna Zavatarelli (Biblioteca della Scienza e della Tecnica, Università degli Studi di Pavia), per l'aiuto nella ricerca bibliografica; il forum online Acta Plantarum (<http://www.actaplantarum.org/>), fonte inesauribile di spunti e informazioni; Anna Alessandrello (Museo di Storia Naturale di Milano), per l'assistenza editoriale, e Michela Mura (Museo di Storia Naturale di Milano), per l'ammirevole pazienza e professionalità nell'impaginazione finale dell'opera. La flora sarebbe risultata più scarna e meno accattivante senza il supporto logistico e i dati personali mes- 
si a disposizione dalle seguenti persone, a cui va la nostra più sincera riconoscenza: Thomas Abeli (Università degli Studi di Pavia), Alvaro Albanesi (Montù Beccaria), Rosa Armenio (Stradella), Silvia Assini (Università degli Studi di Pavia), Matteo Barcella (Cologno Monzese), Giovanni Barcheri (Siziano), Enrico Bizzagni (Casteggio), Silvia Bodino (Università degli Studi di Pavia), Fabrizio Bonali (Sesto ed Uniti), Luciano Bongiorni (Gazzola), il sig. Bossi (San Cipriano Po), Guido Brusa (Varese), Paolo Cauzzi (Università degli Studi di Pavia), Marco Consiglio (Pavia), Carlo Cova (San Damiano al Colle), Elena Filipponi (Arena Po), Luca Gariboldi (Rho), Carolina Girometta (Università degli Studi di Pavia), Filippo Guzzon (Università degli Studi di Pavia), Giovanni Manstretta (Arena Po), Sara Martimucci (Rivanazzano Terme), Simone Orsenigo (Università degli Studi di Milano), Gilberto Parolo (Campospinoso), Simone Pedrini (Curtin University, Perth), Nicola Pilon (Milano), Enrico Romani (Museo civico di Storia naturale di Piacenza), Graziano Rossi (Università degli Studi di Pavia), Matilde Nives Rossi (Carbonara al Ticino), Emanuele Vegini (Bornasco), Filip Verloove (Botanic Garden of Meise, Belgio). Un rinnovato ringraziamento è infine riservato a Gabriele Galasso e Fabrizio Bartolucci, per il valoroso impegno nella revisione del manoscritto.

\section{BIBLIOGRAFIA}

Abeli T., Parolo G. \& Dell'Orto V., 2012 - Orchidee spontanee dell'Appennino Pavese. Guida al riconoscimento, carte di distribuzione e itinerari di scoperta. Nuova Tipografia Popolare, Pavia.

Allioni C., 1785 - Flora pedemontana. I.M. Briolus, Torino, 1-3.

Amici del Parco Le Folaghe, 2009 - Parco Le Folaghe. <http://www. parcolefolaghe.it> (ultima consultazione 13 dicembre 2015).

Andreis C. \& Sartori F., 2011 - Vegetazione forestale della Lombardia. Inquadramento fitosociologico. Archivio Geobotanico, 12-13 (2006-2007): 1-215.

Ardenghi N.M.G., 2012 - Notulae 51-94. In: Notulae ad plantas advenas longobardiae spectantes: 2 (29-140). Galasso G. \& Banfi E. (eds.). Pagine Botaniche, 35 (2011): 58-78.

Ardenghi N.M.G., 2013a - Cyperus schweinitzii (Cyperaceae): a new naturalized alien in Italy. Webbia, 68 (1): 73-76. doi: 10.1080/00837792.2013.779815

Ardenghi N.M.G., 2013b - Herbarium Universitatis Ticinensis (PAV): segnalazioni interessanti per la flora dell'Italia nord-occidentale. Informatore Botanico Italiano, 45 (1): 45-52.

Ardenghi N.M.G., 2013c - Filippo Morandini (1826-1903), botanico e patriota risorgimentale: inedite annotazioni per il Pavese, il Lodigiano e il Colle San Colombano. Pianura, scienze e storia dell'ambiente padano, 30: 3-50.

Ardenghi N.M.G., 2014 - Le raccolte di Luigi Ceroni (1883-1951) all'ex scalo merci ferroviario di Milano Porta Sempione (Lombardia, Italia). Pagine Botaniche, 37 (2013): 3-23.

Ardenghi N.M.G., 2015a - Verso la Flora della Provincia di Pavia: il risveglio dopo un inverno lungo due secoli. In: Approfondimenti floristici e sistematici sulla flora d'Italia. Dedicato a Edda Lattanzi in occasione dei suoi 85 anni. Comunicazioni. Orto botanico di Roma, La Sapienza Università di Roma, 20-21 novembre 2015. Peruzzi L. \& Domina G. (eds.). Società Botanica Italiana, Gruppo per la Floristica, Sistematica ed Evoluzione, Firenze: 37-38.

Ardenghi N.M.G., 2015b - La flora della provincia di Pavia: traguardi, prospettive e stato attuale delle conoscenze. In: Biodiversità nell'anno di Expo: la straordinaria flora lombarda. Atti della giornata di approfondimento delle conoscenze floristiche. Milano, 23 maggio 2015. Galasso G. \& Mangili F. (eds.). Natura, 105 (2): 71-76.

Ardenghi N.M.G. \& Mossini S., 2013 - Note su alcune esotiche a livello regionale in Lombardia (Italia). Pagine Botaniche, 36 (2012): 12-17.
Ardenghi N.M.G. \& Parolo G., 2012 - Spontanee tra i filari. Oltre, Bimestrale di cultura, ambiente e turismo, 135: 77-79.

Ardenghi N.M.G. \& Polani F., 2010a - Notulae 1728-1730. In: Notulae alla checklist della Flora vascolare Italiana: 10 (1682-1750). Nepi C., Peccenini S. \& Peruzzi L. (eds.). Informatore Botanico Italiano, 42 (2): 525-526.

Ardenghi N.M.G. \& Polani F., 2010b - Notula 49. In: Notulae alla checklist della Flora vascolare Italiana: 10 (1682-1750). Notulae alla flora esotica d'Italia: 3 (38-53). Nepi C., Peccenini S. \& Peruzzi L. (eds.). Informatore Botanico Italiano, 42 (2): 534.

Ardenghi N.M.G., Galasso G., Banfi E., Zoccola A., Foggi B. \& Lastrucci L., 2014 - A taxonomic survey of the genus Vitis (Vitaceae) in Italy, with special reference to Elba Island (Tuscan Archipelago). Phytotaxa, 166 (3): 163-198. doi: 10.11646/ phytotaxa.166.3.1

Ardenghi N.M.G., Banfi E. \& Galasso G., 2015 - A taxonomic survey of the genus Vitis L. (Vitaceae) in Italy, part II: the 'EuroAmerican' hybrids. Phytotaxa, 224 (3): 232-246. doi: 10.11646/ phytotaxa.224.3.2

Arrigoni P.V., 1983 - Aspetti corologici della flora sarda. Lavori della Società Italiana di Biogeografia, n. s., 8: 83-109.

Associazione Zucca Berrettina, 2014 - Quella strana berretta di Calcababbio. Storia del recupero della varietà Zucca Berrettina di Lungavilla (Pv). Italgrafica Srl, Novara.

Backlund A. \& Pyck N., 1998 - Diervillaceae and Linnaeaceae, two new family of caprifoliods. Taxon, 47 (3): 657-661.

Banfi E. \& Galasso G., 1998 - La flora spontanea della città di Milano alle soglie del terzo millennio e i suoi cambiamenti a partire dal 1700. Memorie della Società italiana di Scienze naturali e del Museo civico di Storia Naturale di Milano, 28 (1): 267-388.

Banfi E. \& Galasso G. (eds.), 2010 - La flora esotica lombarda. Museo di Storia Naturale di Milano, Milano.

Banti L., Gianatti G. \& Piazza G., 2015 - Flora del Lario da Giuseppe Comolli ai giorni nostri. New Press Edizioni, Cermenate.

Barcella M., Assini S. \& Ardenghi N.M.G., 2014 - A phytosociological survey of semi-dry mountain grasslands in the Northern Apennines (Italy). Atti del $48^{\circ}$ Congresso Scienza della Vegetazione e monitoraggio della Biodiversità, 17-19 settembre 2014, Roma.

Bergamaschi G., 1823a - Gita Botanica agli Appennini Boglelio, e Lesime. Giornale di Fisica, Chimica, Storia naturale, Medicina, ed Arti, s. 2, 6 (1): 69-75.

Bergamaschi G., 1823b - Gita Botanica agli Appennini Boglelio, e Lesime. Giornale di Fisica, Chimica, Storia naturale, Medicina, ed Arti, s. 2, 6 (2): 92-97.

Bergamaschi G., 1824a - Lettera seconda del Dottor Giuseppe Bergamaschi al sig. Prof. Giuseppe Moretti sopra varie piante degli Appennini, colli Oltrepadani, e della campagna pavese, da aggiungersi alla Flora Ticinese. Giornale di Fisica, Chimica, Storia naturale, Medicina, ed Arti, s. 2, 7 (3): 211-222.

Bergamaschi G., 1824b - Fine della lettera seconda del Dott. Giuseppe Bergamaschi al sig. Prof. D. Giuseppe Moretti sopra varie piante degli Appennini, colli Oltrepadani, e della campagna pavese, da aggiungersi alla Flora Ticinese. Giornale di Fisica, Chimica, Storia naturale, Medicina, ed Arti, s. 2, 7 (4): 266-281.

Bertolani-Marchetti D., 1954 - Il popolamento vegetale nelle stazioni salse della Valle Padana. Webbia, 9 (2): 511-621. doi: 10.1080/00837792.1954.10669622

Bertolotti S., 1954 - Le realizzazioni italiane nel campo della televisione. Elettronica, 3 (maggio-giugno 1954): 104-115.

Bianchi V. \& Pozzi G.C., 1959 - Una bega tra Nocca e Moretti. Atti dell'Istituto Botanico e del Laboratorio Crittogamico dell'Università di Pavia, s. 5, 16: 151-153.

Bianchi V., Bruno E. \& Giacomini V., 1959 - Giuseppe Moretti (Biobibliografia). Atti dell'Istituto Botanico e del Laboratorio Crittogamico dell'Università di Pavia, s. 5, 16: 210-130.

Biondi E., 2000 - Syntaxonomy of the mediterranean chamaephytic and nanophanerophytic vegetation in Italy. Colloques Phytosociologiques, 27 (1997): 123-145.

Biondi E. \& Blasi C., 2015 - Prodromo della vegetazione italiana. Ministero dell'Ambiente e della Tutela del Territorio e del Mare. $<$ http://www.prodromo-vegetazione-italia.org > (ultima consultazione 19 dicembre 2015). 
Bogliani G., Cova C. \& Polani F., 2003 - La natura tra Nure e Scrivia. Provincia di Pavia. Settore Politiche Agricole, Faunistiche e Naturalistiche, Pavia.

Bona E., 2015 - L'atlante corologico della flora vascolare della Lombardia centro-orientale (BG-BS). In: Biodiversità nell'anno di Expo: la straordinaria flora lombarda. Atti della giornata di approfondimento delle conoscenze floristiche. Milano, 23 maggio 2015. Galasso G. \& Mangili F. (eds.). Natura, 105 (2): 77-82.

Briosi G., 1914 - La Stazione di Botanica Crittogamica (Laboratorio Crittogamico Italiano) in Pavia dalla sua fondazione (1871) sino all'anno 1910. - Rapporto chiesto per l'esposizione di Bruxelles 1910 da S. E. il Ministro d'Agricoltura, Industria e Commerico. Atti dell'Istituto Botanico dell'Università di Pavia, s. 2, 13: 412440.

Brusa G. \& Rovelli P., 2010 - Atlante della flora del Parco Agricolo Sud Milano. Provincia di Milano, Parco Agricolo Sud Milano, Milano.

Camacho Villa T.C., Maxted N., Scholten M. \& Ford-Lloyd B., 2005 Defining and identifying crop landraces. Plant Genetic Resources, 3 (3): 373-384. doi: 10.1079/PGR200591

Camera di Commercio Pavia, 2009 - Buono a sapersi. Una provincia da gustare. Pavia. Sagep Editori, Genova.

Carrega M. \& Silla D., 1995 - Ricerche floristiche nel Novese e nel Tortonese (Provincia di Alessandria, Piemonte sud orientale). Parte I. Lycopodiaceae-Araliaceae. Rivista piemontese di storia naturale, 16: $17-76$

Carrega M. \& Silla D., 1996 - Ricerche floristiche nel Novese e nel Tortonese (Provincia di Alessandria, Piemonte sud orientale). Parte II. Umbelliferae-Orchidaceae. Rivista piemontese di storia naturale, 17: 77-149.

Carrega M. \& Silla D., 1999 - Ricerche floristiche nel Novese e nel Tortonese (Provincia di Alessandria, Piemonte sud orientale). (Aggiornamento anni 1994-1997). Rivista piemontese di storia naturale, 20: 3-18.

Casale F., Brambilla M., Falco R. \& Bogliani G., 2011 - Atlante delle Zone di Protezione Speciale della Lombardia. Fondazione Lombardia per l'Ambiente e Regione Lombardia, Milano.

Cavagna P., Camerini G., Fibiani M., Andreani L., Cella R., Concia L. \& Lo Scalzo R., 2012 - Characterization of the rescued 'Voghera' sweet pepper landrace grown in northern Italy. Spanish Journal of Agricultural Research, 10 (4): 1059-1069. doi: 10.5424/ sjar/2012104-2939

Cecchi L. \& Selvi F., 2015a - Heliotropiaceae. Versione 1.0., pubblicata online il 25.02.2015. In: Flora Critica d'Italia. Peruzzi L., Cecchi L., Cristofolini G., Domina F., Greuter W., Nardi E., Raimondo F.M., Selvi F. \& Troìa A. (eds.). Fondazione per la Flora Italiana, Firenze.

Cecchi L. \& Selvi F., 2015b - Hydrophyllaceae. Versione 1.0., pubblicata online il 25.02.2015. In: Flora Critica d'Italia. Peruzzi L., Cecchi L., Cristofolini G., Domina F., Greuter W., Nardi E., Raimondo F.M., Selvi F. \& Troìa A. (eds.). Fondazione per la Flora Italiana, Firenze.

Celesti-Grapow L., Pretto F., Brundu G., Carli E. \& Blasi C. (eds.), 2009 - A thematic contribution to the National Biodiversity Strategy. Plant invasion in Italy, an overview. Ministry for the Environment Land and Sea Protection, Nature Protection Directorate, Roma. + CD-ROM

Celesti-Grapow L., Pretto F., Carli E. \& Blasi C. (eds.)., 2010 - Flora vascolare alloctona e invasiva delle regioni d'Italia. Casa Editrice Università La Sapienza, Roma.

Cesati V., 1835a - Gita agli Apennini. Archivio di Stato di Vercelli, Fondo Arborio Mella, mazzo 145 (manoscritto inedito).

Cesati V., 1835b - Catalogus plantarum quas in Appenninis Bobbiensibus, Castidii, et per Vallis Stafora et Coppus observavi a die 11 ad 22 junii 1835. Archivio di Stato di Vercelli, Fondo Arborio Mella, mazzo 145 (manoscritto inedito).

Cesati V., 1835c - Raccolte negli Apennini. Archivio di Stato di Vercelli, Fondo Arborio Mella, mazzo 145 (manoscritto inedito)

Cesati V., 1844 - Flora. In: Notizie naturali e civili su la Lombardia. Cattaneo C. (ed.). G. Bernardoni di Giovanni, Milano, 1: 259-326.

Christenhusz M.J.M., Reveal J.L., Farjon A., Gardner M.F., Mill R.R. \& Chase M.W., 2011a-A new classification and linear sequence of extant gymnosperms. Phytotaxa, 19: 55-70.
Christenhusz M.J.M., Zhang X.-C. \& Schneider H., 2011b - A linear sequence of extant families and genera of lycophytes and ferns. Phytotaxa, 19: 7-54. doi: 10.11646/phytotaxa.19.1.2

Ciferri R., 1951 - Notizia dell'istituzione di un Erbario Lombardo presso 1'Istituto Botanico dell'Università di Pavia. Nuovo Giornale Botanico Italiano, n. S., 58: 181

Ciferri R., 1961 - Dal lettorato dei semplici all'Istituto ed Orto botanico ed istituti annessi. In: Discipline e maestri dell'Ateneo pavese: Università di Pavia, 1361-1961. AA.VV. (eds.). A. Mondadori, Milano: 153-164.

Ciferri R., 1964 - Gino Pollacci (1872-1963). Atti dell'Istituto Botanico e del Laboratorio Crittogamico dell'Università di Pavia, s. 5, 21 : 25-38

Cole T.C.H., Hilger H.H. \& Selvi F., 2016 - Filogenesi delle Angiosperme - Sistematica delle piante a fiore (Italian translation of: Angiosperm Phylogeny Poster - Flowering Plant Systematics). doi: 10.13140/RG.2.1.1051.0163

Colla L., 1833 - Elogio storico dell'accademico professore Giovanni Battista Balbis. Memorie della Reale Accademia delle scienze di Torino, 36: XXVII-LIV.

Comune di Nibbiano, 2015 - Diga del Molato. $<$ http://www.comune. nibbiano.pc.it/c033029/zf/index.php/servizi-aggiuntivi/index/index/idtesto/92> (ultima consultazione 30 novembre 2015).

Contardi G., 2015 - Stima provinciale delle superfici e rese delle principali colture. Anno 2015. Provincia di Pavia, Settore Agroambientale. $<\mathrm{http}: / / \mathrm{www}$.provincia.pv.it $>$ (ultima consultazione 12 dicembre 2015).

Conti F., Abbate G., Alessandrini A. \& Blasi C. (eds.), 2005 - An annotated checklist of the Italian vascular flora. Palombi Editori, Roma.

Conti F., Alessandrini A., Bacchetta G., Banfi E., Barberis G., Bartolucci F., Bernardo L., Bonacquisti S., Bouvet D., Bovio M., Brusa G., Del Guacchio E., Foggi B., Frattini S., Galasso G., Gallo L., Gangale C., Gottschlich G., Grünanger P., Gubellini L., Iiriti G., Lucarini D., Marchetti D., Moraldo B., Peruzzi L., Poldini L., Prosser F., Raffaelli M., Santangelo A., Scassellati E., Scortegagna S., Selvi F., Soldano A., Tinti D., Ubaldi D., Uzunov D. \& Vidali M., 2007 - Integrazioni alla checklist della flora vascolare italiana. $\mathrm{Na}$ tura Vicentina, 10 (2006): 5-74.

Cristofolini G., 1998 - Qualche nota sulla diversità floristica, sulla biodiversità in generale, e sui modi per misurarla. Informatore Botanico Italiano, 30 (1-3): 7-10

ERSAF, 2010 - Uso del suolo in Regione Lombardia. Atlante descrittivo. Regione Lombardia.

Euro+Med, 2006 onwards - Euro+Med Plantbase - the information resource for Euro-Mediterranean plant diversity. <http://ww2.bgbm. org/EuroPlusMed/> (ultima consultazione 15 novembre 2015).

European Commission, 2006 - Natura 2000 in the Continental region. Office for Official Publications of the European Communities, Luxembourg.

Farneti R., 1900 - Aggiunte alla flora pavese e ricerche sulla sua origine. Atti dell'Istituto Botanico dell'Università di Pavia, s. 2, 6 123-164.

Fattorini S., 2015 - On the concept of chorotype. Journal of Biogeography, 42 (11): 2246-2251. doi: 10.1111/jbi.12589

Filipello S. (ed.), 1978 - Carta delle conoscenze floristiche d'Italia. Informatore Botanico Italiano, 9 (3): 281-284.

Galasso G., 2015a - Conoscenze floristiche in Lombardia: stato dell'arte. In: Biodiversità nell'anno di Expo: la straordinaria flora lombarda. Atti della giornata di approfondimento delle conoscenze floristiche. Milano, 23 maggio 2015. Galasso G. \& Mangili F. (eds.). Natura, 105 (2): 31-38.

Galasso G., 2015b - Appendice. Elenco degli endemiti lombardi. In: Biodiversità nell'anno di Expo: la straordinaria flora lombarda. Atti della giornata di approfondimento delle conoscenze floristiche. Milano, 23 maggio 2015. Galasso G. \& Mangili F. (eds.). Natura, 105 (2): 31-38.

Garovaglio S., 1862 - Sulle attuali condizioni dell'Orto Botanico della Università di Pavia. Ditta Tipografica Eredi Bizzoni, Pavia.

Gentile S., 1975 - Ricerche sui faggeti dell'Appennino ligure. Notiziario della Società Italiana di Fitosociologia, 9 (1974): 131138 
Gentile S. \& Sartori F., 1975 - La vegetazione dei calanchi nei terreni eo-miocenici delle valli Staffora e Curone. Atti dell'Istituto Botanico e del Laboratorio Crittogamico dell'Università di Pavia, s. 6, 10: 59-115.

Giacomini V., 1952 - Commemorazione del Dott. Luigi Ceroni. Nuovo Giornale Botanico Italiano, n. s., 59 (2): 166-167.

Giacomini V., 1981 - Ciferri, Raffaele. Dizionario Biografico degli Italiani, 25. Istituto Treccani, [Roma]. <http://www.treccani.it/enciclopedia/raffaele-ciferri_(Dizionario_Biografico) $>$ (ultima consultazione 24 dicembre 2015).

Giacomini V. \& Fenaroli L., 1958 - La Flora. In: Conosci l'Italia. Volume II. Touring Club Italiano, Milano.

Giordana F., 2010a - Flora Cremonese: cartine di distribuzione, 15 apr 2010. <http://flora.garz.net/ giordana/FLORA-CR.TXT> (ultima consultazione 2 agosto 2016).

Giordana F., 2010b - Flora Lodigiana: cartine di distribuzione, 15 apr 2010. <http://flora.garz.net/ giordana/FLORA-LO.TXT> (ultima consultazione 14 ottobre 2015).

Giulietti C., 1900 - Notizie naturali geologiche di Casteggio. Tipografia Enrico Sparolazzi, Casteggio.

Gobbi C., 2004 - Il radar che veglia sul nord Italia. La Provincia Pavese, 25 aprile 2004: 33.

Govi G., 1995 - Farneti, Rodolfo. Dizionario Biografico degli Italiani, 45. Istituto Treccani, [Roma]. <http://www.treccani.it/enciclopedia/rodolfo-farneti_(Dizionario_Biografico)> (ultima consultazione 24 dicembre $20 \overline{15}$ ).

Guzzi A., 2004 - Il sistema agro-forestale dell'Oltrepò pavese. Oltrepò pavese: suolo, natura e acqua, 13-14 ottobre 2004, Salice Terme [Godiasco Salice Terme]. <http://ita.arpalombardia.it> (ultima consultazione 16 dicembre 2015).

Harpke D., Kerndorff H., Pasche E. \& Peruzzi L., 2016 - Neotypification of the name Crocus biflorus Mill. (Iridaceae) and its consequences in the taxonomy of the genus. Phytotaxa, 260 (2): 131143. doi: $10.11646 /$ phytotaxa.260.2.3

Hernández-Ledesma P., Berendsohn W.G., Borsch T., Von Mering S., Akhani H., Arias S., Castañeda-Noa I., Eggli U., Eriksson R., Flores-Olvera H., Fuentes-Bazán S., Kadereit G., Klak C., Korotkova N., Nyffeler R., Ocampo G., Ochoterena H., Oxelman B., Rabeler R.K., Sanchez A., Schlumpberger B.O. \& Uotila P., 2015 A taxonomic backbone for the global synthesis of species diversity in the angiosperm order Caryophyllales. Willdenowia, 45(3): 281383. doi: $10.3372 /$ wi.45.45301

Källersjö M., Bergqvist G. \& Anderberg A.A., 2000 - Generic realignment in primuloid families of the Ericales s.l.: a phylogenetic analysis based on DNA sequences from three chloroplast genes and morphology. American Journal of Botany, 87 (9): 1325-1341.

Macchi P., 2005 - La flora della provincia di Varese. Provincia di Varese Edizioni, Varese.

Maffei L., 1921 - Aggiunte alla flora Pavese. Atti dell'Istituto Botanico dell'Università di Pavia, s. 2, 18: 137-150.

Maffi L., 2010 - Storia di un territorio rurale. Vigne e vini nell'Oltrepò Pavese. FrancoAngeli, Milano.

Martini F., Bona E., Federici G., Fenaroli F. \& Perico G., 2012 - Flora vascolare della Lombardia centro-orientale, 1: Parte generale. LINT, Trieste.

Massi E., 1967 - L'Oltrepò pavese: i fattori naturali, il fattore umano. C.A.S., Roma.

Medici G., 1932 - Monografia economico-agraria dell'Oltrepò pavese. Tipografia Cooperativa, Pavia.

Merlo M., 1979 - Leggende lombarde. Longanesi \& C., Milano.

Moretti G., 1822 - Flora veronensis quam in prodromum Florae Italiae septentrionalis exhibet Cyrus Pollinius. Vol. primo in $8 .^{\circ}$ di pagine 535 , oltre alla prefazione che è di pag XXXV, con due tavole in rame. Verona 1822, dalla Società tipografica. Biblioteca Italiana ossia Giornale di Letteratura, Scienze ed Arti, 27 (1): 364-376.

Moretti G., 1851 - Sull'Apios tuberosa. Lettera del prof. Moretti al sig. ingegnere Dossena. Giornale Agrario Lombardo-Veneto e continuazione degli Annali Universali di Tecnologia applicati all'Agricoltura, s. 3, 5 (2): 123-130.

Nocca D. \& Balbis G.B., 1816 - Flora ticinensis. Tipographia J.J. Capelli, Ticini, 1.
Nocca D. \& Balbis G.B., 1821 - Flora ticinensis. Tipographia J.J. Capelli, Ticini, 2.

Ottone C. \& Rossetti R., 1980 - Condizioni termo-pluviometriche della Lombardia. Atti dell'Istituto Geologico dell'Università di Pavia, 29: $27-48$.

Pavan Arcidiaco L., Peccenini Gardini S. \& Terzo V., 1982 - Notizie sugli erbari del ginnasio-liceo «U. Foscolo» di Pavia. Bollettino della Società Pavese di Storia Patria, n. s., 34: 254-258.

Pavarino G.L., 1914 - Intorno alla flora del calcare e del serpentino nell'Appennino bobbiese. Contribuzione seconda. Atti dell'Istituto Botanico dell'Università di Pavia, s. 2, 14: 19-42.

Pavarino G.L., 1915 - Intorno alla flora del calcare e del serpentino nell'Appennino Bobbiese. Contribuzione prima. Atti dell'Istituto Botanico dell'Università di Pavia, s. 2, 12: 21-56

Pavesi V., 1906 - Elenco di piante dell'alto Apennino Pavese. Atti della Società Italiana di Scienze Naturali e del Museo Civico di Storia Naturale di Milano, 45: 45-54.

Perracino M., 2010 - Atlante dei SIC della Provincia di Pavia. Regione Lombardia e Fondazione Lombardia per l'Ambiente, Milano.

Peruzzi L., Conti F. \& Bartolucci F., 2014 - An inventory of vascular plants endemic to Italy. Phytotaxa, 168 (1): 1-75. doi: 10.11646/ phytotaxa.168.1.1

Peruzzi L., Conti F. \& Bartolucci F., 2014 onwards - Italian vascular plant endemics - linked to Phytotaxa 168: 1-75 (2014) and continuously updated. $<$ https://docs.google.com/spreadsheet/ ccc?key=0A iJpWnsqul0MdG1hSkk1 azBjN3BoLXB4VTAzc311 Smc\#gid=0> (ultima consultazione 23 novembre 2015).

Peruzzi L., Domina G., Bartolucci F., Galasso G., Peccenini S., Raimondo F.M., Albano A., Alessandrini A., Banfi E., Barberis G., Bernardo L., Bovio M., Brullo S., Brundu G., Brunu A., Camarda I., Carta L., Conti F., Croce A., Iamonico D., Iberite M., Iiriti G., Longo D., Marsili S., Medagli P., Pistarino A., Salmeri C., Santangelo A., Scassellati E., Selvi F., Soldano A., Stinca A., Villani M., Wagensommer R.P. \& Passalacqua N.G., 2015 - An inventory of the names of vascular plants endemic to Italy, their loci classici and types. Phytotaxa, 196 (1): 1-217. doi: 10.11646/ phytotaxa.196.1.1

Piazza C., 2014 - Çedron, Çedar (Cedro, Zucca da mostarda). In: Frutta e buoi... Quaderno della biodiversità agricola parmense. AA.VV. (eds.). Servizio Agricoltura e Risorse Naturali, Provincia di Parma, Parma: 169-170.

Piazza R., 2015 - La ricerca floristica in Valtellina e Valchiavenna. In: Biodiversità nell'anno di Expo: la straordinaria flora lombarda. Atti della giornata di approfondimento delle conoscenze floristiche. Milano, 23 maggio 2015. Galasso G. \& Mangili F. (eds.). Natura, 105 (2): 97-106.

Pignatti S., 1957 - La vegetazione messicola delle colture di Frumento, Segale e Avena nella provincia di Pavia. Atti dell'Istituto Botanico e del Laboratorio Crittogamico dell'Università di Pavia, s. 5, 12: 243-319.

Pignatti S., 1967 - Über die submediterrane Vegetation des pavesischen Appennins. Mitteilungen der Ostalpin-Dinarischen Pflanzensoziologischen Arbeitsgemeinschaft, 7: 143-148.

Pignatti S., 1982 - Flora d'Italia, 1-3. Edagricole, Bologna.

Pinna M., 1970 - Contributo alla classificazione del clima d'Italia. Rivista Geografica Italiana, 77 (2): 129-152.

Pirola A., 1964 - Alcune novità per la flora pavese. Atti dell'Istituto Botanico e del Laboratorio Crittogamico dell'Università di Pavia, s. 5, 21: 39-43.

Pirola A., 1992 - L'Orto Botanico dell'Università di Pavia. In: Orti Botanici, Giardini Alpini, Arboreti Italiani. Raimondo F.M. (ed.). Edizioni Grifo, Palermo.

Pollacci G., 1918 - Aggiunte alla flora ticinese. Atti dell'Istituto Botanico dell'Università di Pavia, s. 2, 15 (1918): 53-62.

Pollacci G., 1936 - Domenico Nocca. Atti dell'Istituto Botanico e del Laboratorio Crittogamico dell'Università di Pavia, s. 4, 8: III-V.

Regione Lombardia, 2002-2015 - LombardiaBeniCulturali. <http:// www.lombardiabeniculturali.it/> (ultima consultazione 21 dicembre 2015).

Rinaldi G., 2009 - Lorenzo Rota 150 anni dopo. Orto Botanico di Bergamo "Lorenzo Rota”, Bergamo. 
Rivas-Martínez S., Penas A. \& Díaz T.E., 2004 - Biogeographic Map of Europe. Cartographic Service, University of León, León. <http:// www.globalbioclimatics.org > (ultima consultazione 8 dicembre 2015).

Rivas-Martínez S., Rivas-Sáenz S., Penas A. \& Díaz T.E., 2015a Computerized Bioclimatic Maps of the World: Bioclimates of Europe and Mideast. Centro de Investigaciones Fitosociólogicas, Madrid. <http://www.globalbioclimatics.org > (ultima consultazione 8 dicembre 2015).

Rivas-Martínez S., Rivas-Sáenz S., Penas A. \& Díaz T.E., 2015b Computerized Bioclimatic Maps of the World: Continentality of Europe and Mideast. Centro de Investigaciones Fitosociólogicas, Madrid. <http://www.globalbioclimatics.org > (ultima consultazione 8 dicembre 2015).

Rivas-Martínez S., Rivas-Sáenz S., Penas A. \& Díaz T.E., 2015c Computerized Bioclimatic Maps of the World: Ombrotypes of Europe and Mideast. Centro de Investigaciones Fitosociólogicas, Madrid. <http://www.globalbioclimatics.org> (ultima consultazione 8 dicembre 2015).

Rivas-Martínez S., Rivas-Sáenz S., Penas A. \& Díaz T.E., 2015d Computerized Bioclimatic Maps of the World: Thermotypes of Europe and Mideast. Centro de Investigaciones Fitosociólogicas, Madrid. <http://www.globalbioclimatics.org > (ultima consultazione 8 dicembre 2015).

Romani E. \& Alessandrini A., 2001 - Flora Piacentina. Compendio del patrimonio floristico della provincia di Piacenza (EmiliaRomagna). Museo Civico di Storia Naturale di Piacenza, Piacenza.

Rossetti R. \& Ottone C., 1979 - Esame preliminare delle condizioni pluviometriche dell'Oltrepò Pavese e dei valori critici delle precipitazioni in relazione ai fenomeni di dissesto franoso. Geologia Applicata e Idrogeologia, 14 (3): 83-99.

Rossi G. \& Ardenghi N.M.G., 2015 - Erbario dell'Università di Pavia (PAV). <http://sciter.unipv.eu/site/home/risorse/sezione-di-ecologia-del-territorio/articolo720004775.html> (ultima consultazione 27 dicembre 2015).

Rossi G., Parolo G., Galasso G., Assini S., Sartori F., Pirola A., Bracco F., Frattini S., Banfi E., Bona E., Perico G. \& Giordana F., 2005 Stato attuale e progressi delle conoscenze floristiche in Lombardia dal 1978 ad oggi. In: Stato delle Conoscenze sulla Flora Vascolare d'Italia. Scoppola A. \& Blasi C. (eds.). Palombi Editori, Roma: 103-107 + cartografia.

Rossi G., Montagnani C., Gargano D., Peruzzi L., Abeli T., Ravera S., Cogoni A., Fenu G., Magrini S., Gennai M., Foggi B., Wagensommer R.P., Venturella G., Blasi C., Raimondo F.M. \& Orsenigo S. (eds.), 2013 - Lista Rossa della Flora Italiana. 1. Policy species e altre specie minacciate. Comitato Italiano IUCN e Ministero dell'Ambiente e della Tutela del Territorio e del Mare, Roma. <http://www.governo.it/backoffice/allegati/71184-8693.pdf>

Rota L., 1847 - Prospetto delle Piante fanerogame finora ritrovate nella Provincia Pavese. Giornale Botanico Italiano, 2 (1, 2): 73-82 + 247-292.

Rothfels C.J., Sundue M.A., Kuo L.-Y., Larsson A., Kato M., Schuettpelz E. \& Pryer K.M., 2012 - A revised family-level classification for eupolypod II ferns (Polypodiidae: Polypodiales). Taxon, 61 (3): 515-533.

Saccardo P.A., 1895 - La botanica in Italia. Materiali per la storia di questa scienza. Tipografia Carlo Ferrari, Venezia.

Saccardo P.A., 1901 - La botanica in Italia. Materiali per la storia di questa scienza. Parte seconda. Tipografia di Carlo Ferrari, Venezia.

Scagni U., 2003 - La Resistenza scolpita nella pietra. Edizioni Guardamagna, Varzi.

Scagni U., 2009 - Fascismo e antifascismo in provincia di Pavia. Edizioni Guardamagna, Varzi.

Sircana G., 2012 - Montemartini, Gabriele Luigi. Dizionario Biografico degli Italiani, 76. Istituto Treccani, [Roma]. <http://www.treccani. it/enciclopedia/gabriele-luigi-montemartini_(Dizionario_Biografico) $>$ (ultima consultazione 24 dicembre 2015).

Sistema Museale di Ateneo, 2015 - Il Museo Civico. <http://musei. unipv.it/storianat/storia/civico.html $>$ (ultima consultazione $26 \mathrm{di}$ cembre 2015).
Soldano A., 1980 - Naturalizzazione nel pavese di Amaranthus bouchonii Thell. e di altre sette esotiche nuove per la Lombardia. Considerazioni distributive su altre specie già note. Atti dell'Istituto Botanico e del Laboratorio Crittogamico dell'Università di Pavia, s. 6, 13 (1978-1979): 137-143.

Soldano A., 1983 - L'attività giovanile di Vincenzo Cesati. Atti dell'Istituto Botanico e del Laboratorio Crittogamico dell'Università di Pavia, s. 7, 2: 65-94.

Soó R. von, 1926 - Systematische Monographie der Gattung Melampyrum. Repertorium Specierum Novarum Regni Vegetabilis, 23 (4-11): 159-176.

Taramelli T., 1916 - Descrizione geologica della Provincia di Pavia. Seconda edizione notevolmente aumentata. Istituto Geografico De Agostini, Novara.

The Angiosperm Phylogeny Group, 2016 - An update of the Angiosperm Phylogeny Group classification for the orders and families of flowering plants: APG IV. Botanical Journal of the Linnean Society: 1-20. doi: 10.1111/boj.12385

Thiers B., 2014 onwards - Index herbariorum: a global directory of public herbaria and associated staff. New York Botanical Garden. $<$ http://sweetgum.nybg.org/science/ih/> (ultima consultazione 29 dicembre 2015).

Tison J.-M., Jeanmonod D. \& Molina J., 2004 - Tragopogon eriospermus Ten. In: Notes et contributions à la flore de Corse, XX. Jeanmonod D. \& Schlüssel A. (eds.). Candollea, 59 (1): 74-76.

Truzzi A., 2015 - Stato delle conoscenze sulla flora vascolare del mantovano. In: Biodiversità nell'anno di Expo: la straordinaria flora lombarda. Atti della giornata di approfondimento delle conoscenze floristiche. Milano, 23 maggio 2015. Galasso G. \& Mangili F. (eds.). Natura, 105 (2): 57-62.

Ubaldi D., Zanotti A.L., Mondino G.P., Troger J.V. \& Puppi G., 1993 Contributo alla conoscenza degli ostrieti e dei querceti caducifogli dell'Appennino Piacentino e Ligure. Annali di Botanica (Roma), 51 (10), suppl. 10: 28-45.

Università degli Studi di Pavia, 2005-2012 - I professori dell'Università di Pavia (1859-1961). <http://prosopografia.unipv.it> (ultimo accesso il 24 dicembre 2015).

Università di Torino, Orto botanico, 1929 - Studi sulla vegetazione nel Piemonte pubblicati a ricordo del II centenario della fondazione dell'Orto botanico della R. Università di Torino, 1729-1929. Luigi Checchini, Torino.

Viola S., 1952 - Naturalizzazione e distribuzione della Allionia nyctaginea nel Pavese. Nuovo Giornale Botanico Italiano, n. s., 58 (1951; 3-4): 490-491.

Viola S., 1953 - Nuove stazioni di piante rare, medicinali o avventizie. Nuovo Giornale Botanico Italiano, n. s., 59 (1952; 2-4): 503-505.

Visconti A., 2012a - La fondazione dell'Orto botanico di Brera e gli anni della direzione dell'abate vallombrosano Fulgenzio Vitman (1728-1806) tra assolutismo asburgico ed età napoleonica Atti della Società Italiana di Scienze Naturali e del Museo Civico di Storia Naturale di Milano, 153(I): 27-48.

Visconti A., 2012b - Gli Orti di Pavia e di Milano nel periodo dell'Assolutismo asburgico. La Giornata di Studi di Orticola di Lombardia 2012. <http://www.orticola.org/orticola/?page_id=4787> (ultimo accesso il 30 novembre 2015).

Visconti A., 2015 - Il botanico Fulgenzio Vitman e i rapporti fra l'Università di Pavia e le Scuole Palatine di Milano. In: Almum Studium Papiense. Storia dell'Università di Pavia. Volume 2: Dall'età austriaca alla nuova Italia. Tomo I: L'età austriaca e napoleonica. Mantovani D. (ed.). Cisalpino, Milano: 181-182.

Volk O.H., 1958 - Trockenrasen aus der Umgebung von Pavia. Atti dell'Istituto Botanico e del Laboratorio Crittogamico dell'Università di Pavia, s. 5, 16: 17-35. 